\title{
Essays on the interaction between risk and market structure in electricity markets
}

\author{
by \\ Gabriel Godofredo Fiuza de Braganca
}

\author{
A thesis \\ submitted to the Victoria University of Wellington \\ in fulfilment of the \\ requirements for the degree of \\ Doctor of Philosophy \\ in Economics. \\ Victoria University of Wellington
}

2011 



\begin{abstract}
This thesis proposes a new framework to jointly analyze electricity spot market and hedging decisions in an oligopolistic setup. Firstly, we find that, when exogenous, both quantity of electricity hedged by contract and vertical integration decrease the equilibrium spot price. Secondly, we use a hybrid approach and show that market structure can affect a generator's decision to vertically integrate under uncertain demand. Thirdly, we consider uncertainty in costs and demand and show that concentration in the spot market, for a given hedge quantum, can increase forward prices and affect the slope of the forward curve. Our empirical results indicate that the model fits the New Zealand electricity market well. This evidence that market structure and hedging decisions are closely connected is further explored in a three period equilibrium model for the spot and forward markets, where hedging occurs prior to the submission of supply curves. Taking into account demand-side and supply-side uncertainties, we find that when hedging is endogenous, hedging quantities are affected by spot market parameters, but market power is itself mitigated in the conscious hedging choice of generators. We also show that forward markets can coexist with highly vertically integrated markets. The importance of our results is general. Our models can be used by policy makers to analyze investment and forward price implications of changes in the spot market structure. Our results also indicate that electricity generators, in equilibrium, face a trade-off between market power and hedging. Given that it is socially beneficial to manage risk, the equilibrium impact of their choices on welfare should not be considered in isolation by competition authorities.
\end{abstract}




\section{Acknowledgments}

Firstly, I would like to thank my supervisors, Prof. Lew Evans and Dr. Toby Daglish, for the support and guidance they have provided throughout my PhD program. I have been extremely lucky to have supervisors who cared so much about my research, and who responded to my questions and queries so promptly. I am also grateful to Prof. Glenn Boyle for endorsing my admission to the $\mathrm{PhD}$ program and for supporting me extensively during my first year. Secondly, I am thankful to the Brazilian Institute of Applied Economic Research (IPEA), specially to Dr. Ronaldo Seroa da Motta, for the encouragement to undertake this challenging and life changing project. Thirdly, I would like to thank Ms. Bronwyn Howell and Dr. Suzi Kerr for giving me valuable opportunities to present and discuss my work with distinguished academics and professionals. Fourthly, I would like to thank the staff of both the School of Economics and Finance (SEF) and the New Zealand Institute for the Study of Competition and Regulation (ISCR) for enabling my participation in insightful seminars/congresses and, particularly, Ms. Suzanne Freear, Ms. Amanda Bristol and Ms. Kathy McKenzie, for all the administrative assistance. Also, I would like to record my acknowledgement and gratitude for the financial support from the Victoria University of Wellington, ISCR and Motu Economic and Public Policy Research. Lastly, I wish to express my love and gratefulness to my beloved wife, Roberta, for her understanding and endless love, through the duration of my studies. This Thesis is dedicated to God. 


\section{Contents}

1 Introduction 1

1.1 Electricity markets and idiosyncrasies $\ldots \ldots \ldots \ldots . \ldots$

1.2 Price behavior and determinants $\ldots \ldots \ldots \ldots \ldots$

1.3 Thesis outline . . . . . . . . . . . . . . . 10

2 Basic model $\quad 15$

2.1 Model set-up and definitions $\ldots \ldots \ldots \ldots$

2.2 Equilibrium results . . . . . . . . . . . . 20

3 Vertical integration $\quad 29$

3.1 Motivation . . . . . . . . . . . . . . . . . . . . . . . . . . . . . 29

3.2 General assumptions . . . . . . . . . . . . . . . 31

3.3 Model 1: uncertain rivals' contracts . . . . . . . . . . . . 34

3.4 Model 2: uncertain short-term demand . . . . . . . . . . . 39

4 Concentration and forward prices 43

4.1 Literature and motivation . . . . . . . . . . . . . . 43

4.2 Model and Results . . . . . . . . . . . . . . . . . . 45

4.3 Empirical Exercise . . . . . . . . . . . . . . . . . 52

4.3 .1 First step . . . . . . . . . . . . . . . 55

4.3 .2 Second step $\ldots \ldots \ldots \ldots \ldots$

4.4 Summary . . . . . . . . . . . . . . . 63 
5 Investing in vertical integration $\quad 65$

5.1 Motivation . . . . . . . . . . . . . . . 65

5.2 Model . . . . . . . . . . . . . . . . . . 68

5.3 Numerical Example . . . . . . . . . . . . . . . . 74

6 Market power and hedging decision $\quad 85$

6.1 The role of preferences . . . . . . . . . . . . . . . 88

6.2 Basic assumptions and spot market results . . . . . . . . 90

6.3 Hedging decision and equilibrium results . . . . . . . . . 96

6.4 Numerical exercise . . . . . . . . . . . . . . . . . . . . . . 101

6.4.1 Calibration Methodology . . . . . . . . . . . . . 102

6.4.2 Illustrative example: New Zealand . . . . . . . . . . . 104

6.4.3 The Role of Cost and Demand Volatilities . . . . . . . 106

6.5 Vertical integration . . . . . . . . . . . . . . . 110

6.5 .1 Numerical results . . . . . . . . . . . . . . . 112

$\begin{array}{lll}7 & \text { Conclusion } & 117\end{array}$

$\begin{array}{ll}\text { A Chapter } 2 & 131\end{array}$

A.1 Proof of lemma $2.2 .1 \ldots \ldots$. . . . . . . . . . . . 131

A.2 Proof of lemma $2.2 .2 \ldots \ldots \ldots$

A.3 Proof of proposition 2.2.3 . . . . . . . . . . . . 134

$\begin{array}{ll}\text { B Chapter } 3 & 137\end{array}$

B.1 Proof of lemma 3.3.1 . . . . . . . . . . . . . 137

B.2 Proof of lemma $3.3 .2 \ldots \ldots \ldots$. . . . . . . . . . . 138

B.3 Proof of proposition 3.3.3 . . . . . . . . . . . . . . 140

B.4 Proof of proposition 3.4.1 . . . . . . . . . . . . 142

B.5 Proof of proposition 3.4.2 . . . . . . . . . . . . 146

$\begin{array}{lr}\text { C Chapters } 4 \text { and } 5 & 149\end{array}$

C.1 Proof of corollary $4.2 .2 \ldots \ldots$. . . . . . . . . . . . 149

C.2 Proof of lemma $5.2 .1 \ldots \ldots$. . . . . . . . . . 150 
C.3 Proof of lemma $5.2 .2 \ldots \ldots$. . . . . . . . . . 156

C.4 Proof of proposition $5.2 .3 \ldots \ldots \ldots$. . . . . . . . 157

$\begin{array}{ll}\text { D Chapter } 6 & 161\end{array}$

D.1 Proof of proposition $6.3 .1 \ldots \ldots \ldots \ldots 16 \ldots \ldots$

D.2 Proof of proposition $6.3 .2 \ldots \ldots \ldots \ldots$

D.3 Proof of proposition 6.3.3 . . . . . . . . . . . . . . 164 


\section{Chapter 1}

\section{Introduction}

The objective of this thesis is to analyze how risk and market structure affect prices and economic decisions in decentralized electricity markets. Electricity has unique characteristics that distinguish it from other commodities. Classical assumptions from the financial literature do not apply to electricity derivatives and hedging markets. In addition, electricity markets frequently present market power issues and intricate structure. There is a large body of literature addressing each of these matters individually, but very few articles address the interaction between finance and market power in electricity markets. Our main contribution consists in developing a formal framework to analyze, under realistic assumptions, forward pricing, vertical integration and hedging decisions in electricity markets. In particular, We assume uncertainty, oligopolistic behavior and uniformprice auctions in our models.

\subsection{Electricity markets and idiosyncrasies}

Electricity is an important and ubiquitous good. Modern economies have experienced rapid growth in the consumption of electricity, either due to the expansion of production or because of the increasing use of electrical and electronic devices. Consumers and firms demand large continuous 
supplies of electricity at all hours of the day in most countries. For example, according to Birol (2009), the world consumption of electricity increased from 6,799 TWh in 1980 to 16,429 TWh in 2007 and is expected to achieve 28,930 TWh in 2030.

The power industry is roughly described by the existence of three sectors, each with very particular characteristics. The generation sector is composed of power plants and produces electricity from particular fuels or renewable sources. The transmission sector is responsible for transporting electricity from the plants to large consumers and/or populated regions. The distribution/retail sector is responsible for delivering/selling electricity to individual households and commercial customers. These sectors are interconnected by a network/grid that crucially depends on its geographical, energetic and economic characteristics. Aspects such as the location of power plants and consumers, characteristics of climate and terrain, and demographic characteristics affect the economic performance of the grid.

The uniqueness of electricity markets is explained above all by the product's unusual characteristics. First, at the current state of technology, electricity is economically non-storable. Further, it is transported from generation plants to final consumers instantly. This means that not only must electricity supply match consumption continuously but also that ongoing delivery requires ongoing generation. As pointed out by Meade and Evans (2006), because of its physical characteristics, electricity presents a rather unusual network. Unlike other network industries such as gas, sewage or railroads, electricity networks do not involve the physical delivery of a given product to/from specified production/delivery points. This raises a second electricity demand-side issue. According to Stoft (2002), the second demand-side issue is the impossibility of controlling real-time flow of power to specific customers.

As posed by Brennan (2003), in addition to being an important commodity, electricity is both vulnerable to supply-demand imbalances (be- 
cause storage costs are prohibitive) and interconnection (because switching costs are prohibitive). The electricity system physics imply that supply is always equal to consumption (including physical losses). Therefore, instantaneous differences between supply and demand can be indicated by neither flows of power (quantities) nor differences expressed by contracts. The balance of a real-time electricity market is in fact signaled by physical parameters such as frequency and/or voltage. Since supply and consumption are permanently balanced, for a given demand, any reduction in supply decreases the voltage and frequency of all the interconnection. ${ }^{1}$ Besides, constraints caused by physical limitations of particular transmission and/or distribution lines have immediate effects over the quality and capacity of the entire system. Consequently, in order to keep the market balanced and the same frequency/voltage in the grid, any binding constraint at any point of the network is almost immediately transmitted to increases in spot prices. For these reasons, if one supplier fails to meet the demands of its customers, not only will those customers lose service, but also all customers sharing the distribution grid will either lose power or face higher prices.

Therefore, quality and reliability are essential issues in the industry. The system operator is a natural monopoly service which is responsible for assuring the reliability of the system and the quality of the power delivered. Its mission consists of keeping the system in balance by maintaining supply equal to demand, keeping the voltage and the frequency at the right levels, and restarting the system when it suffers a complete collapse. As discussed before, any imbalance between supply and demand causes the system frequency/voltage to deviate from the standard, which can potentially harm electricity consumers and producers (e.g. plant damage). In fact, consumers can incur large losses due to problems such as equipment malfunctions and short-circuits caused by these deviations. In extreme

\footnotetext{
${ }^{1} \mathrm{An}$ interconnection is a portion of the power grid connected by alternating current (AC) power lines. The frequency in every utility in an entire interconnection is exactly the same.
} 
cases, occurrences such as brownouts and/or blackouts cause enormous social and economic burden. To sum up, the system operator must guarantee that multiple outputs such as voltage, electrical energy, power and/or frequency are delivered in real time within very narrow bounds.

Different markets may be required to handle larger deviations and emergencies (e.g. unpredicted disruptions of supply). These emergency services are collectively known as ancillary services. They usually benefit the entire market and are either public goods or generate large externalities. Consequently, all of the electricity markets have a heavily regulated demand side. The system operator is also responsible for coordinating these markets and for facilitating demand response for these ancillary services. The operator is usually able to buy energy and frequently has various grades of operating reserves available to balance the system and to provide reliability. The management of reliability requirements determines not only short-term reliability, but the risk inherent to the market and therefore the long-run investment in generation.

The system operator needs to manage the system in real time to keep it physically secure. This is the reason why system operators typically assure the operation of real time spot markets. Unlike in regular markets, electricity customers can take power in real time without a contract. In the limit, they can cause other customers to be blacked out without suffering disruption of their own electricity. The operator needs a small lead time for planning to deal with these externalities. According to Stoft (2002), this lead time is generally accepted to be approximately a day. Beyond that the spot market system has no need to pay attention to the energy market. Notice that electricity industries, with well-functioning competitive spot markets, rely on the system operator to tackle uncertainties and keep the real-time markets balanced and reliable. Medium-term and long-term arrangements for the supply of power are made either in forward markets or in futures markets.

Decentralized electricity industries are characterized by the coexistence 
of several overlapping markets. Electricity trading over any specific period of time can start from years to minutes before the actual delivery. Medium-term and long-term contracts are generally traded through forward and/or futures markets. They are usually financial markets in the sense that the delivery of electricity is optional and the seller's obligation is strictly financial (i.e. contracts are settled in cash). On the other hand, short-term transactions are made in the so called spot markets. In some competitive electricity industries, the definition of spot markets comprises both the day-ahead market and the real-time market. It is true that dayahead markets increase market completeness and potentially raise shortterm liquidity. However, despite their specific features, day-ahead markets are also financial markets. Similarly to forwards and futures, dayahead trading is a straightforward negotiation in the sense that bid offers, quantities sold/delivered and prices are easily established. On the other hand, electricity real-time markets present unique features. First, they are the only physical markets (i.e. involves actual delivery of power). Second, as mentioned, they present issues and externalities that demand a close regulation by the system operator. For the purposes of this thesis, spot markets will be taken to mean the real-time markets exclusively.

Electricity markets are frequently cleared by auctions in order to improve price signals. There is an open debate about what is the best auction design in terms of generating most social/economic welfare. There are two predominant models of auctions in power markets. The uniform-price (or single price) auction, where all suppliers receive the same equilibrium or market clearing price and the pay-as-bid auction, where the price paid to the winners are the same as their actual bids. The advantages and disadvantages of each framework for electricity wholesale markets are still controversial. According to Oren (2004), most economists would agree that the uniform-price auctions are better than pay-as-bid auctions for complete and homogeneous markets. The real dispute concerns incomplete and/or oligopolistic markets. The pay-as-bid could better reduce the price 
volatility in some situations of market incompleteness. It could also avoid the possibility of collusive equilibria present in repeated uniform-price auctions. ${ }^{2}$ Several electricity markets, such as for example California (CAISO), Pennsylvania-New Jersey-Maryland (PJM) ${ }^{3}$, Texas (ERCOT), New York (NYSE) and New Zealand (NZEM), adopt uniform-price auctions.

This dichotomy between uniform-price and pay-as-bid auctions is valid above all for electricity financial markets such as forward, futures and dayahead markets. In terms of our definition of spot markets that corresponds exclusively to real-time markets (e.g. NZEM), uniform-price auction is in fact a more feasible market clearing approach. Since it is not possible to control instantaneous flows of electricity in the network, a pure pay-as-bid auction is very hard to implement in real time: it requires an underestimated/overestimated supply-demand and pricing mechanism. In fact, it is extremely costly in the horizon of a real time market (hours or minutes) to reconcile bids in a pay-as-bid auction to the correspondent flows actually (physically) dispatched/received. For these reasons we assume that spot markets are cleared through uniform-price auctions. We also assume that contract (financial) markets such as forwards/futures or day-ahead are cleared by pay-as-bid auctions. Therefore, in theory, players in our framework are allowed to trade in both auction approaches ${ }^{4}$.

Market structure plays an important role in electricity markets ${ }^{5}$. First, the characteristics of a particular grid determine the cost structure and directly affect firms' marginal costs and their decisions. That is, aspects such

\footnotetext{
${ }^{2}$ See Kahn, Cramton, Porter, and Tabors (2001), Oren (2004) and Cramton and Stoft (2007) for a detailed and accessible discussion of advantages and disadvantages of each approach.

${ }^{3}$ The US states covered by the PJM are all or most of Delaware, District of Columbia, Maryland, New Jersey, Ohio, Pennsylvania, Virginia and West Virginia. Parts of Indiana, Illinois, Kentucky, Michigan, North Carolina and Tennessee.

${ }^{4}$ In fact, the contract market is assumed as exogenous in most chapters of the thesis. Chapter 6 relaxes this hypothesis and analyzes the determinants of hedging decision.

${ }^{5}$ The notion of market structure is closely tied to technology and ownership and was developed as part of the "structure-conduct-performance" paradigm of industrial organization in the early 1950's.
} 
as the number of generators and retailers in each point of the grid, the disposition of transmission (and distribution) power lines and the proportions of sources of power (hydro, thermal, nuclear, wind, etc.) are closely tied to costs and, hence, the market structure of a specific industry. Second, because of the usually large returns to scale involved in the businesses, most electricity wholesale markets are dominated by a small number of firms. This raises concerns about the existence of wholesale market power in most of the electricity industries. In other words, the ability of a relatively large generator to influence equilibrium price outcomes by their supply decisions. This is the rationale for the oligopolistic behavior assumption of our models. Some decentralized electricity markets (e.g NZEM, Spain (IEM) and Germany(GEM)) are also characterized by the existence of firms with large positions in both the generation and the retail markets: generally referred to as vertical integration. Possible causes and consequences of vertical integration is analyzed in subsequent chapters.

\subsection{Price behavior and determinants}

The non-storability of electricity, combined with the strong interdependence between players in the same network, results in the following features. First, assuming that the system operator succeeds in keeping the frequency/voltage constant, any occurrence at any point of the network that causes a variation in supply relative to demand, whether predicted or not, is instantly transferred to spot prices. When an supply-demand imbalance is significant and transitory, there is an abrupt change in the price followed by a quick reversion to the trend. This is an important explanation for the existence of frequent spikes in electricity spot prices. Second, since each power plant generates electricity from a very specific fuel (e.g. water, gas, oil, uranium, wind, etc) their marginal costs are very sensitive to the availability of these inputs. The non-storability of electricity implies 
that any major variations in input prices shift the supply curve immediately, contributing to the volatility observed in electricity prices. Third, demand itself usually has very strong patterns. Consumption of electricity is clearly affected by the time of the day, by the day of the week and by the month of the year. The same is true of the availability of inputs. Since these patterns in demand and supply are quickly transferred to prices, we commonly observe patterns in electricity prices as well. Most empirical studies incorporate one or more of these three basic features (spikes, volatility and strong seasonality) to model electricity spot prices.

The existence of spikes and high volatility in electricity spot prices represents a challenge for risk management in the industry. The task is even harder due to the incomplete nature of electricity markets. That is, the number of securities available in electricity markets is far less than necessary to optimally insure against future risks and to target a desirable and feasible level of consumption in each state of nature. ${ }^{6}$ This means that complex financial arbitrage and hedge strategies are very difficult to implement at a relevant scale due to the existence of very few large, organized, liquid and assorted electricity derivative markets. ${ }^{7}$

Further, due to non-storability, the 'cash and carry' arbitrage argument (used to price forward/futures contracts for most commodities) does not apply for electricity. In other words, electricity derivatives hardly ever fit regular financial models. In practice, electricity firms often resort to bilateral and over-the-counter (OTC) forward contracts to offset their risks. These forward markets usually trade considerable amounts of electricity and are almost always dominated by players directly involved in the buying or selling of wholesale electricity. As examples of the relevance of OTC forward transactions in electricity markets, according to Meade and

\footnotetext{
${ }^{6}$ See Heaton and Lucas (1996) for a formal discussion about incomplete markets and consequences to risk sharing and asset pricing.

${ }^{7}$ The Norwegian electricity market (Nordpool) is usually referred as one of the most developed and complete. However, it cannot be compared to regular commodity markets such as oil in terms of completeness.
} 
O'Connor (2009), the volume of outstanding OTC forward contracts is greater than $100 \%$ of the instantaneous physical volume traded in both the United Kingdom and Australia. According to the same paper, the PJM market is also predominantly made through OTC markets. The acquisition of companies in both the generation and the retail sectors by the same firm (vertical integration) is a long-term contractual arrangement which can also be viewed as an alternative mechanism by which electricity firms hedge their risk. According to a survey undertaken by the NZ electricity commission in 2008 (UMR (2008)), around only 25\% of the electricity sold by generators is hedged through forward/futures markets. As we analyze later in the thesis, this is a potential consequence of the high degree of vertical integration in the market.

The analysis of strategic interactions between agents that face such an intricate array of incentives is very complex. The relationship between contracts (including both forward contracts and vertical integration arrangements) and market power arise as a particularly important and intriguing topic. The evaluation of anti-competitive behavior, the causes and the consequences of vertical integration and the relationship between contracts and market power have been the subject of an already vast literature in industrial organization. ${ }^{8}$ On the other hand, on the financial front, the idiosyncrasies of electricity spot prices and electricity risk management have also stimulated the publication of several articles. ${ }^{9}$ However, sparse

\footnotetext{
${ }^{8}$ Joskow (2005), Bushnell, Mansur, and Saravia (2008) and Meade and O'Connor (2009) offer a broad review about the status quo of the literature over vertical integration. On the other hand, papers such as Allaz and Villa (1993), Newbery (1998), Green (1999), Ferreira (2003), Mahenc and Salanie (2004), Liski and Montero (2006), Green and Le Coq (2006) and Bushnell (2007) deal with the relationship between market power and contracts. In particular, Allaz and Villa (1993), Newbery (1998), Green (1999) and Bushnell (2007) using Cournot or supply function equilibria (SFE) frameworks observe the importance of forward contracts in reducing market power. On the other hand, Ferreira (2003), Mahenc and Salanie (2004), Liski and Montero (2006) and Green and Le Coq (2006) find opposite results either using Bertrand models or focusing on the the dynamic aspects of contracts.

${ }^{9}$ See Falbo, Felletti, and Stefani (2010), Geman and Roncoroni (2006) and Cartea, Figueroa, and Geman (2009) for literature reviews on both electricity risk management and spot price modeling.
} 
effort has been made to relate both fields.

Demand-side and supply-side risks are crucial determinants of hedging decisions in electricity industries. There is a consensus that long-term arrangements have an impact in oligopolistic spot prices. There is also agreement that electricity spot prices and electricity derivatives are somehow linked. In particular, a large portion of electricity transactions are established in forward and future markets. We propose in this thesis a coherent and formal framework to relate these issues and explain the relationship between forward prices, risk and market power, to analyze vertical integration in terms of risk and real options, and to explore the determinants of hedging decision in a realistic setup.

\subsection{Thesis outline}

The objective of this thesis is to develop the link between financial and electricity models. More precisely, to study how volatility and market structure interact to affect the electricity market outcomes in a realistic model setup.

We develop a model that takes into account uncertainty, uniform-price auctions, oligopoly and vertical integration to analyze the relationship between concentration, risk and forward prices. We use the same framework to understand how the decision to increase vertical integration is affected by the demand-side risk and market power. Lastly, we develop a threeperiod equilibrium framework to understand how risk, market power and vertical integration affect hedging decision under the assumption that electricity markets are incomplete.

The thesis comprises five chapters besides the introduction and conclusion. Chapter 2 discusses relevant concepts and assumptions and develops the basic model of the thesis. An electricity equilibrium model that takes into account short-term uncertainty, market power in the wholesale market, forward contracts and uniform-price auctions is presented. A link 
between the instantaneous matching of supply and demand and the dynamics of supply and demand is established through the inclusion of a state variable vector that includes cost and demand shifters. This vector can represent variables associated with input prices (e.g. oil, gas and uranium), climate (e.g. temperature and water inflows), economic activity (e.g. GDP growth and income) and institutions. The chapter derives a linear equilibrium relationship between spot price, contracts and state variables.

Chapter 3 extends the basic model to incorporate the possibility that some firms operate in both the generation and retail markets (the existence of the so-called gentailers). The chapter derives two spot price models, each with different assumptions regarding the nature of uncertainty. Under simplifying assumptions, this chapter also shows that forward contracts do not affect spot prices in highly vertically integrated markets. This result holds even in the case where there are mismatches in a gentailer's position in the generation and retail markets. We also show that, under the assumption of highly vertically integrated markets (even where some gentailers are predominantly retailers and others are predominantly generators), spot prices can be described by a linear function of state variables.

Chapter 4 develops the hybrid approach, incorporating the structure and characteristics of electricity markets, to analyze how forward prices relate to supply-side and demand-side risks and market concentration. Specifically, we develop a closed-form solution for the prices of forward contracts in highly vertically integrated markets (e.g. NZEM, IEM or GEM). The effects that the number of generators and volatility have on the forward curve are then evaluated. Theoretical results are empirically tested and applied to the NZEM.

Chapter 5 analyzes the investment decision associated with vertical integration. Gentailers' decisions to increase their participation in the retail market involves sunk costs. In an uncertain environment the option to postpone this decision has a value. Taking this into account, we develop a 
model that establishes the relationship between parameters of the electricity market, demand uncertainty, and the option to wait for the investment. The model also determines the level of demand which triggers investment (an optimal investment rule). As mentioned before, vertical integration is a relevant phenomenon in electricity markets. Instead of studying vertical integration from a strategic perspective, we innovate by analyzing its determinants from an investment perspective. In particular, we consider the role of uncertainty, flexibility and market power in this decision.

Chapter 6 develops a three-period model and discusses the role of forward contracts as an endogenous variable. As was the case in previous chapters, the assumptions comprise oligopoly, uniform-price auction and the possibility of forward markets and vertical integration. The model assumes that firms decide their contract position in the first period. In the second period, state variables are revealed and generators decide their optimal supply schedule under short-term uncertainty about the final demand. In the last period, the final demand is revealed and the spot market clears. The chapter analyzes the impact of demand-side and supply-side risks over the forward market equilibrium. The impact of risk on generators/gentailers' spot market power is also studied.

We conclude the thesis by arguing that hybrid models have an important and promising role in the analysis of both derivatives prices and investment decisions. Last but not least, when analyzing the relationship between contracts and market power, most papers either take forward prices/contracts as given or do not consider uncertainty in their models. First, we stress the importance of not ignoring risk, the main determinant of hedging, in the discussion about the role of long-term contracts as instruments to promote competitive spot prices in electricity markets. We show that popular measures of wholesale oligopolistic conduct such as the expected mark-up (difference between spot prices and marginal production costs) can be exogenously affected by volatility. Under simplifying assumptions we show that forward/futures contracts do not affect 
spot prices in highly vertically integrated markets. We take into account both demand-side and supply-side risk and show that, even in this limit case, concentration in the wholesale market can positively shift and rotate the forward curve. If changes in the number of generators/gentailers can affect forward prices, one must expect that they can affect hedging decision. In other words, the relationship between market power and forward contracts must be considered in both directions. 


\section{Chapter 2}

\section{Basic model}

\subsection{Model set-up and definitions}

This chapter presents and discusses the electricity market model developed by Hortacsu and Puller(2005, 2008). Their model is modified to include a general vector of state variables affecting demand and supply. The equilibrium spot price formulation here analyzed guides the remaining chapters of the thesis.

The model assumes the following structure. The electricity market has $N$ players made of $K$ generators and $R$ retailers. The wholesale market is oligopolistic, in the sense that there are only a few electricity generators, who must carefully consider the actions of their rivals in setting prices. The wholesale spot price at a given time is determined through a uniform price auction, where generators submit an individual supply schedule and an auctioneer clears the market. The sources of uncertainty at the time of the auction for a given generator are the rival's electricity contract positions and demand oscillation. The bidder's utility function is generic ${ }^{1}$. Both the contract positions and forward prices are considered to be exogenous. The aggregate consumer's demand and generators' cost functions

\footnotetext{
${ }^{1}$ In fact, as defined later, the utility function is quasi-concave and twice differentiable.
} 
are influenced by a given set of state variables assumed as known at the moment of the auction. These state variables drive the stochastic behavior of demand and supply through time. All these assumptions will now be formalized and motivated.

Definition 2.1.1. State variables are represented by the L-dimension state variables vector $\vec{W}_{t}=\left\{w_{1 t}, w_{2 t}, \ldots, w_{L t}\right\}$ which is assumed to be exogenous and known by all firms at time $t$.

Here we define consumer demand and generator cost shifters. These shifters are assumed as known at time $t$. For example, in chapter 4 , they are responsible for the stochastic behavior of price through time. in other words, players are assumed to make their decision and the market equilibrium is set given all the information available at $t$.

Definition 2.1.2. The consumers' aggregate demand at time $t$ is defined by:

$$
\tilde{D}_{t}\left(p_{t}^{R}, \vec{W}_{t}, \varepsilon_{t}\right)=D_{t}\left(p_{t}^{R}, \vec{W}_{t}\right)+\varepsilon_{t}
$$

Retail price $p_{t}^{R}$ is assumed to be exogenous and $\varepsilon_{t}$ represents short-term demand's fluctuations.

Aggregate demand has two components. The first is represented by the function $D_{t}$ which is affected by the price charged to final consumers (retail price) and by demand drivers. The second is $\varepsilon_{t}$ which corresponds to parallel shocks to the demand curve. The probability distribution of $\varepsilon_{t}$ is general. Notice that, by definition, $\vec{W}_{t}$ and $p_{t}^{R}$ are known at time $t$ by all players but $\epsilon_{t}$ is not. At the moment of the auction, players use all information available to infer demand and costs. However, there is still a residual uncertainty about the demand at the time of the power delivery given by $\epsilon_{t}$. The timing of the model is explained in more details later.

Frequently, the literature considers the state variable as the observed demand itself $D_{t}=w_{1 t}$. However, we can also think in terms of demand 
shifters such as income, economic activity, institutional changes, seasonality or climate factors. The assumption of exogeneity of $p_{t}^{R}$ is a good approximation for electricity markets for two reasons. First, retail prices are frequently regulated. Second, even when retail prices are freely determined, contracts between retailers and customers usually have a longterm nature. In other words, it is not reasonable to assume that retailers react to each instantaneous oscillation in the spot market when deciding the price they charge consumers. ${ }^{2}$

Definition 2.1.3. $Q C_{i t}$ is firm i's contracted quantity of electricity to deliver (buy if negative) at time $t$, for all $i=1,2, \ldots, N . P C_{i t}$ refers to the price paid for these contracts.

Definition 2.1.4. The other firms' correspondences $\left(Q C_{j t}, P C_{j t}\right) \forall j \neq i$ are unknown by firm $i$.

The quantity $Q C_{i t}$ is defined as the total amount of electricity that firm $i$ is forward contracted to deliver (or to buy if negative) at time $t$. In reality, we could think as $Q C_{i t}$ representing firm $i$ 's portfolio of forward contracts maturing at $t$, negotiated at different times in the past. In this case, price $P C_{i t}$ would be a weighted average forward price of this portfolio.

Forward contracts constitute an important part of competitive electricity wholesale markets. Wholesalers, retailers and gentailers frequently manage their spot price risk trading significant amounts of forward contracts of different maturities in over the counter (OTC) markets. Because of the bilateral nature of OTC markets, market information is rarely available even for participants. Firms are often unaware of rivals' contract positions at a particular point in time. On the other hand, electricity markets are often characterized by a lack of relevant and liquid future exchanges. Therefore, existence of non marked-to-market forward prices and absence of information about rivals' average forward prices are very common features

\footnotetext{
${ }^{2}$ In reality, wholesale and retail demand can can present different features. In fact retail and industrial prices are frequently not the same. However, to keep the model simple, we consider just one aggregate demand affected by retail prices.
} 
in electricity markets. Frequently, firms' forward positions in a particular time correspond to a complex portfolio, characterized by overlapping contracts established at different periods and prices.

Definition 2.1.5. Function $S_{i t}\left(p, Q C_{i t}, \vec{W}_{t}\right)$ represents generator $i$ 's supply for all $i=1, \ldots, K$. Define $S_{t}=\sum_{i=1}^{K} S_{i t}$ as the aggregate supply.

Supply is affected not only by the spot price $p$ and cost shifters. Generators and also gentailers decide on their supply schedule taking into account the quantity of electricity they are committed to supply at time $t$ by previously arranged contracts.

Definition 2.1.6. The total cost of each generator $i$ in time $t$, for all $i=1,2 \ldots, K$, is $C_{i t}$ which is a function $C_{i t}\left(S_{i t}, \vec{W}_{t}\right)$ of the firm supply $S_{i t}$ and the vector $\vec{W}_{t}$. The marginal cost $M C_{i t}\left(S_{i t}, \vec{W}_{t}\right)$ is the partial derivative of $C_{i t}$ with respect to $S_{i t}$. Also, $C_{i t}$ is twice continuously differentiable and $\frac{\partial M C_{i t}}{\partial S_{i t}} \geq 0$.

We assume that generators have a well behaved cost function shifted by exogenous state variables. This assumption addresses the potential impact of cost shifters (e.g. capacity availability, temperature, precipitation and prices, or shadow prices, of inputs such as gas, fuel or water) in the marginal costs of the generators. Abrupt increases in the marginal cost of generation associated with non-storability of electricity is well documented as a reason for the existence of the so-called spikes (see e.g. Deng (2000) and Hughes and Parece (2002)) . The above cost formulation is flexible enough to capture this phenomenon.

Definition 2.1.7. The market clearing wholesale price $p_{t}^{c}$ must equate aggregate demand and aggregate supply. $\sum_{i=1}^{K} S_{i t}\left(p_{t}^{c}, Q C_{i t}, \vec{W}_{t}\right)=\tilde{D}_{t}\left(p_{t}^{R}, \vec{W}_{t}\right)$

Firms simultaneously submit continuous supply schedules $\hat{S}_{i t}{ }^{3}$. Con-

\footnotetext{
${ }^{3}$ The fact that supply bids are assumed as such continuous functions simplifies the results. This assumption is adopted as a theoretical benchmark in Hortacsu and Puller (2008) and in the large Supply Function Equilibrium literature originated from Klemperer and Meyer (1989). In reality, however, bids are discrete and Von der Fehr and Harbord (1993), and Kastl(2006, 2008) question the smoothness assumption and study the consequences of constrained bidding.
} 
sidering each firm's bid, the auctioneer computes the equilibrium price $p_{t}^{c}$ that satisfies the market clearing condition. Therefore, at the moment of the auction, from the perspective of firm $i$, the uncertainty in price is due to the uncertainty about the short-term demand oscillation $\varepsilon$ and the contract positions of rival firms and their respective prices $\left\{Q C_{j t}, P C_{j t}, j=\right.$ $1 \ldots N, j \neq i\}$.

Definition 2.1.8. Firm i's ex-post profit upon the realization of market clearing price is:

$$
\pi_{i t}=S_{i t}\left(p_{t}^{c}, Q C_{i t}, \vec{W}_{t}\right) p_{t}^{c}-C_{i t}\left(S_{i t}\left(p_{t}^{c}, Q C_{i t}, \vec{W}_{t}\right), \vec{W}_{t}\right)-\left(p_{t}^{c}-P C_{t}\right) Q C_{i t}
$$

The payoff of generators consists of an operating result $\left(p_{t}^{c} S_{i t}-C_{i t}\right)$ and a financial revenue/loss $\left(P C_{i t}-p_{t}^{c}\right) Q C_{i t}$ from forward market transactions.

Definition 2.1.9. The conditional cumulative distribution function of market clearing price $\left(p^{c}\right)$ realizations is:

$$
H_{i t}\left(p, \hat{S}_{i t}(p) ; Q C_{i t}, \vec{W}_{t}\right) \equiv \operatorname{Pr}\left(p_{t}^{c} \leq p \mid Q C_{i t}, \vec{W}_{t}, \hat{S}_{i t}(p)\right)
$$

where $\hat{S}_{i t}(p)$ is the supply schedule submitted by generator $i$ at time $t$.

As characterized by Wilson (1979) and explored by Hortacsu and Puller (2008), we can establish a Bayesian-Nash equilibrium by defining a probability measure over the realizations of the market clearing price from the perspective of generator $i$, conditional on generator $i$ 's private information about his contracts $\left(Q C_{i t}, P C_{i t}\right)$ and the fact that generator $i$ submits the supply schedule $\hat{S}_{i t}$ while its generation competitors are playing their equilibrium bidding strategies $\left\{S_{j t}\left(p, Q C_{j t}, \vec{W}_{t}\right), j=1 \ldots K, j \neq i\right\}$.

Definition 2.1.10. $U_{i}(\pi)$ is the utility enjoyed by the bidder (generator) $i$ from making $\pi$ dollars of profit. $U_{i}(\pi)$ is twice differentiable utility function and $U_{i}^{\prime}(\pi)>0$ and $U_{i}^{\prime}(\pi) \leq 0$. 
A general utility function formulation permits admission of risk aversion of the bidder when participating in the auction. In particular, utilities matter in a context of incomplete markets such as electricity. Preferences are discussed more fully in chapter 6 .

Here we will also assume that generator $i$ 's bidder when deciding the bid schedule $\hat{S}_{i t}(p)$ has utility maximizing behavior.

\subsection{Equilibrium results}

Taking all the previous definitions into account, the bidder $i$ 's expected utility maximization problem is:

$$
\begin{aligned}
\max _{\hat{S}_{i t}(p)} \int_{\underline{p}}^{\bar{p}} \quad & U\left[\hat{S}_{i t}(p) p-C_{i t}\left(\hat{S}_{i t}(p), \vec{W}_{t}\right)\right. \\
& \left.-\left(p-P C_{t}\right) Q C_{i t}\right] d H_{i t}\left(p, \hat{S}_{i t}(p) ; Q C_{i t}, \vec{W}_{t}\right)
\end{aligned}
$$

The integral is taken over all possible realizations of the market clearing price $\left(\varepsilon_{t}, Q C_{j t} ; Q C_{i t}, \vec{W}_{t}\right)$, for all $j \neq i$, weighted by the probability density $d H\left(p, S_{i t}^{*}(p) ; Q C_{i t}, \vec{W}_{t}\right)$. In other words, by offering to supply at a lower price, the bidder increases the likelihood that he will supply a larger quantity; whereas, by offering to supply at a higher price, the bidder increases the likelihood that he will supply a smaller quantity but at a higher price. Taking into account the inherent probability distribution of the clearing price and his own risk aversion, a rational bidder optimizes this tradeoff to maximize his expected utility.

Lemma 2.2.1. Assuming that supply schedules are continuously differentiable and that $S_{i t}^{*}(p)$ is the optimal supply curve of firm $i$ at time $t$, the first order condition (FOC) of the bidder's (generator) maximization problem is:

$$
p-M C_{i t}\left(S_{i t}^{*}(p), \vec{W}_{t}\right)=\left[S_{i t}^{*}(p)-Q C_{i t}\right] \frac{H_{S}\left(p, S_{i t}^{*}(p) ; Q C_{i t}, \vec{W}_{t}\right)}{H_{p}\left(p, S_{i t}^{*}(p) ; Q C_{i t}, \vec{W}_{t}\right)} .
$$


Where

$$
\begin{aligned}
& H_{p}\left(p, S_{i t}^{*}(p) ; Q C_{i t}, \vec{W}_{t}\right)=\frac{\partial}{\partial p} \operatorname{Pr}\left(p_{t}^{c} \leq p \mid Q C_{i t}, \vec{W}_{t}, S_{i t}^{*}(p)\right) \\
& H_{S}\left(p, S_{i t}^{*}(p) ; Q C_{i t}, \vec{W}_{t}\right)=\frac{\partial}{\partial S} \operatorname{Pr}\left(p_{t}^{c} \leq p \mid Q C_{i t}, \vec{W}_{t}, S_{i t}^{*}(p)\right)
\end{aligned}
$$

Proof. appendix A.

Notice that the risk aversion of the bidder does not influence equation (2.4). This result follows from the deterministic nature of all non-control variables of the bidder's maximization problem at time $t$. The bidder's problem solution in every state of nature is attainable and produces a supply schedule that is a monotonically increasing function of price. In other words, the bidder chooses an optimal supply for each state of nature given by $\varepsilon_{t}$.

As pointed out by Hortacsu and Puller (2008), $H_{p}$ is the 'density' of the market clearing price when firm $i$ bids $S_{i t}^{*}(p)$. The derivative $H_{S}$ captures the market power of $i$ and can be interpreted as the 'shift' in the probability distribution of the market clearing price, due to a change in $S_{i t}^{*}(p)$. This derivative is always nonnegative, because an increase in supply weakly lowers the market clearing price, which weakly increases the probability that the market clearing price is lower than a given price $p$.

This formula is consistent with market power or, in other words, the existence of declining residual demand curves. Each bidder is independently selecting his bid to maximize profits based on his estimate of the residual demand curve he faces. Equation 2.4 also implies that the existence of forward contracts mitigates the market power of electricity producers. Observe, however, that for a competitive market we have $p=M C$ independently of the quantity contracted by the generators (as $H_{S}=0$ ).

Equation (2.4) raises three complications. First, as observed by Hortacsu and Puller (2008), its empirical implementation requires the estimation of $H_{i t}$ for each bidder $i$, in every period $t$ which is a complex econometric problem. Second, the computation of equilibrium strategies is a 
complicated task because $H_{i t}$ is determined endogenously through the market clearing condition and depends on the joint distribution of contract positions and the distribution of demand noise. ${ }^{4}$

To understand the possibility of multiple equilibria under the inelastic demand assumption, observe that proposition 2.2.1 becomes a typical supply function equilibrium model (SFE) when forward contracts are not considered. ${ }^{5}$ Anderson and Philpott (2002) show that when demand is inelastic and forward markets are not considered $\left(Q C_{i}=0, \forall i=1,2, \ldots, N\right)$, generators with identical and convex cost functions have an infinite number of choices that yield symmetric equilibria. Different equilibria could also imply significantly different returns for generators. ${ }^{6}$ In a general context, as observed by Holmberg and Newbery (2010), the unique equilibrium would be determined by price caps and capacity constraints under perfectly inelastic demand.

As shown by Hortacsu and Puller (2008), the characterization of equilibrium strategies is greatly simplified when the functional form of the firm $i$ supply strategy is additively separable in price $p$ and quantity contracted $Q C_{i}$, in which case changes in exogenous variables such as $Q C$ or $\vec{W}$ shift the equilibrium supply strategies but do not rotate them. Notice,

\footnotetext{
${ }^{4}$ See Hortacsu and Puller (2008) pages 93 and 94. Third, without further assumptions, equation (2.4) is prone to multiple equilibria. Anderson and Philpott (2002) point out that this is a particularly relevant problem when demand is assumed to be inelastic to wholesale prices (definition 2.1.2).

${ }^{5}$ The SFE approach was originally developed by Klemperer and Meyer (1989) and first applied to the electricity market by Green and Newbery (1992) and Bolle (1992). Holmberg and Newbery (2010) offer a broad review on the SFE literature. These authors define the SFE model as a game-theoretic model of competition in wholesale markets that assume that each generator chooses its offer curve in order to maximize its profit, given demand and offer curves chosen by competitors. The setting of SFE assumes that production costs are common knowledge and that demand is uncertain. It is also assumed that the shock $\varepsilon$ is additive to the demand and that production uncertainties are neglected for strategic producers. Following Hortacsu and Puller (2008), our framework uses exactly the same assumptions plus the assumption of uncertainty about rivals' quantities contracted. With no forward markets, there is no such additional uncertainty and our setting collapses into a typical SFE model.

${ }^{6}$ See Anderson and Philpott (2002) theorem 14, page 486-487.
} 
however, that exogenous variables can change suppliers' price elasticities despite not affecting the suppliers' price derivatives. We show that the same assertion is valid in our framework with state variables $\vec{W}$.

An important caveat, as noticed by Hortacsu and Puller (2008), is that the additive separability is an a priori restriction on bidding strategy. It is not necessarily true that every specification of marginal cost functions and joint distribution of contract quantities will lead to equilibrium strategies of this form. However, the authors test the additive separability assumption for the Ercot market and find that the restriction holds on average across bidders.

Lemma 2.2.2. At any time, suppose supply function strategies $S_{i}\left(p, Q C_{i}, \vec{W}\right)$ are restricted to the additively separable class of strategies:

$$
S_{i}\left(p, Q C_{i}, \vec{W}\right)=\alpha_{i}(p)+\beta_{i}\left(Q C_{i}\right)+\sum_{l=1}^{L} \delta_{l i}\left(w_{l i}\right)
$$

then for a range of prices $p \in[\underline{p}, \bar{p}]$ the first order condition at time t turns to:

$$
p_{t}-M C_{i t}\left(S_{i t}, \vec{W}_{t}\right)=\frac{S_{i t}-Q C_{i t}}{\frac{\partial \sum_{j \neq i} S_{j t}}{\partial p}}
$$

alternatively,

$$
\frac{p_{t}-M C_{i t}}{p_{t}}=-\frac{1}{\epsilon_{i t}\left(q_{i t}\right)}
$$

where $\epsilon_{i t}\left(q_{i t}\right)$ is the elasticity of the net residual demand $q_{i t}$, here defined as $q_{i t}=$ $D_{t}-\sum_{j \neq i} S_{j t}-Q C_{i t}$.

Proof: appendix A.

As posed by Holmberg and Newbery (2010), mark-ups in the real-time market only influence the revenue from sales net of forward contracting. Hence, it is the residual demand net of forward contracts that are relevant 
for a profit maximizing producer. A producer offers positive net-supply with positive mark-ups in the realtime market. If a producer has negative net-supply, i.e. he has to buy back electricity in the real-time market, then he will use his market-power to push down the price. Hence mark-ups are negative for negative net-supply. Mark-ups are zero at the contracting point where net-supply is zero. Therefore, the existence of forward contracts mitigates incentives to bid above marginal costs.

Specifically, in equilibrium, firm $i$ 's supply $S_{i}$ is such that his Lerner index $\frac{p_{t}-M C_{i t}}{p_{t}}$ corresponds to the negative of the inverse of the elasticity $-\frac{1}{\epsilon_{i t}\left(q_{i}\right)}$ of his residual demand $D_{t}-\sum_{j \neq i} S_{j t}$ net of his forward position $Q C_{i t}$. In other words, the elasticity of the net residual demand $q_{i}$ fully explains the wholesaler $i$ 's mark-up.

As discussed before, the result of the proposition above comes from a restriction on the set of admitted supply function strategies. Particularly, by immediate inspection of equation (2.5) one can observe that cost functions with non-linear marginal costs or highly non-linear aggregate demand pose problems with satisfying the assumption of additively separable supply functions. However, proposition 2.2.3 shows that the empirically implementable simplification of quadratic cost functions and linear demand is consistent with additively separable supply strategies.

Given the concavity of the profit function, Hortacsu and Puller (2008) points out that the bid function $S_{i}(p)$ given by equation (2.5) provides a pointwise best-response to every possible realization of the residual demand curve. This also means that a class of additively separable equilibrium strategies are ex post optimal when they exist. That is, seeing other bidders supply functions would not change bidder is choice of supply function. This result also resembles $\operatorname{Wolak}(2000,2003)$. In particular, both papers assume uncertainty in demand, and find that, given certain conditions, and regardless of the residual-demand realization, equation (2.6) holds for each hour of the day and each supplier. ${ }^{7}$ The contribution of

${ }^{7}$ As discussed by Holmberg and Newbery (2010), ex-post optimality is not always 
Hortacsu and Puller (2008) is that they obtain similar results by allowing for firm i's uncertainty about both the demand $(\varepsilon)$ and his rivals' contract positions $\left(Q C_{j}\right)$.

Following Hortacsu and Puller (2005), proposition 2.2.3 shows that there is an example in which a potential equilibrium strategy is analytically characterizable and satisfies the additive separability restriction. In this example, i) both demand and firms' costs are affected by state variables, ii) the demand is inelastic and iii) firms possess linear marginal cost curves (these can be asymmetric across firms). Further, firms are assumed to be restricted to linear supply strategies.

As far as tractability of the equilibrium spot price model is essential to address central subjects of the next chapters such as forward pricing, vertical integration and hedging decisions, we will restrain our analysis to this particular case. Despite its limitations, as we discuss in chapters 4-6, our setting constitutes a considerable improvement over the spot market structural assumptions made in the optimal hedging / forward pricing literature, frequently characterized by perfect competition assumptions or no assumptions at all.

Notice that, in this thesis, Hortacsu and Puller (2008) is expanded by considering the existence of a vector of state variables $(\vec{W})$ that affect demand and/or supply. This vector is the link between the family of static electricity models and the multi-period models of chapter 4-6.

Proposition 2.2.3. Assume that $S_{i}\left(p, Q C_{i}, \vec{W}\right)$ are restricted to the linear class of strategies:

$$
S_{i t}\left(p_{t}, Q C_{i t}, \vec{W} t\right)=\psi+\alpha p_{t}+\beta Q C_{i t}+\sum_{l=1}^{L} \delta_{j}\left(w_{j t}\right)
$$

possible. In more general settings, producers can choose the best expected outcome before knowing the shock, but might have chosen a different offer curve once they know the actual shock. For example, Wilson (2008) analyzes SFE in a transmission network with multiple nodes where demand shocks are multi-dimensional. In this case, it is generally not possible to find an ex-post optimal SFE. 
If (i) there are a fixed number $K>2$ generators in the market, (ii) marginal cost functions are linear and symmetrical between firms in the market $\left(M C_{i t}=\right.$ $a+b S_{i t}+\sum_{j=1}^{L} \rho_{j} w_{j t} \forall i=1,2, \ldots N$ and $b>0$ ) and (iii) the aggregate demand is linear with constant retail price $\left(D_{t}\left(p_{t}^{R}, \vec{W}_{t}\right)=c-\kappa_{o} p_{t}^{R}+\sum_{j=1}^{L} \kappa_{j} w_{j t}+\varepsilon_{t}\right)$ then there is a unique symmetric Bayesian-Nash equilibrium, if such an equilibrium exists. Where:

$$
S_{i t}^{*}=-\frac{a(K-2)}{b(K-1)}+\frac{K-2}{b(K-1)} p_{t}+\frac{1}{K-1} Q C_{i t}+\frac{-(K-2)}{b(K-1)} \sum_{j=1}^{L} \rho_{j} w_{j t}
$$

and the clearing wholesale spot price can be rewritten as the following:

$$
p_{t}^{c}=A-B \sum_{i=1}^{K} Q C_{i t}+\sum_{j=1}^{L} C_{j} w_{j t}+(K-1) B \varepsilon_{t}
$$

where

$$
\begin{aligned}
A & =a+b \frac{\left(c-\kappa_{o} p^{R}\right)(K-1)}{K(K-2)} \\
B & =\frac{b}{K(K-2)} \\
C_{j} & =\rho_{j}+b \frac{K-1}{K(K-2)} \kappa_{j}
\end{aligned}
$$

Proof: appendix A.

Proposition 2.2.3 states a simple and linear equilibrium relationship between clearing price and state variables under a realistic electricity market structure. That is, the equilibrium allows for market power in the wholesale market and a uniform-price auction clearing mechanism unlike the usual assumptions of perfect competition and pay-as-bid mechanism. If $K \leq 2$, the results could be not feasible since $A, B$ and $C_{j}$ would be divided by zero for $K=2$. The assumption of $K>2$ is valid for many 
competitive electricity markets (e.g. most of competitive US interconnections, NZEM, Nordpool).

The following results follow from equation (2.7). First, the electricity spot price is affected by the state variables in the expected way. Positive shifts in generators' costs and in aggregate demand increase the spot price and negative shifts have the opposite effect. Second, an increase in the retail price, here assumed as exogenous and deterministic, decreases aggregate demand and consequently decreases the spot price.

The third and most significant aspect of this equation is that players' hedge decisions, here represented by the sum of generator's contracts $\sum_{i=1}^{K} Q C_{i t}$, can have a significant role in the price formation. As discussed before, this means that a large forward position can decrease generators' incentive to exercise market power which, in turn, affects the spot price. 


\section{Chapter 3}

\section{Vertical integration}

\subsection{Motivation}

The model described in chapter 2 assumes that electricity markets are vertically separated. However, electricity firms frequently participate in both the generation (upstream) and retail (downstream) markets simultaneously, which is referred to in the economic literature as vertical integration. This chapter expands the previous model in order to take into account the possibility of vertical integration.

No such thing as a unified theory of vertical integration exists so far. There is an array of theories to explain this rather usual phenomenon in electricity markets. However, as posed by Joskow (2005), virtually all theories of vertical integration arise from the recognition of market imperfections of some type.

Traditional approaches have mostly focused on vertical integration as an arrangement associated either with a strategic move to increase market power (downstream and/or upstream) or a response to market power problems already in existence. ${ }^{1}$ Other alternative approaches focus on fric-

\footnotetext{
${ }^{1}$ In this case, as explained by Joskow (2005), vertical integration could be an optimal response to costs of successive monopolies (Tirole (2000) chapter 4), it could enhance price discrimination (Perry (1978)), or it could be used to soften competition by increasing ri-
} 
tions such as transaction costs and incompleteness of contracts to study vertical integration from an organizational perspective. Unlike most the traditional approaches that consider the act of vertical integration as theoretically costless, alternatives such as the "Property Rights" approach and Transaction Cost Economics see vertical integration as a reaction to costs and benefits of internal organization and to inefficiencies of market transactions. ${ }^{2}$ Chapter 3 considers two different equilibrium formulations for the spot market: the first will be used to build hybrid pricing models (chapters 4-5) and the second will be used for optimal hedging (6). Both models treat vertical integration as a long-term hedging arrangement.

The differences between vertical integration and long-term forward contracts in our formulations is related to the way they are affected by uncertainty. Given a specific retail market share $m_{i}$ and a retail price $P^{R}$, the retail profit of a gentailer $i$ will immediately follow the variations of aggregate demand. On the other hand, the outstanding quantity contracted $Q C_{i t}$ at time $t$ is assumed fixed. In other words, in opposition to forward contracts, vertical integration fully hedges against risks embedded in demand ${ }^{3}$. Observe that this assumption about vertical integration is related to the transaction costs / property rights' literature, in the sense that the differences between the two arrangements occur due to the incompleteness of the market. If we could costlessly hedge all the uncertainty in demand through forward contracts, in each instant $t$, both arrangements would be virtually equal for hedging purposes.

The first equilibrium model developed in this chapter assumes that the only source of uncertainty faced by bidders (generator/gentailer) is the

vals' costs and/or foreclosure potential competitors(Aghion and Bolton (1987), Ordover, Saloner, and Salop (1990) and Hart, Tirole, Carlton, and Williamson (1990)). For a more didactic approach, see Schmalensee, Armstrong, and Porter (2007) chapter 33.

${ }^{2}$ See, for example, Williamson (1971), Klein, Crawford, and Alchian (1978), Klein and Leffler (1981), Williamson (1983), Williamson (1985), Grossman and Hart (1986), Hart and Moore (1990), Hart and Hart (1995), Klein (2000) and Williamson (2000).

${ }^{3}$ The models developed in this chapter assume that, for a given period $t$, both $Q C_{i t}$ and $m_{i t}$ were already defined and, therefore, are exogenous parameters. These assumptions will be partially relaxed in later chapters. 
quantity contracted by their rivals. It implicitly assumes that the oscillations in demand $(\varepsilon)$ between the time of the auction and the time of delivery are negligible. On the other hand, it considers that a given firm does not know for sure his rivals' outstanding contracts. This assumption is particularly appropriate for developing the hybrid pricing models of chapter 4 since they focus on longer-term risks associated with forward and real options pricing. As we see in the next chapter, these longer-term risks are driven by the stochastic process associated to the state variables.

The second formulation assumes that the rivals' quantity contracted is absolutely known at time $t$ but there is a residual uncertainty $(\varepsilon)$ about the demand at the same time. This alternative formulation is used in the threeperiod model of optimal hedging under vertical integration of chapter 5. This fundamental assumption is appropriate in the context of optimal hedge modeling. Since players behave rationally and there is no asymmetric information in the market, each player knows the optimal contract strategy of all his rivals. Therefore, there is no reason to assume the existence of uncertainty about $Q C_{j t}$ in this three-period world. Notice that, differently from the first model, this second framework allows for uncertainty in both state variables and post-auction disturbances in demand. These models are formalized in the next sections.

\subsection{General assumptions}

Assume $N$ total firms made up of $K$ generators, $R$ retailers and $I$ gentailers, where $I=K+R-N$. That is, gentailers are included in both retailers and generators groups. The retailers' revenues are determined by an exogenous retail price and by their market shares. Consider that definitions 2.1.1, 2.1.3, 2.1.5 and 2.1.6 hold. The consumers' aggregate demand at time $t$ is defined by $\tilde{D}_{t}$. Since the participation of generators in the retail market also drives their supply decision, the characterization of each retailer's individual demand becomes relevant. 
Definition 3.2.1. Retailers' demand (gentailer or pure retailer) is defined as $m_{i} \tilde{D}_{t}\left(p_{t}^{R}, \vec{W}_{t}\right), \forall i=1,2, \ldots, R$. Here $m_{i}>0$ is the given market share of retailer $i$ and $\sum_{i=1}^{R} m_{i}=1$, since gentailers are included in retailers. By construction, $\sum_{i=1}^{R} m_{i} \tilde{D}_{t}\left(p_{t}^{R}, \vec{W}_{t}\right)=\tilde{D}_{t}\left(p_{t}^{R}, \vec{W}_{t}\right)$.

A retailer's demand is assumed to be a fixed proportion of the total consumers' demand and, by construction, the total retailers' demand must be equal to the aggregate consumers' demand. The exogeneity of $m_{i}$ reflects the idea that the retail market shares are relatively fixed. It is certainly reasonable to assume that at the moment of an auction the retail market share is known and exogenous. In reality, contract arrangements between retailers and final consumers are relatively stable in comparison to the strong variations observed in both demand and generation inputs. Therefore, this assumption is a good approximation for the short or medium-term.

Definition 3.2.2. The market clearing wholesale price $p_{t}^{c}$ must equate aggregate demand and aggregate supply. $\sum_{i=1}^{K} S_{i t}\left(p_{t}^{c}, Q C_{i t}, \vec{W}_{t}\right)=\sum_{i=1}^{R} m_{i} \tilde{D}_{t}\left(p_{t}^{R}, \vec{W}_{t}\right)=$ $\tilde{D}_{t}\left(p_{t}^{R}, \vec{W}_{t}\right)$

Again, firms simultaneously submit continuous supply schedules $\hat{S}_{i t}$ and the auctioneer computes the equilibrium price $p_{t}^{c}$ that satisfies the market clearing condition.

Definition 3.2.3. Gentailer $i$ 's ex-post profit upon the realization of the market clearing price is (where $m_{i}=0$ for pure generators and $m_{i}>0$ for gentailers):

$$
\begin{aligned}
\pi_{i t}= & S_{i t}\left(p_{t}^{c}, Q C_{i t}, \vec{W}_{t}\right) p_{t}^{c}-C_{i t}\left(S_{i t}\left(p_{t}^{c}, Q C_{i t}, \vec{W}_{t}\right), \vec{W}_{t}\right) \\
& +m_{i}\left(p_{t}^{R}-p_{t}^{c}\right) \tilde{D}_{t}\left(p_{t}^{R}, \vec{W}_{t}\right)+\left(P C_{i t}-p_{t}^{c}\right) Q C_{i t}
\end{aligned}
$$

There are three possible sources of payoff for electricity companies $i=$ $1 \ldots N$ : operating profit from generation activity $\left(S_{i t}-C_{i t}\right)$, operating profit from retail activity $m_{i}\left(p_{t}^{R}-p_{t}^{c}\right) \tilde{D}_{t}$ and financial revenue $\left(P C_{i t}-p_{t}^{c}\right) Q C_{i t}$ from forward market transactions. The differences between financial and 
physical transactions were explained in the introduction. As defined before, gentailers are characterized by participating in both generation and retail markets. Therefore, they have operating profits (or losses if negative) in both activities.

Definition 3.2.4. Pure retailer $i$ 's ex-post profit upon the realization of market clearing price is:

$$
\pi_{i t}=m_{i}\left(p_{t}^{R}-p_{t}^{c}\right) \tilde{D}_{t}\left(p_{t}^{R}, \vec{W}_{t}\right)-\left(p_{t}^{c}-P C_{i t}\right) Q C_{i t}
$$

Notice that, as posed by definition 2.1.3, $Q C_{i t}$ may be negative. For example, if pure retailers solely buy electricity in the forward market, they have a negative contract position by our definition. Definition 3.2.4 assumes retailers as passive players in the instantaneous wholesale spot market. That is, a retailer's purchase is totally determined by his exogenous retail market participation $m_{i} \tilde{D}_{t}\left(P_{t}^{R}, W_{t}\right)$. It also means that there are no strategic alternatives considered by pure retailers and the spot market equilibrium is fully determined by supplier strategies and the exogenous aggregate demand. This is a reasonable approximation for most uniformprice auctions in electricity markets, where only suppliers bid and markets are cleared by an auctioneer responsible for matching supply curves to particular electricity demands.

Definitions 2.1.9 and 2.1.10 still hold. Assume that generator/gentailer $i$ 's bidder when deciding the bid schedule $\hat{S}_{i t}(p)$ has utility maximizing behavior. The bidder $i$ expected utility maximization problem is:

$$
\begin{aligned}
& \max _{\hat{S}_{i t}(p)} \int_{\underline{p}}^{\bar{p}} \quad U\left[\hat{S}_{i t}(p) p-C_{i t}\left(\hat{S}_{i t}(p), \vec{W}_{t}\right)+m_{i}\left(p_{t}^{R}-p\right) \tilde{D}_{t}\left(p^{R}, \vec{W}_{t}\right)\right. \\
& \left.+\left(P C_{i t}-p\right) Q C_{i t}\right] d H_{i t}\left(p, \hat{S}_{i t}(p) ; Q C_{i t}\right),
\end{aligned}
$$

As discussed in chapter 2, the integral is taken over all possible realizations of the market clearing price, weighted by the probability density 
$d H_{i t}$. Now we present the models.

\subsection{Model 1: uncertain rivals' contracts}

Consider that the following definitions hold.

Definition 3.3.1. The consumers' aggregate demand at time $t$ is defined by the function $\tilde{D}_{t}=D_{t}\left(p_{t}^{R}, \vec{W}_{t}\right)$. Retail price $p_{t}^{R}$ is assumed to be exogenous.

Definition 3.3.2. The other firms' correspondences $\left(Q C_{j t}, P C_{j t}\right) \forall j \neq i$ are unknown by firm $i$.

Aggregate demand is only affected by the state variables $\vec{W}_{t}$ and the retail price $p_{t}^{R}$. At the time of the auction, the demand function is deterministic. In terms of the model analyzed in the previous chapter, this definition is equivalent to assuming that $\varepsilon$ is negligible. In fact, uniformprice auctions used to clear electricity spot markets have a very short-term horizon. Bids into uniform price electricity auctions are made for delivering energy close to dispatch. In markets such as the NZEM, the bid can be modified until two hours to the delivery time. The more significant source of uncertainty for a specific bidder at the time of the auction is the hedging position of his rivals.

Lemma 3.3.1. In equilibrium, assuming again that supply schedules are continuously differentiable and that $S_{i t}^{*}(p)$ is the optimal supply curve of firm $i$ at time $t$, the first order condition for the bidder's (gentailer/generator) maximization problem is:

$$
p-M C_{i t}\left(S_{i t}^{*}(p), \vec{W}_{t}\right)=\left[S_{i t}^{*}(p)-Q C_{i t}^{*}-m_{i} D_{i t}\left(p_{t}^{R}, \vec{W}_{t}\right)\right] \frac{H_{S}\left(p, S_{i t}^{*}(p) ; Q C_{i t}^{*}\right)}{H_{p}\left(p, S_{i t}^{*}(p) ; Q C_{i t}^{*}\right)}
$$

where

$$
H_{p}\left(p, S_{i t}^{*}(p) ; Q C_{i t}^{*}\right)=\frac{\partial}{\partial p} \operatorname{Pr}\left(p_{t}^{c} \leq p \mid Q C_{i t}^{*}, S_{i t}^{*}(p)\right)
$$




$$
H_{S}\left(p, S_{i t}^{*}(p) ; Q C_{i t}^{*}\right)=\frac{\partial}{\partial S} \operatorname{Pr}\left(p_{t}^{c} \leq p \mid Q C_{i t}^{*}, S_{i t}^{*}(p)\right)
$$

Proof: appendix B.

As explained in chapter 2, this result follows from the deterministic nature of all non-control variables and from the fact that the bidder is able to choose an optimal supply for each possible state of nature.

Assume that generator/gentailer $i$ 's net supply is the difference between his supply and his retail position $\left(S_{i t}^{*}(p)-m_{i} D_{i t}\right)$. Equation (3.4) shows that a gentailer's bid behavior is driven ceteris paribus not only by contracts but also by his net supply. In the absence of forward contracts, the bigger the net supply, the bigger the incentive to exert market power in the wholesale market. The generator's participation in the retail market reduces his incentives to bid above marginal cost. If the gentailer is a net retailer $S_{i t}^{*}(p)-m_{i} D_{i t}<0$ he may even bid below his marginal cost.

Lemma 3.3.2. Consider that assumptions of lemma 2.2.2 hold. For a range of prices $p \in[\underline{p}, \bar{p}]$ the first order condition at time $t$ becomes:

$$
p_{t}-M C_{i t}=\frac{S_{i t}-Q C_{i t}^{*}-m_{i} D_{t}}{\frac{\partial \sum_{j \neq i} S_{j t}}{\partial p_{t}}}
$$

Alternatively,

$$
\frac{p_{t}-M C_{i t}}{p_{t}}=\frac{1}{\varepsilon_{i t}^{\prime}\left(q_{i t}^{\prime}\right)}
$$

Where $\varepsilon_{i t}^{\prime}\left(q_{i t}^{\prime}\right)$ is the elasticity of the net residual demand $q_{i t}^{\prime}$, here defined as $q_{i t}^{\prime}=D_{t}-\sum_{j \neq i} S_{j t}-Q C_{i t}^{*}-m_{i} D_{t}$.

Proof: appendix B.

Under the hypothesis of quadratic cost functions and linear demand, the gentailer's supply curve obeys the usual pointwise Lerner index as shown by equation (3.6). 
Specifically, gentailer i's supply $S_{i}$ is such that his Lerner index $\frac{p_{t}-M C_{i t}}{p_{t}}$ corresponds to the inverse of the elasticity $\frac{1}{\varepsilon_{i t}\left(q_{i}\right)}$ of his residual demand $D_{t}-\sum_{j \neq i} S_{j t}$ net of his equilibrium forward position $Q C_{i t}^{*}$ and his participation in the retail market $m_{i} D_{t}$. In other words, the elasticity of the net demand $q_{i}$ fully explains wholesaler $i$ 's market power. This result comes though from the additional assumption of instantaneous perfect inelasticity of aggregate demand $D_{t}$ to wholesale spot prices $p_{t}$ at time $t$.

Proposition 3.3.3. If (i) there are a fixed number $K>2$ generators/gentailers in the market, (ii) marginal cost functions are linear and symmetrical between firms in the market $\left(M C_{i t}\left(S_{i t}, \vec{W}_{t}\right)=a+b S_{i t}+\sum_{j=1}^{L} \rho_{j} w_{j t} \forall i=1,2, \ldots N\right)$ and (iii) the aggregate demand is linear with constant retail price $\left(D_{t}\left(p_{t}^{R}, \vec{W}_{t}\right)=\right.$ $\left.c-\kappa_{o} p^{R}+\sum_{j=1}^{L} \kappa_{j} w_{j t}\right)$ then there is a simple symmetric equilibrium, if such an equilibrium exists, where the clearing wholesale spot price can be rewritten as the following:

$$
p_{t}^{c}=A-B \sum_{i=1}^{K} Q C_{i t}^{*}+\sum_{j=1}^{L} C_{j} w_{j t}
$$

Where

$$
\begin{aligned}
A & =a+b \frac{\left(c-\kappa_{o} p^{R}\right)\left(K-\left(1+\sum_{i=1}^{K} m_{i}\right)\right)}{K(K-2)} \\
B & =\frac{b}{K(K-2)} \\
C_{j} & =\rho_{j}+b \frac{\left(K-\left(1+\sum_{i=1}^{K} m_{i}\right)\right)}{K(K-2)} \kappa_{j}
\end{aligned}
$$

Proof: appendix B.

All the aspects discussed for equation (2.7) remain valid for equation (3.7). Positive shifts in generators' costs and in aggregate demand increase the spot price. An increase in the retail price decreases spot price. The 
sum of generators' contracts $\sum_{i=1}^{K} Q C_{i t}$, play an important role in price formation. ${ }^{4}$

Equation 3.7 also shows that, holding forward contracts constant, an increase in the degree of vertical integration $\left(\sum_{i=1}^{K} m_{i}\right)$ in the market implies a decrease in spot prices. The reason is that more vertically integrated firms have a smaller net supply $S_{i t}-m_{i} D_{i t}$ and therefore less incentives to exert market power in the wholesale market taking contracts as fixed. ${ }^{5}$

Corollary 3.3.4. If $K \rightarrow \infty$ then $p \rightarrow M C$.

There are two exceptions where the hedging decision does not matter for spot price modeling purposes, notwithstanding the size of the electricity hedging market. The first, as posed by the corollary above, refers to the perfect competition case. If the number of generators in the market goes to infinity, the mark-up component of the spot price formation tends to zero. In the limit, we have the competitive result of spot price being equal to generators' marginal cost. In other words, if generators in an electricity market were atomized, wholesale prices would be primarily driven by their marginal costs. In practice, perfect competition does not exist in electricity wholesale markets.

\footnotetext{
${ }^{4}$ The aggregate position of generators $\left(\sum_{i=1}^{K} Q C_{i t}\right)$ is close to zero and does not affect spot prices in two basic situations: (i) electricity markets with a poorly developed forward market and (ii) fully vertically integrated markets as defined later in this chapter. In particular, markets made exclusively of gentailers with the same market share in both the retail and generation markets have little reason to develop forward markets in a large scale, since their wholesale transactions are internally hedged.

${ }^{5}$ Hogan (2010) finds a similar result in a different and deterministic framework, addressing the incentives of gentailers and pure retailers. He finds that the vertically integrated firm has an incentive to compete more aggressively in the retail market than pure retailers. Gans, Wolak, and Carlton (2008) find opposite results considering the role of passive vertical integration. They find that an increase in vertical integration would decrease quantity contracted that would in turn increase spot prices. This result relies strongly that the wholesale and retail businesses are completely separated (independent). This means that the gentailers do not necessarily make a first best decision. Specifically, the forward contract aspects of vertical integration are not considered in the gentailers' supply decision.
} 
Corollary 3.3.5. If $K=N$ then $\sum_{i=1}^{K} m_{i}=\sum_{i=1}^{N} m_{i}=1$ and we have:

$$
p_{t}^{c}=a+\frac{b c-\kappa_{o} p^{R}}{K}+\sum_{j=1}^{L}\left(\frac{b}{K} \kappa_{j}+\rho_{j}\right) w_{j t}
$$

The second concerns the case where forward contracts are fully cleared by generators $\left(\sum_{i=1}^{K} Q C_{i t}=0\right)$. From equation 3.11, this fact applies to markets where $K=N$. That is, where all the firms in the market are generators or gentailers (i.e. all retailers are also generators). In such a case, contracts do not affect the aggregate supply and, consequently, the clearing spot price.

Since this model approximates demand and marginal costs by linear functions, by equation 3.5 the optimal individual supplies are also linear. In particular, they are positively affected by the quantity contracted $\left(Q C_{i t}\right)$. Gentailers can be net wholesalers, net retailers or have the same share in both markets. Intuitively, in order to hedge risks, they are expected to have $Q C_{i t}>0, Q C_{i t}<0$ and $Q C_{i t}=0$ respectively. Therefore, if all the players are gentailers and the aggregate supply is linearly affected by the sum of the generators' outstanding contracts, it is reasonable to expect that the oversupply of net wholesalers will offset the undersupply of net retailers and the aggregate outstanding contracts will have no effect on the aggregate demand.

Define markets where $K=N$ as fully vertically integrated markets. Notice that this definition is broader than the usual definition of full vertical integration in the literature, as it admits mismatch between the participation of an individual gentailer in the generation and retail markets. ${ }^{6}$ Our definition comprises (but it is not limited to) either (i) markets where all the generators are gentailers $(K=I=N)$ or, more strictly, (ii) markets where each generator sells all his production directly to consumers through his retail business (individual full vertical integration).

\footnotetext{
${ }^{6}$ As for example Dixit (1983).
} 
The gentailer dominated electricity markets of Spain, New Zealand or Germany, for example, fit closely to this definition. In New Zealand, the market is dominated by gentailers but some firms present mismatch between their wholesale and retail market shares. In other words, there are big net wholesalers and big net retailers.

Notice that the clearing price is equal to the average marginal cost in fully vertically integrated electricity markets since, in equilibrium, the average supply $\bar{S}$ is equal to the aggregate demand divided by the number of gentailers $\left(\bar{S}=\frac{D}{K}\right)$. This means that individual firms may have market power when $K=N$ but the average mark-up in the market is equal to zero. Equation (3.11) is used in the empirical exercise of chapter 4.

\subsection{Model 2: uncertain short-term demand}

Consider that the following definitions hold.

Definition 3.4.1. The consumers' aggregate demand at time t is defined by:

$$
\tilde{D}_{t}\left(p_{t}^{R}, \vec{W}_{t}, \varepsilon_{t}\right)=D_{t}\left(p_{t}^{R}, \vec{W}_{t}\right)+\varepsilon_{t}
$$

Retail price $p_{t}^{R}$ is assumed to be exogenous and $\varepsilon_{t}$ represents short-term demand fluctuations.

Definition 3.4.2. The other firms' correspondence $\left(Q C_{j t}, P C_{j t}\right) \forall j \neq i$ is known by firm $i$.

Definition 3.4.1 is the same as definition 2.1.2. Demand has two components. The first is represented by the function $D_{t}$ which is affected by the price charged to final consumers (retail price) and by demand shifters. The second is $\varepsilon_{t}$ which corresponds to additive short-term shocks to the demand curve. The probability distribution of $\varepsilon_{t}$ is generic. Unlike the basic model, definition 3.4.2 states that bidders know their rivals' contract position. This assumption will be used in a subsequent chapter to derive a 
three-period model where players strategically decide their optimal hedging and know their rivals' optimal position.

Lemma 3.4.1. Define $S_{t}$ as the aggregate supply. In equilibrium, assume again that supply schedules are continuously differentiable and that $S_{i t}^{*}(p)$ is the optimal supply curve of firm $i$ at time $t$. Consider that the assumptions of lemma 2.2.2 hold. For a range of prices $p \in[\underline{p}, \bar{p}]$ the first order condition at time $t$ becomes:

$$
p_{t}-M C_{i t}=\frac{S_{i t}-Q C_{i t}-m_{i} S_{t}}{\sum_{j \neq i} \frac{\partial S_{j t}}{\partial p_{t}}}
$$

Proof: appendix B.

Recall that $S(p)=\sum_{i=1}^{K} S_{i}(p)$ is the aggregate supply curve. Since the rivals' quantity contracted are known in this model, there is no uncertainty about rivals' supply schedules for a given aggregate demand. Since the demand shock is the only source of uncertainty, both the clearing price $p^{c}$ and the supply schedules $\left(S_{i}\left(p^{c}\right)\right.$ for all $\left.i=1,2, \ldots, K\right)$ are entirely explained by each realization of $\varepsilon$.

Notice that equation (3.10) is different from equation (3.5). In equation (3.5) every player knows the demand at the moment of the auction and the vertical integration arrangement $\left(m_{i} D_{t}\right)$ is equivalent to forward contracts $\left(Q C_{i t}\right)$. Both arrangements are given at $t$ and affect the clearing spot price $\left(p_{t}^{c}\right)$ in a deterministic way. In equation (3.10), the vertical integration arrangement depends on the aggregate supply $\left(m_{i} S_{t}\right)$, which in turn depends on an uncertain demand. In other words, both the rivals' contract position and the rivals' participation in the retail market matter for the optimal supply schedule decision.

Proposition 3.4.2. If (i) there are a fixed number $K>2$ of generators/gentailers in the market, (ii) marginal cost functions are linear and symmetrical between firms in the market $\left(M C_{i t}\left(S_{i t}, \vec{W}_{t}\right)=a+b S_{i t}+\sum_{j=1}^{L} \rho_{j} w_{j t} \forall i=1,2, \ldots N\right)$ and (iii) the aggregate demand is linear with constant retail price $\left(\tilde{D}_{t}\left(p_{t}^{R}, \vec{W}_{t}\right)=c-\right.$ 
$\kappa_{o} p^{R}+\sum_{j=1}^{L} \kappa_{j} w_{j t}+\varepsilon_{t}$ ) then there is a simple equilibrium, if such an equilibrium exists, where the clearing wholesale spot price can be rewritten as the following:

$$
p_{t}^{c}=\frac{1}{\sum_{i=1}^{K} \alpha_{i}}\left(\tilde{D}_{t}-\sum_{i=1}^{K} \psi_{i}+\sum_{i=1}^{K} \beta_{i} Q C_{i t}+\sum_{i=1}^{K} \sum_{j=1}^{L} \delta_{i j} w_{j}\right)
$$

where

$$
\begin{aligned}
\psi_{i} & =\frac{m_{i} \sum_{j \neq i} \psi_{j}+m_{i} \sum_{j \neq i} \beta_{j} Q C_{j}-\sum_{j \neq i} \alpha_{j} a}{1-m_{i}+\sum_{j \neq i} \alpha_{j} b} \\
\alpha_{i} & =\frac{\left(1+m_{i}\right) \sum_{j \neq i} \alpha_{j}}{1-m_{i}+\sum_{j \neq i} \alpha_{j} b} \\
\beta_{i} & =\frac{1}{1-m_{i}+\sum_{j \neq i} \alpha_{j} b} \\
\delta_{i} & =\frac{m_{i} \sum_{j \neq i} \sum_{l=1}^{L} \delta_{j l}-\sum_{j \neq i} \sum_{l=1}^{L} \alpha_{j} \rho_{l}}{1-m_{i}+\sum_{j \neq i} \alpha_{j} b}
\end{aligned}
$$

Proof: appendix B.

Under simplifying assumptions, equation (3.11) shows the relationship between the electricity spot price and the market parameters. Parameters $\psi, \alpha, \beta$ and $\delta$ can be solved numerically. This equilibrium result constitutes the base of the optimal hedging discussed in chapter 5 .

The inclusion of vertical integration, under the assumption of uncertain short-term demand, augments the complexity of suppliers' decisions and reduces the tractability of the model. Without vertical integration all the uncertainty in the generators' profit is reflected in the price distribution. These results, which mirror the classical findings of (Wolak 2000), are built under the possibility of constructing continuous and well behaved supply schedules that take into account an unique and optimal strategic response for each realization of the clearing price.

With vertical integration, the gentailers' payoffs are affected by price and demand uncertainty. Despite the linear relationship between them, 
since all the uncertainty is given by demand (in this case there is no uncertainty about rivals'contracts), this complication poses the need to directly take into account the rivals' optimal supply decision when choosing their own optimal supply. The rivals' supplies are functions of not only their quantity contracted but also of their position in the retail market.

This model is particularly useful for dealing with optimal hedging in the presence of vertical integration. First, it incorporates both the effects of state variables and the effect of short-term demand fluctuations in the hedging decision. Second, it permits different participation in the retail market. Lastly, it allows for the endogenization of quantity contracted and/or vertical integration in a multiperiod framework. Under assumptions of symmetric information and rational agents, it is reasonable to assume that at a specific time $t$, players know their rivals' best hedging responses. 


\section{Chapter 4}

\section{Concentration and forward prices}

\subsection{Literature and motivation}

This chapter develops a new hybrid model and analyzes the implications of spot market structure for forward prices. In particular, we derive a closed form solution that relates spot market concentration and forward prices. We apply our framework to the highly vertically integrated New Zealand electricity market (NZEM).

As discussed in the introduction, electricity is a non-storable commodity for which spot prices are characterized by the presence of strong seasonal patterns and short-lived trend deviations (spikes). Several papers start from these premises and take into account a broad array of stochastic processes to mimic this observed price behavior. They mostly rely on assumed storage possibilities and make use of no-arbitrage arguments to value derivatives. Schwartz (1997), Schwartz and Smith (2000) and Lucia and Schwartz (2002) concentrate on mean reverting behavior, long-term uncertainty and seasonality. On the other hand, Deng (2000) and Cartea and Villaplana (2005) focus on short-lived oscillations such as jump and spike features. However, these papers frequently rely on estimating nonobservable state variables which is costly in terms of data quality and availability. Few equilibrium insights can be drawn from either of these 
models.

To overcome these disadvantages, a growing literature applies the socalled hybrid models to price derivatives. Hybrid models are composed of two basic stages. First they build on an equilibrium framework when explaining electricity price behavior in terms of observable state variables of demand and supply. Second, they assume a dynamic behavior for state variables and apply no-arbitrage methodologies to price derivatives. This approach offers economic insights into derivative pricing. In other words, derivatives are put in terms of demand and supply parameters.

Skantze, Gubina, and Ilić (2000), Barlow (2002), Pirrong and Jermakyan (2008), Cartea and Villaplana (2008) and Lyle and Elliott (2009) are representatives of this line of research. All these models are characterized by imposing a functional form, based on equilibrium assumptions, for the relationship between price and variables related to demand and supply. Barlow (2002) considers the existence of deterministic and strongly increasing marginal production costs and a stochastic aggregate demand. Skantze et al. (2000) consider the spot price as an exponential function of load and supply bid shifts, treated as stochastic and calculated through principal component analysis. Pirrong and Jermakyan (2008) also propose to model the equilibrium price as a function of two state variables. The state variables are given by electricity demand and the futures price of the marginal fuel, where electricity prices are an increasing function of demand. Cartea and Villaplana (2008) use an exponential function of two observable state variables: demand and generation capacity. They assume that electricity prices are increasing in demand and decreasing in capacity and propose a closed-form pricing model for forward prices taking into account seasonality and heteroskedasticity. Lyle and Elliott (2009) build on Cartea and Villaplana's model and use more sophisticated supply assumptions. They also improve the estimation procedures and derive a closed form solution for European option prices written on average spot prices.

All the aforementioned models implicitly assume competitive markets 
and a pay-as-bid pricing mechanism without explaining if it is a good approximation for markets with more complex structures. None of these derivative models address central aspects of many wholesale electricity markets: market power, vertical integration, contracts and a uniform price auction design. This chapter extends this literature by addressing how more realistic market structure, such as assumed by model I of section 3.3, can affect hybrid pricing modeling.

In particular, equations (3.7) and (3.11) are respectively used in sections 4.2 and 4.3 to analyze forward pricing. We show that spot market concentration and forward prices are closely connected.

\subsection{Model and Results}

A large portion of the energy traded in most competitive electricity markets is hedged. Forward and futures contracts frequently constitute the most significant hedging instruments. This section provides a closed-form solution to evaluate how concentration in the electricity generation industry impacts the forward price curve. Our hybrid pricing model also innovates by taking into account common features of electricity markets such as oligopoly, forward contracts, vertical integration and a uniform price auction mechanism. ${ }^{1}$

Suppose we have two relevant state variables in the market: the aggregate demand and the generators' marginal cost shifter. We assume that for hydro-dominated markets like New Zealand, a good proxy for the cost shifter is the shadow price of water. Increases in this price represent changes in the scarcity of water in the reservoir and affect firms' marginal costs positively. Aggregate demand follows a stochastic process mean reverting towards a deterministic function of time. This function

\footnotetext{
${ }^{1}$ The effect of hedge contracts on spot market power has been the subject of a considerable and influential literature (see section 1.2). Here we address the problem in the opposite direction. We analyze how an increase in the spot market concentration can increase prices in the hedge market.
} 
can be used to describe, for example, seasonal patterns. The shadow price of water follows a simple arithmetic Brownian motion. Interest rates are assumed constant in what follows. Under this assumption, forward and future prices are equal. Formally, we have the following spot market setting:

$$
\begin{aligned}
D_{t}\left(p_{t}^{R}, \vec{W}_{t}\right) & =w_{1 t} \\
M C_{i t}\left(S_{i t}, \vec{W}_{t}\right) & =a+b S_{i t}+\rho w_{2 t} \quad \forall i=1,2, \ldots, K
\end{aligned}
$$

Demand is fully explained by the state variable $w_{1 t}$. State variable $w_{2 t}$ represents the shadow price of water. The parameter $\rho$ reflects how sensitive to changes in $w_{2 t}$ the marginal cost is. In the notation of proposition 3.3.3, we have $c=\kappa_{o}=0$ (perfectly inelastic demand) and $\kappa_{1}=1$. Define $M=\sum_{i=1}^{K} m_{i}$ and assume that the aggregate net position of generators and gentailers is approximately constant $\left(Q C=\sum_{i=1}^{K} Q C_{i t}^{*} \forall t\right)$. Then, by rearranging equation (3.7), the spot price formation equation becomes:

$$
p_{t}=a-\frac{b Q C}{K(K-2)}+\frac{b(K-1-M)}{K(K-2)} w_{1 t}+\rho w_{2 t}
$$

Regarding the state variable dynamics, we assume:

$$
\begin{aligned}
w_{1 t} & =f(t)+x_{1 t} \\
d x_{1 t} & =-\psi x_{1 t} d t+\sigma_{1} d Z_{1} \\
d w_{2 t} & =\mu d t+\sigma_{2} d Z_{2} \\
d Z_{1} d Z_{2} & =\phi d t
\end{aligned}
$$

The aggregate demand $w_{1 t}$ has two components. The first is a completely predictable function of time $f(t)$ which can incorporate seasonality. The second is a diffusion stochastic process $\left(x_{1 t}\right)$. Particularly, $x_{1 t}$ follows a stationary mean-reverting process, or Ornstein-Uhlenbeck process, with a zero long-run mean where the speed of adjustment is $\psi>0$, the volatility 
is $\sigma_{1}$, and $d Z_{1}$ represents an increment to a standard Brownian motion. The shadow price of water $w_{2 t}$ follows an arithmetic Brownian motion with drift $\mu$ and volatility $\sigma_{2}\left(Z_{2}\right.$ is a standard Brownian motion). The state variables are correlated through equation (4.7). The correlation between $Z_{1}$ and $Z_{2}$ is given by $\phi$. The idea is to keep the model simple to infer how the market parameters affect forward prices in an arbitrage pricing setup.

Proposition 4.2.1. Assume the spot price stochastic behavior described by equations (4.3-4.7). We have the following formula for the forward prices PC at $t$ for electricity delivered at time $T$ :

$$
\begin{aligned}
P C\left(p_{t}, T\right)= & a-\frac{b Q C}{K(K-2)}+\frac{b(K-1-M)}{K(K-2)}\left(f(T)+e^{-\frac{\psi b(K-1-M)}{K(K-2)}(T-t)} x_{1 t}\right) \\
& +\rho w_{2 t}+\left(1-e^{-\frac{\psi b(K-1-M)}{K(K-2)}(T-t)}\right) \eta^{*}+\mu^{*}(T-t) \\
\eta^{*}= & -\lambda_{1} \sigma_{1} / \psi \\
\mu^{*}= & \rho\left(\mu-\lambda_{2} \sigma_{2}\right)
\end{aligned}
$$

Where $\lambda_{1}$ and $\lambda_{2}$ are the market prices of risk for demand and for the shadow price of water respectively. Proof: Lucia and Schwartz (2002).

Equations (4.8) explain how $P C$ is affected by the parameters associated with the spot price formation and the state variables in this closed formula.

Corollary 4.2.2. Assume $a \geq 0, b \geq 0, \rho \geq 0, Q C \geq 0, \mu \geq 0, \psi \geq 0$ and $f(T) \geq 0 \forall T$. From equation (4.8) we have the following: $\frac{\partial P C}{\partial a} \geq 0, \frac{\partial P C}{\partial x_{1 t}} \geq 0$, $\frac{\partial P C}{\partial w_{2 t}} \geq 0, \frac{\partial P C}{\partial \mu} \geq 0, \frac{\partial P C}{\partial \sigma_{1}} \leq 0, \frac{\partial P C}{\partial \lambda_{1}} \leq 0, \frac{\partial P C}{\partial \sigma_{2}} \leq 0, \frac{\partial P C}{\partial \lambda_{2}} \leq 0$ and $\frac{\partial P C}{\partial Q C} \leq 0$. Proof: appendix $\mathrm{C}$.

Under the assumptions of Corollary 4.2.2 several results arise. Increases in the fixed portion of the marginal cost $(a)$ affect forward prices positively. Ignoring seasonality issues given by $f(T)$, increases in the current level of demand $\left(x_{1 t}\right)$ and shadow price of water $\left(w_{2 t}\right)$ also increase forward 
prices. Last, ceteris paribus, increases in the exogenous aggregate quantity contracted by generators $Q C$ decreases forward prices.

Regarding dynamics, raising the water price long-term drift $\mu$ augments $P C$. Positive shifts in demand risk $\left(\sigma_{1}\right)$ and/or price of risk $\left(\lambda_{1}\right)$ as well as in water risk $\left(\sigma_{2}\right)$ and/or price of risk $\left(\lambda_{2}\right)$ shift forward prices downwards. That is, an increase in both market prices of risk and cost and demand volatilities decreases forward prices. If the uncertainty is high or expensive, generators accept a smaller price for the same amount of electricity delivered in the future.

On the other hand, increases in the speed of aggregate demand's mean reversion $(\psi)$, in the sensitivity of marginal costs to the shadow price of water $(\rho)$ or in the maturity $(T)$ have an ambiguous effect on $P C$. The impact of $K$ and $b$ on forward prices is also ambiguous. For example, a decrease in $K$ (increase in $b$ ) magnifies the negative effect of the outstanding quantity contracted on forward prices at the same time that it accentuates the positive impact of the demand. The net effect depends on the relationship between variables and parameters such as $K, \psi, b, T, x_{1 t}$, $f(T), M$ and $Q C$. We use arbitrary parameters to show, through an illustrative example, that market power in the spot market and forward prices are possibly connected. Assuming $f(T)=0$ and considering arbitrary parameters, Figure 4.1 illustrates the relationship between the number of generators/gentailers in the market and forward prices for different maturities. In particular, to stress the relationship between $K$, market power and forward prices, this example first considers an electricity market with $Q C \approx 0$. That is, an electricity market where most of the forward contracts are cleared by generators and gentailers. Recall that a gentailers can be net retailers with a long position in the forward market $Q C_{i}<0$.

In this case, an increase in $K$ has two impacts on forward prices. It decreases the equilibrium spot price $p^{c}$ through a decrease in both the average marginal cost $\overline{M C}$ and the average price mark-up $\left(p^{c}-\overline{M C}\right)$. The first effect is directly related to the assumption of decreasing returns to scale 
Figure 4.1: Market power and forward prices as a function of contract maturity (parameters: $a=5, b=0.4, \rho=0.1, \psi=0.8, \sigma_{1}=10, \lambda_{1}=0.5$, $x_{1 t}=50, M=0.5, Q C=0, \mu=20, \sigma_{2}=5, \lambda_{2}=0.5$ and $w_{2 t}=15$ )

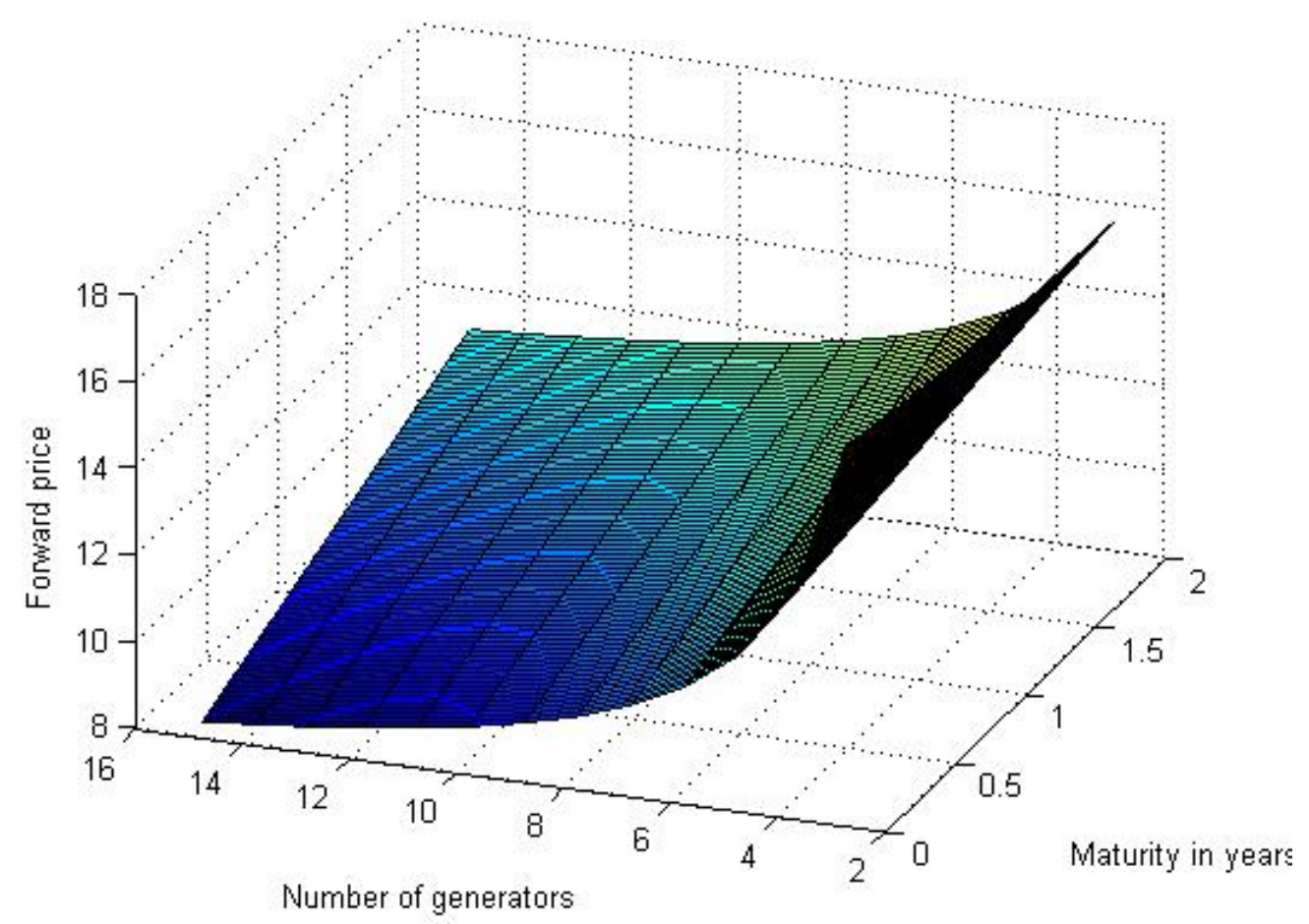

given by the linear marginal cost function with $b>0$. That is, an increase in $K$ decreases the average scale of generators $\left(\frac{D}{K}\right)$, which decreases their average marginal cost (increases their average efficiency). The second effect is related to the average market power exerted by the generators since it affects the average Lerner index. ${ }^{2}$ We observe from Figure 4.1 that positive changes in the number of generators $(K)$, which on average imply a more competitive environment and a more efficient production, reduce forward prices. In fact, an increase in $K$ not only shifts the forward curve

\footnotetext{
${ }^{2}$ Recall that, for simplification, we assumed at chapter 3 that the generators' fixed costs are negligible, which means that mark-ups are exclusively related to market power and are not justified by the recovery of high fixed costs.
} 
downwards but can also rotate it. Thus, market concentration can have different implications along the forward curve. Particularly, the illustrative example shows a situation in which concentration plays a bigger role for shorter maturities. ${ }^{3}$

Figure 4.2: Market power and forward prices as a function of contract maturity (same parameters as Figure 4.1 with $b$ adjusting for a fixed marginal cost)

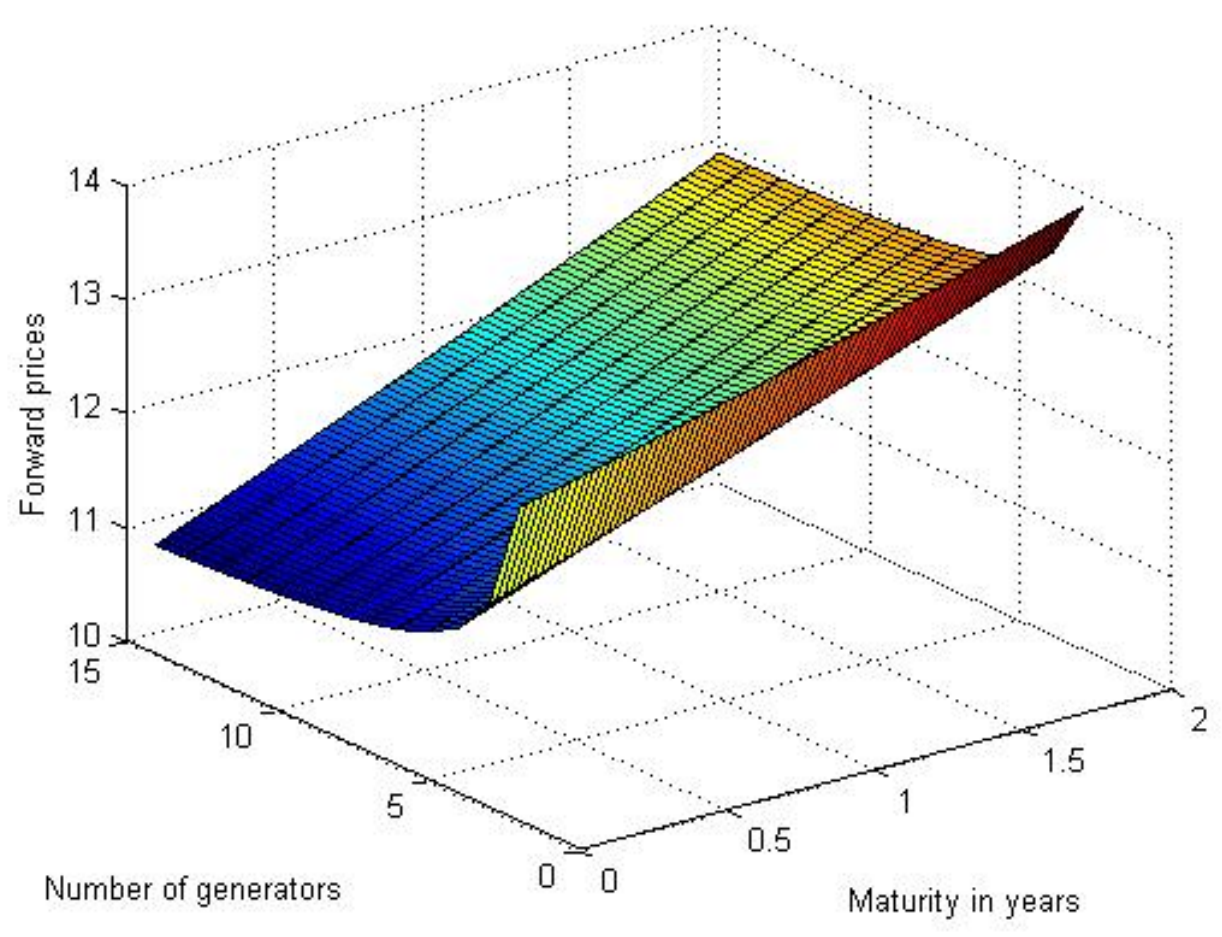

To isolate the market power effect, assume that $b$ adjusts in order to maintain the average marginal cost fixed. Given our assumptions, Figure 4.2 shows that an increase in market power also shifts the forward curve positively and rotates it in the same way as in the previous Figure. How-

\footnotetext{
${ }^{3}$ Given the parameters assumed in Figure 4.1, an increase in $\rho$ or $T$, increases $P C$. For high values of $\sigma_{2}$ (e.g. $\sigma_{2}=200$ ), the effect has the opposite sign. On the other hand, The parameter $\psi$ has a negative effect on $P C$ in the example above. For a sufficient high value of $\sigma_{1}$ (e.g. $\left.\sigma_{1}=1000\right)$, an increase in $\psi$ augments $P C$.
} 
Figure 4.3: Market power and forward prices as a function of contract maturity (Same assumptions as Figure 4.2 except for $Q C=10$ )

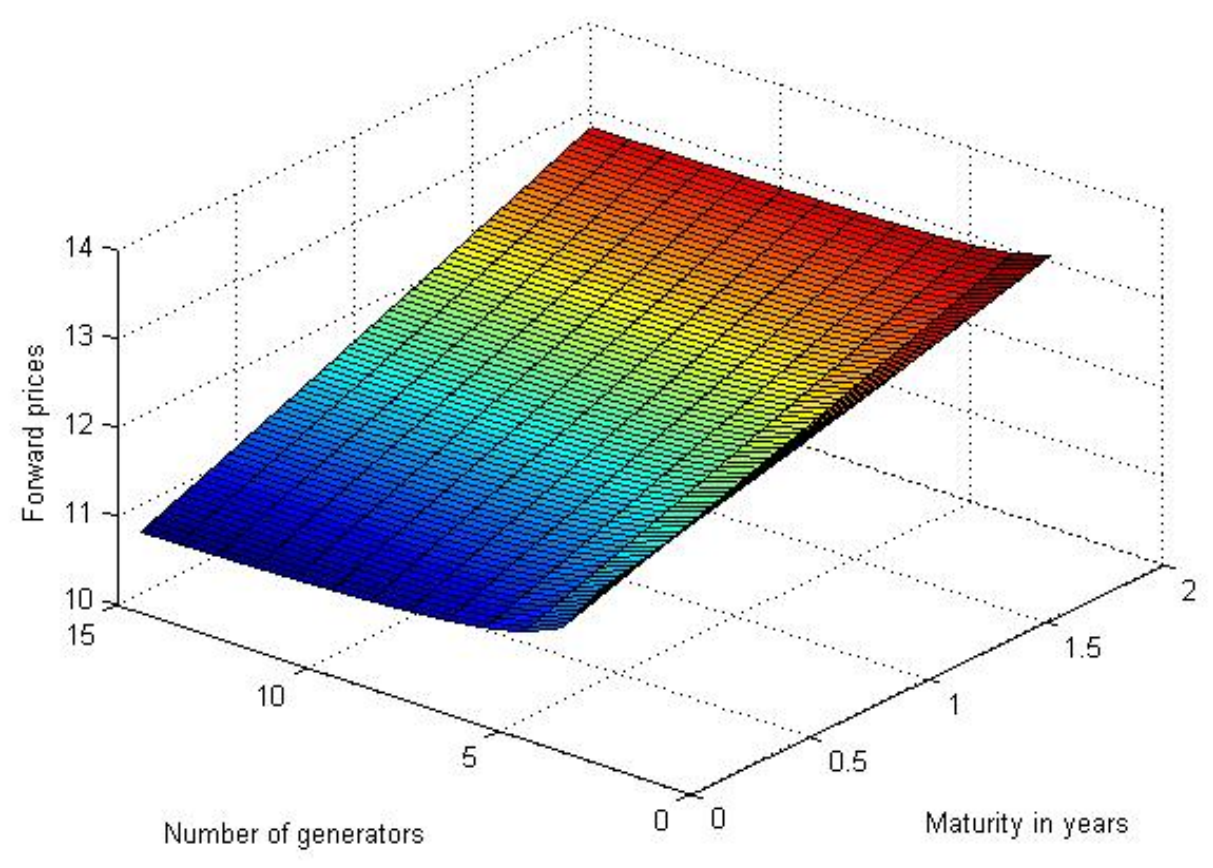

ever, the magnitude of the impact of $K$ on the forward price $P C$ is reduced when controlled for its effect on the average $M C$.

Given our assumptions, the fact that forward prices can be higher in electricity markets with less generators is a particularly relevant result. It means that, contrary to results frequently observed in the literature, there is a possible situation where forward contracts, instead of reducing the spot market power, can be, in fact, affected by it (since $P C$ is potentially affected by market power). For example, Allaz and Villa (1993), Newbery (1998), Green (1999) and Bushnell (2007) observe the importance of existing forward contracts to reduce market power.

Figure 4.3 analyzes the effect of assuming $Q C=10(20 \%$ of the assumed initial demand $x_{1 t}$ ). The other parameters are the same as used in Figure 4.2. It shows that there is still a positive, but smaller, effect of mar- 
ket power on the forward curve. This is reasonable, since an increase in $Q C$ decreases spot price mark-up as shown by equation 3.5.

This exercise shows that if the market becomes less concentrated the forward curve can be shifted or rotated. Specifically, generators' market power in the spot market can inflate forward prices and translate into market power in the hedge market. This analysis has let $K$ change given $Q C$ fixed. In chapter 6 , we consider the determination of the equilibrium $P C$ and $Q C$.

\subsection{Empirical Exercise}

The objective of this section is to use our forward pricing model to analyze the New Zealand Electricity Market (NZEM). We adopt a two-step empirical strategy. The first step consists of estimating the spot price model parameters. The second step involves the implicit calibration of the market prices of risk $(\lambda$ 's) from the observed forward prices.

The reason for not estimating the spot price model and the market prices of risk jointly is that the forward price data is unbalanced and irregular. That is, we have days where several overlapping forward contracts are traded and days with no trade at all. Reconciling the spot price data with the forward price data available would imply losing relevant information about the spot market dynamics. Besides, the forward price formula is non-linear in several spot price model parameters which would unnecessarily complicate the empirical exercise.

The electricity spot market in New Zealand is characterized by a bidbased nodal market where half-hourly uniform-price auctions establish the spot prices for each relevant node of the system. NZEM also has an active forward market and potentially oligopolistic wholesalers. Table 4.1 shows that the NZEM has a concentrated spot market with $K=5$ big players and presents a high degree of vertical integration. That is, the retail market share of generators is equal to $\left.M=\sum_{i=1}^{K} m_{i}=95 \%\right)$. Notice that 
Table 4.1: Market Shares in NZ (2008)

\begin{tabular}{lcc}
\hline Company & Generation & Retail \\
\hline Contact Energy & $26 \%$ & $27 \%$ \\
Genesis Energy & $22 \%$ & $25 \%$ \\
Meridian Energy & $28 \%$ & $12 \%$ \\
Mighty River Power / Mercury Energy & $14 \%$ & $19 \%$ \\
Trust Power & $5 \%$ & $11 \%$ \\
\hline Total & $95 \%$ & $94 \%$ \\
\hline
\end{tabular}

Source: Companies' annual reports 2008 and NZ Electricity Commission.

it does not mean that the gentailers have the same market share in both the generation and retail markets. For example, Meridian Energy is a net generator with $28 \%$ of the generation market share and $12 \%$ of the retail market share and, on the other hand, Mercury Energy is a net retailer with $12 \%$ of the generation market and $19 \%$ of the retail market.

As an approximation, suppose that the assumptions of corollary 3.3.5 hold. That is, we assume that NZEM is fully vertically integrated $(K=N)$. This means that the market is predominantly composed of gentailers, that can be either net retailers such as Mercury Energy or net gentailers such as Meridian Energy. That is, individual firms are not necessarily fully vertically integrated. Under our equilibrium framework, markets with a very high degree of vertical integration (not necessarily of individual firms) have a clearing spot price which is not affected by forward contracts. ${ }^{4}$ Individual gentailers (net generators) in such markets can exert market power. However, by equation (3.5), the average spot price mark-up in equilibrium is equal to zero. ${ }^{5}$ The $K=N$ assumption also offers a simple linear relationship between electricity spot price and state variables, given by equation (3.11), which can easily incorporate dynamics.

The New Zealand electricity market is dominated by hydro power with

\footnotetext{
${ }^{4}$ The determinants of the hedging decision are the subject of chapter 6 .

${ }^{5}$ For all $K>2$, equation (3.5) states that $E(p)-M C=0$ in situations where an individual gentailer is fully vertically integrated. Equation (3.5) also shows that, if gentailers own the entire retail market, the average mark-up equals zero even if individual firms are not fully vertically integrated.
} 
significant participation of gas thermal generation. Therefore, the basic candidates for marginal cost shifters would be the prices of stored water and/or gas. In particular, Evans, Guthrie, and Lu (2010), take into account the optimal intertemporal choices regarding electricity production and water storage and show that, once adjusted for transmission costs, the shadow price of water is the same as the shadow price of gas. ${ }^{6}$ However, shadow prices are by definition non-observable variables. ${ }^{7}$ The challenge is to find the best proxy or proxies for these generation inputs.

There are some primary candidates. The water inflow to the hydro system, for example, is clearly correlated with the shadow price of water. The storage option decreases in value when the inflows are abundant and increases when inflows are scarce. By similar reasons, past gas generation could also be used as a proxy for the shadow prices of stored gas. International gas or oil price indexes could be another possibility. All these alternatives present the same important drawback: they abstract from marginal valuations and are autocorrelated.

We first consider water inflows $\left(\mathrm{m}^{3} / \mathrm{s}\right)$ in the hydro system as the cost shifter $w_{2 t}$. The lagged spot price $p_{t-1}$ is a superior alternative. $p_{t}$ is observable and approximately equal to the marginal cost of gas (and therefore water) adjusted for the spark gap (See Evans and Guthrie (2009)). Thus, $p_{t-1}$, which is not endogenous, might well approximate the shortrun marginal cost of the generators.

We use daily frequency data from 22/01/2004 to 30/11/2010. The daily frequency is consistent with approximating the continuous time assumption of our spot price model. The adopted data range corresponds to the maximum interval of negotiated forward contracts that we obtained for the New Zealand electricity market (NZEM).

The Haywards node spot price is assumed to be a proxy for the na-

\footnotetext{
${ }^{6}$ According to the same authors, the exceptions correspond to the rare situations where lakes are entirely full.

${ }^{7}$ While obviously the case for hydro it also holds for gas in the absence of a spot market and limitations of gas supply. See Evans and Guthrie (2009).
} 
tional spot price $p_{t}$. The demand variable $w_{1 t}$ is defined as the NZ national daily offtake (in Gwh). We analyze two variables as the cost shifter $w_{2 t}$ : the water inflow to the NZ hydro system and the lagged spot price $p_{t-1}$. The source for all these variables is the New Zealand Electricity Commission. ${ }^{8}$ The forward prices are extracted from the negotiated Haywards monthly and quarterly forward contracts. As mentioned before, the data is irregular and unbalanced since there are days with no trade and overlapping contracts of different maturity or nature. The source for the forward prices is the EnergyHedge website. ${ }^{9}$ Both the spot prices and the forward prices are adjusted for the New Zealand Consumer Price Index (CPI).

\subsubsection{First step}

The empirical estimation of the spot market parameters requires the discretization of the continuous equations (4.4-4.7), which yields the following equations:

$$
\begin{aligned}
& x_{1 \hat{t}}=w_{1 \hat{t}}-f(\hat{t}) \\
& x_{1 \hat{t}}=\left(1-\frac{\hat{\psi}}{365}\right) x_{1 \hat{t}-1}+\epsilon_{1 \hat{t}} \\
& w_{2 \hat{t}}=w_{2 \hat{t}-1}+\frac{\hat{\mu}}{365}+\epsilon_{2 \hat{t}}
\end{aligned}
$$

Define $\hat{t}$ as a discrete period of time (In our case, a day). Following the approach of Lucia and Schwartz (2002), we define the deterministic com-

\footnotetext{
${ }^{8}$ The data was specifically extracted from the centralised dataset (CDS) available at http://www.ea.govt.nz/industry/modelling/cds/ (accessed on 20/06/2011). There is no daily aggregate data of water inflow available. The daily water inflow to NZ $\left(w_{2 t}\right)$ was built from the sum of the daily inflows to the hydro systems described by Table 1 of Harte, Pickup, and Thomson (2004).

${ }^{9}$ The data was collected at http:/ / www.energyhedge.co.nz (accessed on 30/01/2011). The website is not currently available since the EnergyHedge company signed an agreement with the Australian Stock Exchange (ASX) at 03/06/2011.
} 
ponent of the demand variable as the following cosine function:

$$
f(\hat{t})=\zeta+v \cos \left(\frac{\hat{t}}{365}+\tau\right)
$$

The advantage of this approach over the use, for example, of dummy variables to model seasonality is that $f(\hat{t})$ is continuous and easily integrable. Most forward contracts in New Zealand involve the delivery in a specific month or quarter. The integrability of $f(\hat{t})$ allows for a closed-form solution for the forward price of these average periods. Later, we show that this definition of $f(\hat{t})$ fits the demand behavior quite closely. Taking into account $f(\hat{t})$ and the equations (4.9-4.11), the spot price formation is defined by the following system of equations:

$$
\begin{aligned}
p_{\hat{t}} & =a+\frac{b}{K} w_{1 \hat{t}}+\rho w_{2 \hat{t}}+\epsilon_{\hat{t}} \\
w_{1 \hat{t}} & =f(\hat{t})+\left(1-\frac{\hat{\psi}}{365}\right)\left(w_{1 \hat{t}-1}-f(\hat{t}-1)\right)+\epsilon_{1 \hat{t}} \\
w_{2 \hat{t}} & =w_{2 \hat{t}-1}+\frac{\hat{\mu}}{365}+\epsilon_{2 \hat{t}} \\
f(\hat{t}) & =\zeta+v \cos \left(\frac{\hat{t}}{365}+\tau\right)
\end{aligned}
$$

The spot price model parameters are estimated by the seemingly unrelated regression method (SUR), where $\epsilon_{\hat{t}}, \epsilon_{1 \hat{t}}$ and $\epsilon_{2 \hat{t}}$ are assumed to be independent, to have zero mean and to have a finite covariance matrix. ${ }^{10}$ That is, we assume that the right hand side variables of the system are all exogenous. This is consistent with the theoretical assumptions of the model since $\vec{W}$ is by definition exogenous. Thus, we are strictly concerned about the estimation of the conditional expectations of equations (4.12-4.15). Define $w_{2 \hat{t}}$ as the NZ aggregate water inflow as previously explained. The estimation results are given by Table 4.2. Notice that both

\footnotetext{
${ }^{10}$ See Greene and Zhang (2003) chapter 14.
} 
Table 4.2: Estimation Results: $w_{2 \hat{t}}=$ water Inflows. Equations (4.12-4.15). Data: NZ Electricity Commission

Method: SUR

Sample: 22/01/2004 30/11/2010

Included observations: 2505

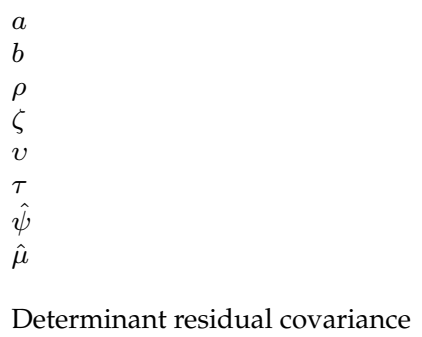

Coefficient Std. Error

t-Statistic Prob.

$\begin{array}{ll}-16.15 & 12.91 \\ 5.66 & 0.60 \\ -1.79 \mathrm{E}-05 & 1.52 \mathrm{E}-06 \\ 102.98 & 0.28 \\ -8.68 & 0.40 \\ 4.95 & 0.01 \\ 148.87 & 5.81 \\ -234,501 & 4,339,872\end{array}$

$-1.25 \quad 0.21$

$9.40 \quad 0.00$

$-11.77 \quad 0.00$

$365.00 \quad 0.00$

$\begin{array}{ll}-21.78 & 0.00\end{array}$

$677.56 \quad 0.00$

$25.64 \quad 0.00$

$3.15 \mathrm{E}+16$

$p_{\hat{t}}=a+\frac{b}{K} * w_{1 \hat{t}}+\rho * w_{2 \hat{t}}$

R-square

Adjusted R-square

S.E. of regression

Durbin-Watson stat

$w_{1 \hat{t}}=\zeta+v * \cos \left(2\left(\frac{\hat{t}}{365}+\tau\right) \pi\right)$

$+\left(1-\frac{\hat{\psi}}{365}\right)\left(w_{1 \hat{t}-1}-\left(\zeta+v * \cos \left(2\left(\frac{\hat{t}-1}{365}+\tau\right) \pi\right)\right)\right)$

R-square

Adjusted R-square

S.E. of regression

Durbin-Watson stat

$w_{2 \hat{t}}=w_{2 \hat{t}-1}+\frac{\hat{\mu}}{365}$
0.62

0.62

5.82

1.54
Mean dependent var 102.98

S.D. dependent var $\quad 9.48$

Sum squared resid $\quad 86,591.75$

$0.085 \quad$ Mean dependent var $\quad 74.68$

$0.084 \quad$ S.D. dependent var $\quad 57.72$

Sum squared resid

7059760

R-square

Adjusted R-square

S.E. of regression

Durbin-Watson stat

$\begin{array}{lll}0.40 & \text { Mean dependent var } & 1,424,762 \\ 0.42 & \text { S.D. dependent var } & 748,967 \\ 579,353.3 & \text { Sum squared resid } & 7.96 \mathrm{E}+14 \\ 2.17 & & \end{array}$


the demand and the water inflows are statistically significant. Their parameters also present the expected signs. Positive shifts in the demand and negative changes in the water inflows increase the equilibrium spot price $(b>0$ and $\rho<0)$. The remaining results show that the aggregate demand is reasonably (and significantly) explained by the deterministic function $f(\hat{t})$. As the magnitude and significance of $\hat{\psi}$ suggests, the demand reverts quickly to $f(\hat{t})$. Figure 4.4 illustrates this result. On the other

Figure 4.4: Demand - NZEM Offtake (Gwh). Function $\mathrm{f}(\mathrm{t})$ given by equation (4.15): $\hat{\zeta}=102.98, \hat{v}=-8.68$ and $\hat{\tau}=4.95$. Data: NZ electricity Commission.

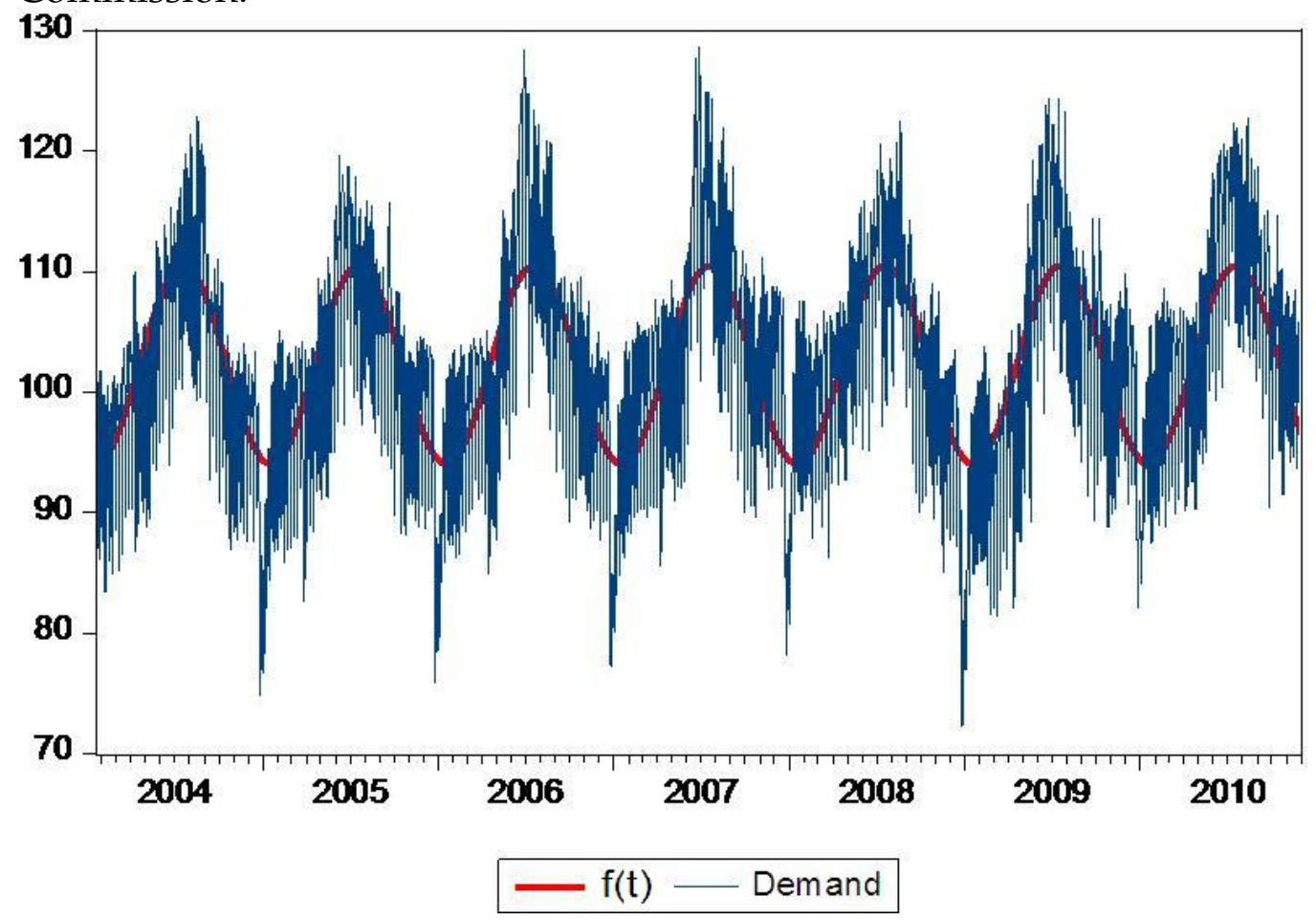

hand, the water inflows' drift $\hat{\mu}$ is not statistically different from zero. This is not surprising since inflows are stationary. Besides, the $R^{2}$ of the spot price equation is almost insignificant $(<10 \%)$ with a serious autocorrelation problem, expressed by a Durbin-Watson (DW) statistic very different from 2. This (and the significance of the autoregressive component $p_{\hat{t}-1}$ 
expressed in Table 4.3) indicates that the hypothesis of $\operatorname{cov}\left(\epsilon_{\hat{t}}, \epsilon_{\hat{t}-1}\right)=0$ does not hold. This is evidence that the water inflows $w_{2 \hat{t}}$ alone do not satisfactorily capture the generators' marginal cost behavior. Several combinations of $w_{2 \hat{t}}$ and other related variables such as water storage and gas generation were tried. All failed to attain serially uncorrelated results. ${ }^{11}$

In fact, as shown by Lucia and Schwartz (2002) and Mason (2002), $p_{\hat{t}}$ presents a strong autoregressive component. In our second approach, we assume that the observed price is a good best proxy available for capturing shifts in the marginal costs. Specifically, we assume for the reason given earlier, the lagged price $p_{\hat{t}-1}$ is a good empirical proxy for $w_{2 \hat{t}}$. Including $p_{\hat{t}-1}$ in the regression increases the goodness-of-fit given by the $R^{2}$, and it considerably attenuates the serial correlation problem (see Table 4.3). All the remaining parameter estimates of Table 4.3 yield results similar to the previous exercise using the other proxies for marginal cost. The fast mean reversion of the aggregate demand to a significant deterministic function remains. Again, the drift $\hat{\mu}$ is insignificant.

The lagged spot price alone does not exhaust all the time series possibilities of the daily electricity spot price in the NZEM market. A purely empirical seasonal autoregressive integrated moving average (SARIMA) approach would suggest the additional consideration of moving average and autoregressive components. ${ }^{12}$ However, the object of this subsection is to test and estimate our spot price model taking into account the NZEM data and the results of Table 4.3 show that our model fits well the actual NZEM data. First, all the parameters are statistically significant, with the exception of the drift $\hat{\mu}$ which is not expected to be different from zero. Second, the explanatory power of the equilibrium spot price equation is reasonable $\left(R^{2} \approx 75 \%\right)$. Last, the serial correlation problem is attenuated with the adoption of $p_{\hat{t}-1}$ as a proxy to $w_{2 \hat{t}}$.

In summary, this second approach fits closely the actual NZEM data

\footnotetext{
${ }^{11}$ Serial correlation affects the quality of estimates and mean that the best forecast of $p_{\hat{t}}$ is not the estimated equation.

${ }^{12}$ See Enders (1995) for an introductory discussion about SARIMA models.
} 
Table 4.3: Estimation Results: $w_{2 \hat{t}}=p_{\hat{t}-1}=$ lagged spot price. Equations (4.12-4.15). Data: NZ Electricity Commission

Method: SUR

Sample: 22/01/2004 30/11/2010

Included observations: 2505

Coefficient Std. Error t-Statistic Prob.

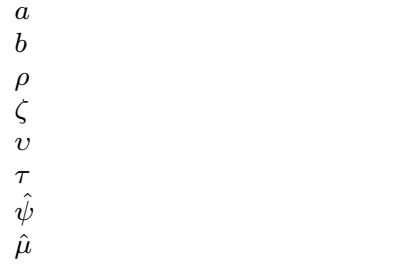

$-14.83 \quad 1.75$

$\begin{array}{lll}1.75 & -8.46 & 0.00 \\ 0.08 & 9.29 & 0.00\end{array}$

$\begin{array}{llll}0.76 & 0.08 & 9.29 & 0.00\end{array}$

$\begin{array}{llll}0.99 & 0.003 & 368.79 & 0.00\end{array}$

$\begin{array}{llll}102.97 & 0.29 & 358.65 & 0.00\end{array}$

$\begin{array}{llll}-8.23 & 0.39 & -21.15 & 0.00\end{array}$

$\begin{array}{llll}4.96 & 0.01 & 674.03 & 0.00\end{array}$

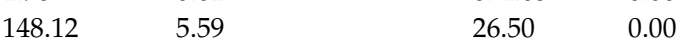

$\begin{array}{llll}2.89 & 206.28 & 0.01 & 0.99\end{array}$

Determinant residual covariance

$3.15 \mathrm{E}+16$

$p_{\hat{t}}=a+\frac{b}{K} * w_{1 \hat{t}}+\rho * p_{\hat{t}-1}$

R-square

Adjusted R-square

S.E. of regression

0.76

0.76

28.04

Durbin-Watson stat

$w_{1 \hat{t}}=\zeta+v * \cos \left(2\left(\frac{\hat{t}}{365}+\tau\right) \pi\right)$

$+\left(1-\frac{\hat{\psi}}{365}\right)\left(w_{1 \hat{t}-1}-\left(\zeta+v * \cos \left(2\left(\frac{\hat{t}-1}{365}+\tau\right) \pi\right)\right)\right)$

R-square

Mean dependent var $\quad 103.12$

Adjusted R-square

S.E. of regression

$\Delta p_{\hat{t}}=\frac{\hat{\mu}}{365}$

Adjusted R-square 
between 22/01/2004 and 30/11/2010. The equilibrium and dynamic parameters are statistically significant with the expected signs. The second step in the forward price modeling is the calibration of the market prices of risk $\left(\lambda_{1}\right.$ and $\left.\lambda_{2}\right)$ from the actual forward price data.

\subsubsection{Second step}

The next step in the implementation of our model involves calibrating the demand market price of risk $\left(\lambda_{1}\right)$ and the supply market price of risk $\left(\lambda_{2}\right)$. We use the non linear least squares (LS) approach. That is, we choose the $\lambda$ 's that minimize the sums of the squares of deviation between the observed forward prices and the theoretical formula given by equation (4.16). Notice that equation (4.8) refers to the forward price at $t$ of delivering electricity at the future instant $T$. However, electricity contracts are not instantaneous. They entail a specific time interval. The Haywards forward contracts used in our exercise refer to monthly and quarterly periods of time. That is, the observed forward price $\hat{P C_{t}}$ refers to the forward price cleared at $t$ of a fixed flow of electricity to be delivered between $T_{1}$ and $T_{2}$, with $T_{2}-T_{1}$ being a month or a quarter. Therefore instead of directly using equation (4.8) for pricing, we use its integral between the maturities $T_{1}$ and $T_{2}$. That is, we use the following equation:

$$
\overline{P C}_{t}^{T_{1}, T_{2}}=\int_{T_{1}}^{T_{2}} P C\left(t, T, w_{1 t}, w_{2 t}, \hat{\theta}, \hat{\lambda_{1}}, \hat{\lambda_{2}}\right) d T
$$

Where $\hat{\theta}$ is a vector that comprises all the estimated parameters of Table 4.3 , including the standard deviations: we consider the conditional standard deviations of demand $s_{1}$ and spot price $s_{2}$ as proxies for volatility in our calibration exercise. In addition, we adjust these daily volatilities for the annual basis in which the dynamic processes are defined $(t$ is proportional to a year). That is, we use $\hat{\sigma}_{1}=s_{1} \sqrt{365}=5.84 \sqrt{365}$ and $\hat{\sigma}_{2}=s_{2} \sqrt{365}=28.29 \sqrt{365}$. The calibrated market prices of risk for 
the Haywards contracts between 22/01/2004 and 30/11/2010 are then $\hat{\lambda}_{1}=3.63$ and $\hat{\lambda}_{2}=0.005$. Now, we have all the elements for constructing an estimated forward price curve. Consider a monthly contract. For illus-

Figure 4.5: Market concentration and forward prices in the New Zealand Electricity Market (NZEM). Equations (4.12-4.15). Parameters: Table 4.3. Data: NZ Electricity Commission

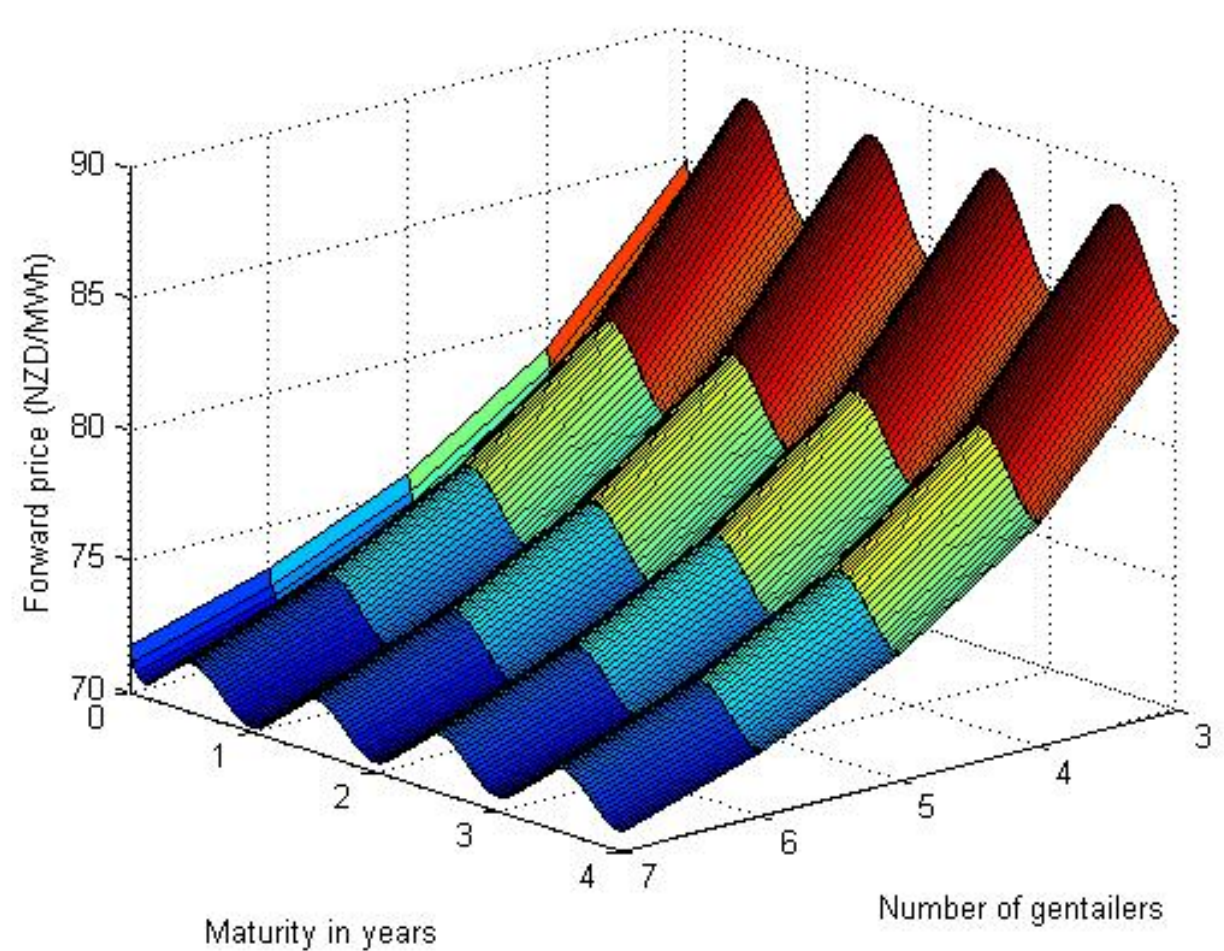

trative purposes, assume that $t=0$ (We price the forward contracts at the first day of the year) and that the demand and cost shifter are, respectively, estimated by the sample means $\hat{E}\left(w_{1}\right)=103.12$ and $\hat{E}\left(w_{2}\right)=\hat{E}(p)=72.66$. Figure 4.5 shows that spot market concentration in fact affects forward prices in the NZEM market. As expected, an increase in the number of firms decreases forward prices significantly. For example, the peak $P C$ varies from around NZD70.00, in a market with 7 firms, to more than NZD85.00, in a market with just 3 firms (increase of more than $20 \%$ ). We 
observe that the NZEM calibrated forward curve is essentially flat. The demand seasonal pattern clearly dictates the forward curve shape.

\subsection{Summary}

We consider the existence of two state variables: the aggregate demand and the shadow price of fuel (water and gas) moving marginal cost functions. Assuming similar stochastic processes to Lucia and Schwartz (2002) and applying their two factor arbitrage model results over our equilibrium spot price formation rule (equation (3.7)), we derive a closed form forward price (equation (4.8)). Several results can be derived from comparative statics. Increases in either volatility or the price of risk of both demand and shadow price of fuel decrease the price of forward contracts. The opposite happens when raising the fuel shadow price's drift or the current value of both state variables. Positive shifts in the generators' cost functions or in the deterministic portion of aggregate demand also move forward prices upwards.

We use an illustrative numerical example (Figure 4.1) to show that a decrease in the number of generators can shift the forward curve positively. In particular, controlling for the efficiency effect of an increase in concentration, Figure 4.2 shows that market power in the spot market can be transferred to the forward market.

Section 3.3 shows that there are two market structures where the spot price is not influenced by firms' forward positions. The first one is the case of perfectly competitive markets. The second corresponds to electricity markets where all the retailers are actually gentailers $(K=N)$. In this situation, the forward net positions of all the retailers would be reflected in the gentailers' optimal supply strategies. If the participation of outside speculators in the forward market is negligible, as we assumed, the impact of forward contracts on the aggregate supply curve is completely offset when all the generators' (gentailers included) individual supplies are 
aggregated. This case can be thought of as an approximation for markets with a high degree of vertical integration as is the case in New Zealand or Spain. Notice that this assumption allows for differences across gentailers in their participation in wholesale and retail market. That is, the market is allowed to be composed by net retailers and net generators. This assumption also means that, despite the average Lerner index in such fully vertically integrated markets being equal to zero according to our model, individual net gentailers exert spot market power.

We assume $K=N$ as a good approximation for the New Zealand electricity market (see Table 4.1) and apply a two-stage empirical methodology implementing the model for the NZEM market. Our model fits the data reasonably and confirms the relevance of spot market concentration in affecting forward prices.

In summary, chapter 4 develops the link between market structure and forward prices. We show that if the market becomes less concentrated the forward curve can be shifted or rotated. Specifically, generators' market power in the spot market can inflate forward prices and translate into market power in the hedge market. 


\section{Chapter 5}

\section{Investing in vertical integration}

\subsection{Motivation}

The purpose of this chapter is to model the relationship between vertical integration and market structure in electricity markets. We adopt the same hybrid approach of the last chapter to assess how changes in initial market parameters affect the generators' decision to increase their participation in the electricity retail market. In other words, we analyze how changes in parameters such as $\sigma_{1}$ (demand volatility), $K$ (measure of spot market power) and $M=\sum_{i=1}^{K} m_{i}$ (initial degree of vertical integration) affect the value of a retail expansion project (vertical integration) and the timing of this investment based on demand. In particular, this chapter analyzes the case of short-term projects to temporarily increase the vertical integration of a specific gentailer. ${ }^{1}$

The determinants of vertical integration (VI) are a subject of intense debate. Section 3.3 offers a brief discussion about possible causes and consequences suggested by the literature. This chapter analyzes vertical integration from an investment perspective. In particular, we propose a

\footnotetext{
${ }^{1}$ The framework here developed can also be used to analyze long-term vertical integration projects at the cost of modeling additional sources of uncertainty and allowing for retail price adjustments.
} 
hybrid equilibrium/real options framework to model the vertical integration decision.

The cost structure of the electricity retailers has several components. Retail businesses can be divided into two distinct groups: the low voltage regional distribution (lines business) and the commercialization of energy (energy business). On one hand, retailers operate and maintain copper and/or aluminium wires, power poles and connection points. ${ }^{2}$ On the other hand, retailers undertake community/social initiatives and marketing campaigns in order to sell more electricity and strengthen their reputation. They also invest in call centres for account and fault management. For simplification, we assume in our equilibrium model (chapter 3 ) that the marginal costs associated with both the distribution O\&M expenditures and selling expenses are negligible in comparison to the marginal costs of power generation.

Gentailers can raise their retail market share by either taking consumers from rival gentailers or from pure retailers. A gentailer's decision to increase his vertical integration in the short-term (say, 4 years), involves undertaking projects for either expanding his regional distribution coverage or for increasing his marketing expenses. A considerable proportion of the costs associated with marketing campaigns and the expansion of lines coverage is irreversible. In particular, advertising in marketing campaigns entail totally sunk expenditure. It may also include a situation of a retailer entering a region as starting up, incurring transactions costs that are sunk.

As discussed in the previous chapters, electricity markets are characterized by volatile spot prices and uncertainty about the demand. Both aspects directly affect the retail business. In an uncertain scenario with sunk costs, the value of flexibility is relevant to the decision of a generator to expand his participation in the retail market. In other words, the option to wait for undertaking the project of increasing his vertical integration

\footnotetext{
${ }^{2}$ In New Zealand, lines companies do this job and retailers contract for delivery service over lines.
} 
has a positive value.

Precisely, we consider the specific issue of a gentailer's decision to irreversibly invest in a marketing campaign (and/or in an expansion of the distribution coverage) to increase his retail market share. For simplification, we assume that $i$ ) the effect of this campaign is instantaneous and has a pre-determined and certain duration, ii) the sunk costs associated with such campaign are one shot and instantaneous iii) demand is the only source of uncertainty, iv) the retail price is fixed for the investment horizon, v) competitors do not react strategically to this campaign and vi) forward contracts are given.

The reason for the assumptions i) and ii) is to focus on the determinants of vertical integration, avoiding complications arisen from more practical considerations about the nature of either the investments or the marketing campaign. The assumption iii) of only one state variable (demand) allows for the derivation of a closed-form solution for the problem. The rigidity of retail prices given by iv) is a reasonable approximation for the short and medium-term in markets that do not face abnormal changes in demand. ${ }^{3}$ The assumption v) of no predictable strategic reaction to an increase in a gentailer's vertical integration simplifies the model considerably and enables for a ceteris paribus analysis. ${ }^{4}$ Since the retail price is assumed to be rigid, changes in demand that increase the spot prices have an initial neg-

\footnotetext{
${ }^{3}$ The timing and velocity of the retail price adjustments depend on the nature of the market and the retailers' expectation about future demand. For example, the more elastic is the demand, the greater is the sensitivity of retail prices to changes in the expected demand. The length of contracts also affects how fast changes in expected demand are translated into variations of equilibrium retail prices. Last, retail prices are also frequently subject of price regulation (price cap or rate of return). The more binding are these price constraints, the less retail prices are enabled to vary. A preliminary work allowing for retail prices to react to demand periodically is currently being conducted and achieves similar qualitative results.

${ }^{4}$ The results of this chapter must be viewed as a best case scenario, since the rivals reaction to the gentailer's project of increasing his retail market share would almost certainly decrease the value of his project. The extension of the framework here developed to enable for the possibility of strategic interactions among firms in the market is a promising avenue for future research.
} 
ative impact on the retail profit margin. Lastly, the assumption vi) implies that we take a partial equilibrium approach since forward contracts do not react to increases in vertical integration. That is, this exercise analyzes the value of vertical integration taking a specific $K$-dimensional vector of generators' quantity contracted $\overrightarrow{Q C}$ as given. ${ }^{5}$

\subsection{Model}

Formally, assume a particular electricity market that meets the assumptions of model I of section 3.3. We have $K$ generators in the market and a specific generator/gentailer $i$ is considering to irreversibly invest $I$ to expand his retail market share by $\Delta m_{i}$ for $h$ years. Assume that $\omega \Delta m_{i}$ of this increase is taken from the rival gentailers $j$ (where $j \neq i$ and $j=$ $1,2, \ldots, K-1)$, changing their average retail market share by $\Delta \bar{m}_{j}=$ $-\frac{\omega}{K-1} \Delta m_{i}$. Notice that $\omega \in[0,1]$. Obtaining retail share from pure retailers will have different effects than obtaining retail share from rival generators. As an illustration, we retain an oligopoly in generation $(K=5)$ and assume that a generator has the option to irreversibly invest 100 million dollars in a marketing campaign to increase his retail market share by $2 \%$ for the next 4 years. Assume that $\omega=60 \%$, which implies that $1.2 \%$ of the market share is taken from rival gentailers and $0.8 \%$ is taken from pure retailers. In this case, the average market share of firm $i$ 's rival gentailers would change by $\Delta \bar{m}_{j}=-0.30 \%$.

Lemma 5.2.1. Assume that the assumptions of section 3.3 hold. Assume that demand itself is the only state variable and has no effect on firms' marginal costs $\left(\vec{W}_{t}=D_{t}, \rho=0\right.$ and $\left.\kappa=1\right)$. A ceteris paribus increase at $t$ in generator/gentailer $i$ 's retail market share by $\Delta m_{i}$, accompanied by a decrease of $\omega \Delta m_{i}$ in rival generators' retail market share, implies the following change in his profit

\footnotetext{
${ }^{5}$ It is reasonable to expect that the optimal hedging decreases as a result of an increase in vertical integration. In fact, we demonstrate this result in Chapter 6. In the end of this chapter, we conduct simulations to show how the results are affected by changes in $Q C_{i}$.
} 
level:

$$
\Delta \pi_{i t}=\hat{A} D_{t}-\hat{B} D_{t}^{2}
$$

where,

$$
\begin{aligned}
\hat{A}= & \Delta m_{i}\left(p^{R}-a+\frac{b}{K(K-2)}\left(1-\frac{\omega}{(K-1)^{2}}\right) Q C-\frac{b \omega}{(K-1)^{2}} Q C_{i}\right) \\
\hat{B}= & \frac{b \Delta m_{i}}{K(K-2)}\left((K-1-M)\left(1-\frac{\omega}{(K-1)^{2}}\right)+\frac{\omega K(K-2)}{(K-1)^{2}} m_{i}-\right. \\
& \left.\left(1+\frac{\omega}{(K-1)^{2}}\right) \frac{\Delta m_{i}}{2}\right)
\end{aligned}
$$

Proof: appendix C.

Equation (5.1) shows the impact on profit at time $t$ of a change in firm $i$ 's market share. This equation reflects fixed retail prices for all players in our model and the absence of demand effects except those arising from exogenous shocks. Assuming everything else constant, an increase in demand increases the clearing spot price $p_{t}^{c}$ which decreases the retail margin $\left(p^{R}-p_{t}^{c}\right)$ and increases the amount of electricity sold by gentailer $i$ in the retail market $\left(m_{i} D_{t}\right)$. Therefore, an increase in demand has an ambiguous effect on retail profit. The retail margin can become negative for high demand scenarios so that a marginal increment of the demand can decrease firm $i$ 's profit.

If $\omega$ is equal to zero (i.e firm $i$ expands his retail positions solely out of pure retailers' market shares), the first order effect of changes in gentailer $i^{\prime}$ s retail share on his profit is equal to $\Delta \pi_{i t}=\Delta m_{i}\left(p^{R}-p_{t}^{c}\right) D$. In equilibrium, all the indirect effects of changes in firm $i$ 's market share cancel out. That is, an increase in gentailer $i$ 's retail share $\left(\Delta m_{i}\right)$ causes an increase in his equilibrium supply $S_{i}^{*}$ which in turn increases his marginal costs $M C_{i}$ and reduces the price mark-up $p^{c}-M C_{i}$. As shown in appendix $C$, the overall impact of these indirect effects on firm $i$ 's profit is zero, i.e. $\frac{\partial \pi_{i}}{\partial p^{c}} \frac{\partial p^{c}}{\partial m_{i}}=-\frac{\partial \pi_{i}}{\partial S_{i}^{*}} \frac{\partial S_{i}^{*}}{\partial m_{i}}$. An isolated increase in $m_{i}$ linearly increases the profit 
for a given demand.

If $\omega$ is different from zero (i.e firm $i$ steals retail market share from rival gentailers), we have additional first order effects that originate from firm $i$ 's decision to increase his market share. Despite the fact that a decrease in the market shares of rival gentailers do not affect firm $i$ 's profits directly, the indirect effects of $\Delta \bar{m}_{j}$ are not canceled out. A decrease in $\overline{m_{j}}$ increases generator $i$ 's supply, which in turn increases his marginal costs but also increases his wholesale margin (spot price mark-up) and unambiguously increases $\pi_{i}$. The demonstration of these results and the derivation of the second order effects are in appendix $C$.

Assume that demand follows a geometric Brownian motion, where $\mu$ is the drift, $\sigma$ is the demand volatility and $d W_{t}$ is an increment of a Wiener process.

$$
d D_{t}=D_{t} \mu d t+D_{t} \sigma d W_{t}
$$

This is a standard assumption in the real options literature that generally results in closed-form solutions.

Lemma 5.2.2. Define $\sum_{k=1}^{K} m_{k}$ as $M$. Consider that $\sum_{k=1}^{K} Q C_{k t}$ is a constant $Q C$ and that the marginal cost is equal to $M C_{i}=a+b S_{i}$. Define $h$ as the horizon of the investment. Assume that $r>2 \mu+\sigma^{2}$. The present value $V_{t}$ at $t$ of the project's cash inflows is equal to:

$$
V\left(D_{t}\right)=\tilde{A} D_{t}-\tilde{B} D_{t}^{2}-I
$$

where:

$$
\begin{aligned}
& \tilde{A}=\hat{A} \frac{\left(1-e^{-(r-\mu) h}\right)}{r-\mu} \\
& \tilde{B}=\hat{B} \frac{\left(1-e^{-\left(r-2 \mu-\sigma^{2}\right) h}\right)}{r-2 \mu-\sigma^{2}}
\end{aligned}
$$


and $\tilde{A}>0$ and $\tilde{B}>0$. Proof: appendix C.

The value of undertaking the project is a quadratic concave function of demand $D$. This value depends on initial market parameters $\left(K, M, m_{i}\right.$, $Q C, Q C_{i}, a$ and $\left.b\right)$, on the characteristics of the project $\left(\Delta m_{i}, \omega, I\right.$ and $\left.h\right)$, the risk-free rate $r$ and on the stochastic process for demand ( $\mu$ and $\sigma){ }^{6}$

Define $F(D)$ as the value of generator/gentailer $i$ 's investment opportunity to increase his participation in the retail market for a given realization of $D$. Expanding $d F$ through Ito's Lemma and using standard contingent claims analysis, we have the following quadratic differential equation: ${ }^{7}$

$$
\frac{1}{2} \sigma^{2} D^{2} F^{\prime \prime}(D)+\mu D F^{\prime}(D)-r F=0
$$

Finding the value of generator/gentailer is investment opportunity is not trivial because $V\left(D_{t}\right)$, as defined by equation (5.3), is a quadratic function of $D_{t}$. This means that there is a point where the demand is so high that the value of the project is increased by reduced demand.

Therefore, we have a demand interval where the project is undertaken. Define the the critical lower and upper bounds that trigger the investment as $D_{1}^{*}$ and $D_{2}^{*}$, respectively. The solution of the differential equation above can be attained by separating the problem into three different regions. The first one, given by $\left[0, D_{1}^{*}\right]$, concerns the low demand region where waiting for an increase in demand has equal or greater value than undertaking the project. The second is given by $\left[D_{1}^{*}, D_{2}^{*}\right]$ and reflects the exercise region where the project is undertaken and $F(D)=V(D)$. Lastly, in the region where the demand is greater than $D_{2}^{*}\left(D_{2}^{*}, \infty\right)$ the firm's optimal strategy is to wait for the demand to decrease before initiating the project.

The solution also requires the determination of suitable boundary con-

\footnotetext{
${ }^{6}$ Since $E_{t}^{T}\left(D_{t}^{2}\right)=e^{\left(2 \mu+\sigma^{2}\right)(T-t)} D_{t}^{2}$, the condition $r>2 \mu+\sigma^{2}$ guarantees that the discounted value of the expected demand squared converges.

${ }^{7}$ See Dixit, Pindyck, and Davis (1994) pages 114-117 and 150-151.
} 
Figure 5.1: Concavity of $V\left(D_{t}\right)$

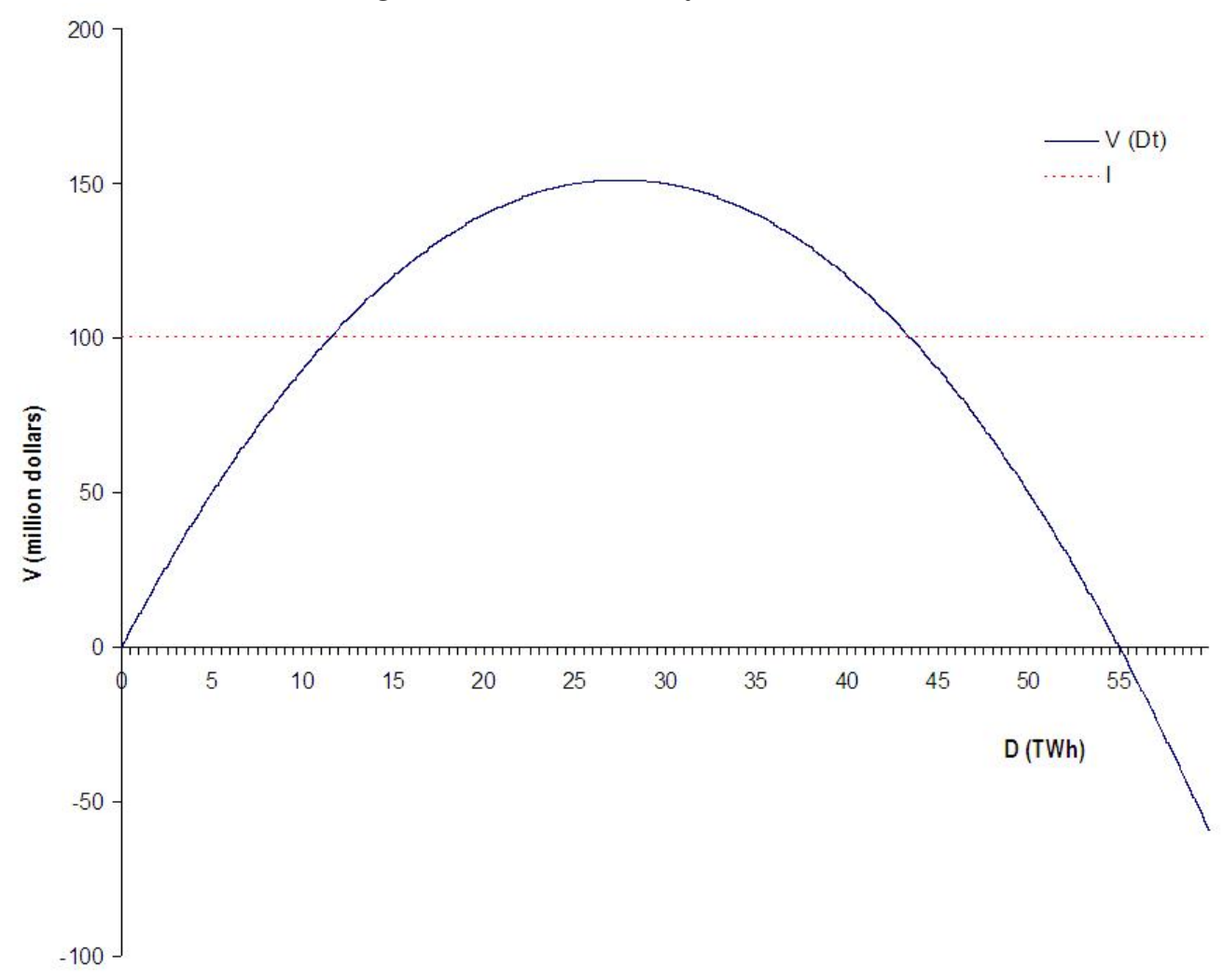

ditions for the first and third regions. If $D \in\left[0, D_{1}^{*}\right]$,

$$
\begin{aligned}
F(0) & =0 \\
F\left(D_{1}^{*}\right) & =\tilde{A} D_{1}^{*}-\tilde{B} D_{1}^{* 2}-I \\
F^{\prime}\left(D_{1}^{*}\right) & =\tilde{A}-2 \tilde{B} D_{1}^{*}
\end{aligned}
$$

If demand, $D$, goes to zero then the value of undertaking the project will remain at $-I .{ }^{8}$ Therefore, as stated by equation (5.5), it is reasonable to assume that the value of the investment opportunity is zero in such a case

\footnotetext{
${ }^{8}$ Notice that, in this case, $F\left(0 \leq D \leq D_{1}^{*}\right)=0$ because the investment will not be taken.
} 
$(F(0)=0)$. The other two conditions come from optimality considerations. Equation (5.6) establishes that the gentailer $i$ receives a net payoff of $\tilde{A} D_{1}^{*}-\tilde{B} D_{1}^{* 2}-I$ upon investment, which is equal to the value of the project if it is undertaken $\left(F\left(D_{1}^{*}\right)\right)$. This is sometimes called the value matching condition. As is usual in the real options literature, equation (5.7) assumes that $F(D)$ is continuous and smooth at the critical exercise demand $D_{1}^{*}$, i.e. $\mathrm{F}\left(D_{1}^{*}\right)$ and $\mathrm{V}\left(D_{1}^{*}\right)$ have the same derivatives. This is also known as the smooth pasting condition. ${ }^{9}$

Conversely, If $D \in\left[D_{2}^{*}, \infty\right)$,

$$
\begin{aligned}
F\left(D_{2}^{*}\right) & =\tilde{A} D_{2}^{*}-\tilde{B} D_{2}^{* 2}-I \\
F^{\prime}\left(D_{2}^{*}\right) & =\tilde{A}-2 \tilde{B} D_{2}^{*} \\
F(\infty) & =0
\end{aligned}
$$

In this case, the value function is decreasing in D. For sufficiently high levels of demand the project is not executed unless demand decreases to the trigger point $D_{2}^{*}$. Again we assume that $F(D)$ is continuous and smooth at this critical point (equations (5.8-5.9)). The greater is the demand from $D_{2}^{*}$ onwards, the smaller is the value of the project opportunity. As stated by equation (5.10), in the limit this value goes to zero. This is often referred to as the transversality condition.

Proposition 5.2.3. The particular solution of the PDE given by equation (5.4) and bounded by equations (5.5-5.10) is the function $F: \Re^{+} \rightarrow \Re^{+}$below:

$$
F(D)= \begin{cases}\alpha_{11} D^{\beta_{1}} & \text { if } D \in\left[0, D_{1}^{*}\right] \\ \tilde{A} D-\tilde{B} D^{2}-I & \text { if } D \in\left[D_{1}^{*}, D_{2}^{*}\right] \\ \alpha_{22} D^{\beta_{2}} & \text { if }\left[D_{2}^{*}, \infty[\right.\end{cases}
$$

\footnotetext{
${ }^{9}$ See Dixit et al. (1994).
} 


$$
\begin{aligned}
& \beta_{1}=\frac{1}{2}-\frac{(r-\mu)}{\sigma^{2}}+\sqrt{\left(\frac{(r-\mu)}{\sigma^{2}}-\frac{1}{2}\right)^{2}+\frac{2 r}{\sigma^{2}}} \\
& \beta_{2}=\frac{1}{2}-\frac{(r-\mu)}{\sigma^{2}}-\sqrt{\left(\frac{(r-\mu)}{\sigma^{2}}-\frac{1}{2}\right)^{2}+\frac{2 r}{\sigma^{2}}} \\
& D_{1}^{*}=\frac{\left(1-\beta_{1}\right) \tilde{A}+\sqrt{\left(1-\beta_{1}\right)^{2} \tilde{A}^{2}+4\left(2-\beta_{1}\right) \beta_{1} \tilde{B} I}}{2\left(2-\beta_{1}\right) \tilde{B}} \\
& D_{2}^{*}=\frac{\left(1-\beta_{2}\right) \tilde{A}+\sqrt{\left(1-\beta_{2}\right)^{2} \tilde{A}^{2}+4\left(2-\beta_{2}\right) \beta_{2} \tilde{B} I}}{2\left(2-\beta_{2}\right) \tilde{B}} \\
& \alpha_{11}=\frac{\tilde{A}}{\beta_{1}} D_{1}^{* 1-\beta_{1}}-2 \frac{\tilde{B}}{\beta_{1}} D_{1}^{* 2-\beta_{1}} \\
& \alpha_{22}=\frac{\tilde{A}}{\beta_{2}} D_{2}^{* 1-\beta_{2}}-2 \frac{\tilde{B}}{\beta_{2}} D_{2}^{* 2-\beta_{2}}
\end{aligned}
$$

Proof: appendix C.

\subsection{Numerical Example}

We now consider a numerical example, approximately based on actual NZEM numbers. We use this example to illustrate possible consequences and results of our theoretical model. Empirical tests and estimations of our VI model are not the scope of this thesis and are left for future research. Our main objective is to set out the potential of the hybrid pricing approach to shed light on the determinants of vertical integration decisions.

Our base scenario corresponds to a vertically integrated market composed of initially symmetric gentailers with $50 \%$ of the retail market ( $K=$ $5, M=0.5$ and $\left.m_{i}=M / K \forall i=1,2, \ldots, K\right)$. Assume the same parameter as used in our illustration $\left(K=5, I=100\right.$ million, $\Delta m_{i}=2 \%, h=4$ and $\omega=60 \%$ ). Consider that the annual aggregate demand at $t$ is equal to 37.5 TWh (the approximate annualized average of the NZEM offtake between $22 / 01 / 2004$ and $30 / 11 / 2010$ ). Suppose that 10 TWh is hedged through 
forward contracts $(Q C=10)$ and remains fixed during the project's horizon. The base scenario considers average levels of vertical integration and hedge coverage. We analyze the project value under different scenarios. In equilibrium, the average annual supply is equal to $7.5 \mathrm{TWh}(\bar{S}=D / K)$. Consider a spot price of approximately NZD72.50/MWh (Haywards node average between $22 / 01 / 2004$ and $30 / 11 / 2010$ ). We know by equation (3.5) that marginal costs are equal to spot prices $(p=M C)$ in fully vertically integrated economies (see chapter 3 ). In this example, we assume that $a=50$ and $b=3$ to keep the same average marginal cost $(p=M C=$ $a+b * 7.5=72.5)$ as in the empirical exercise of chapter $4 .{ }^{10}$ In addition, assume the risk free rate is $r=0.06$, the annual demand growth rate to be $\mu=0.0135$ (the NZEM annual average since 1997), the volatility to be $\sigma=0.08$ and the retail price to be fixed and equal to $p^{R}=N Z D 150.00$.

As shown by Figure 5.2, the risk-free rate has the standard impact on the value of the investment opportunity. An increase in $r$ raises the opportunity cost of the project and diminishes the value of the investment opportunity $F$. However, the impact on the timing of the project is more ambiguous. The triggers $\left(D_{1}^{*}\right.$ and $\left.D_{2}^{*}\right)$ in each scenario are represented by the squares. An inspection of equations (5.13-5.14) and Figure 5.3 shows that the impact on the timing of the investment differs. This happens because the impact of $r$ on $D_{1}^{*}$ and $D_{2}^{*}$ is not monotonic ( $r$ affects not only the characteristic roots but also the parameters $\tilde{A}$ and $\tilde{B}$ ). In this example, an increase in $r$ slightly postpones investments in both high $\left(D>D_{2}^{*}\right)$ and low $\left(D<D_{1}^{*}\right)$ demand scenarios.

Figure 5.3 shows that changes in the demand growth rate $\mu$ have an ambiguous effect on both the value of the investment opportunity and the timing of the investment. An increase in $\mu$, increases the expected demand which in turns raises the expected amount of electricity sold. On the other hand, since the retail price $p^{R}$ is fixed, an increase in $\mu$, increases the expected spot price and decreases the expected margin. The overall

\footnotetext{
${ }^{10}$ Recall that $\rho=0$ in this exercise since demand is the only state variable.
} 
Figure 5.2: Project value and investment policy (different risk free rates)

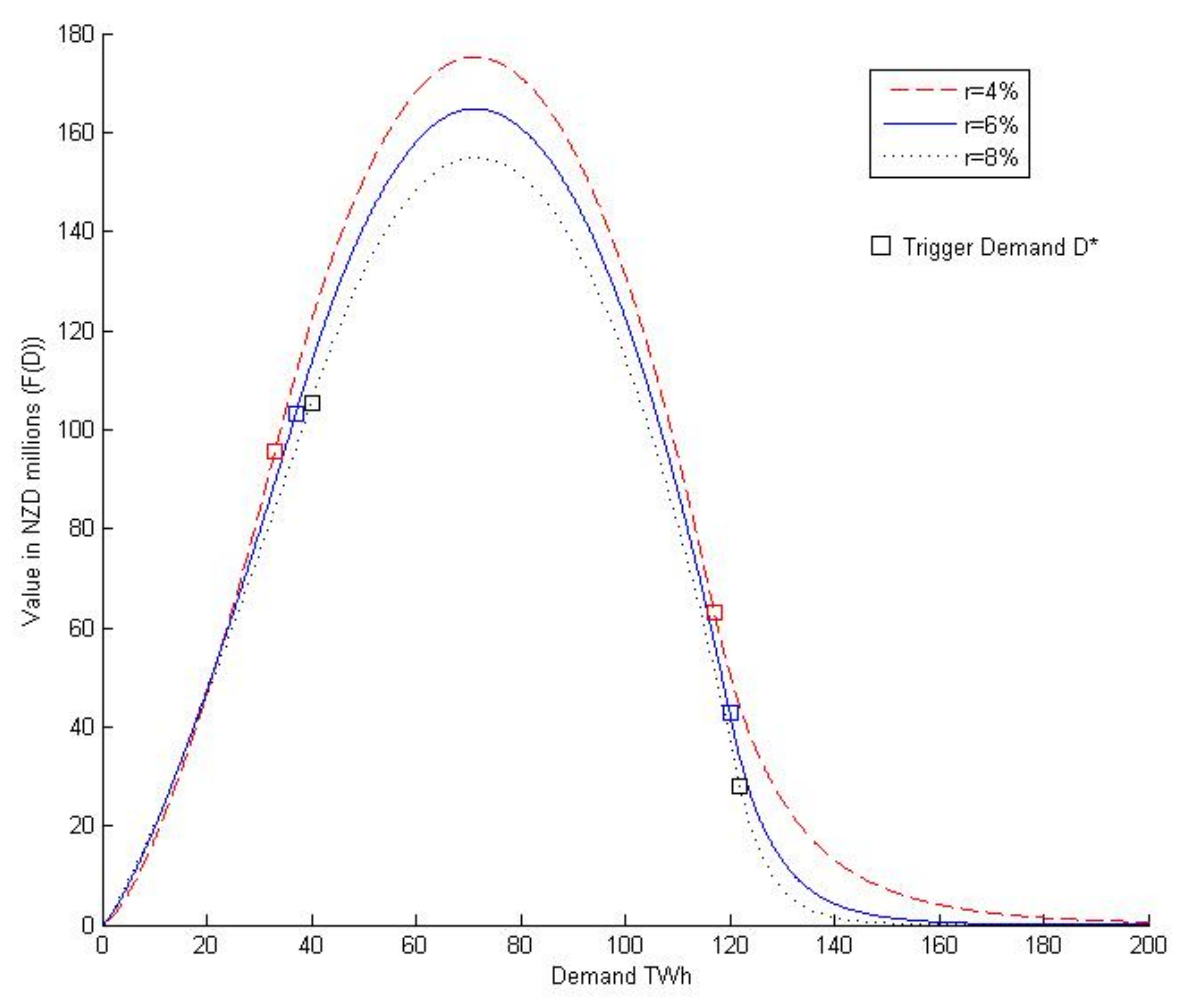

net effect on the value of undertaking the project (payoff) depends on the market parameters. In our example, decreases in $\mu$ postpone the investments for low values of demand and anticipate it for high values of demand. The effect on the value of the investment depends on the level of demand. For example, for sufficiently extreme values of the demand (low or high), the highest value of the project opportunity occurs when $\mu=0$. The opposite happens for intermediate values of demand. This happens because the quadratic term of the payoff function $V(D)$ decreases since $B \geq 0$. For longer investment horizons, it is reasonable to expect that drifts greater than zero $\mu>0$ would significantly affect the retail price. 
Figure 5.3: Project value and investment policy (different drifts)

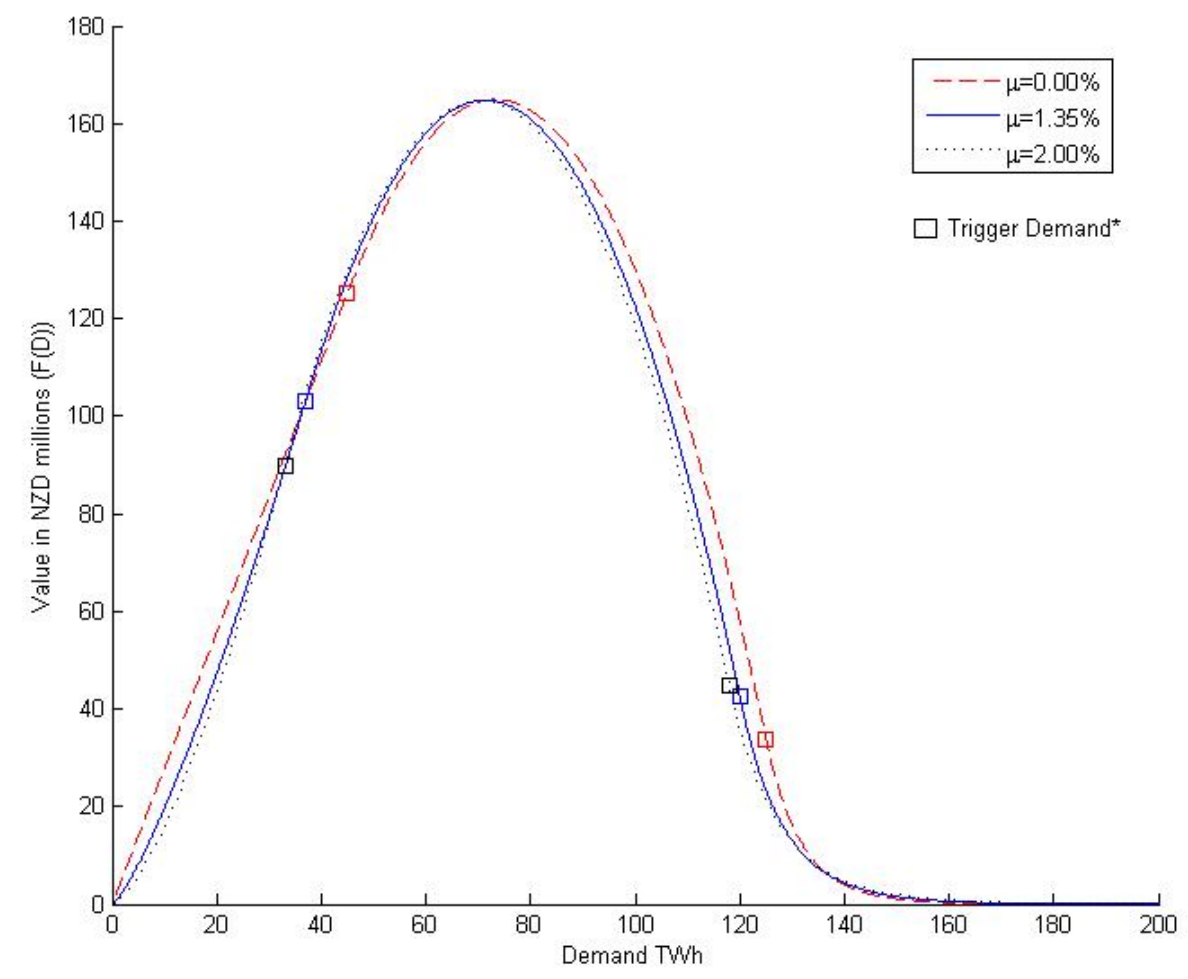

Figure 5.4 shows that volatility has negative impact on the value of the investment in the exercise range. An increase in $\sigma$ decreases the value of the option to wait when the demand is smaller than $D_{1}^{*}$ and also decreases the value of the undertaken project in the exercise region $\left(D_{1}^{*}<D<D_{2}^{*}\right)$. However, the effect of volatility on $F(D)$ is positive for a sufficiently high demand level. These results reflect the functional form of the payoff function $\left(\max \left(V\left(D_{t}\right)-I, 0\right)\right)$. Unlike standard single variable American call options whose payoffs are convex in terms of their underlying state variable, here, the payoff of undertaking the project presents both convex and concave regions with regards to demand $D$. Inspection of the dash-dot line in Figure 5.5 shows that the payoff function is typically convex in 
Figure 5.4: Project value and investment policy (different volatilities)

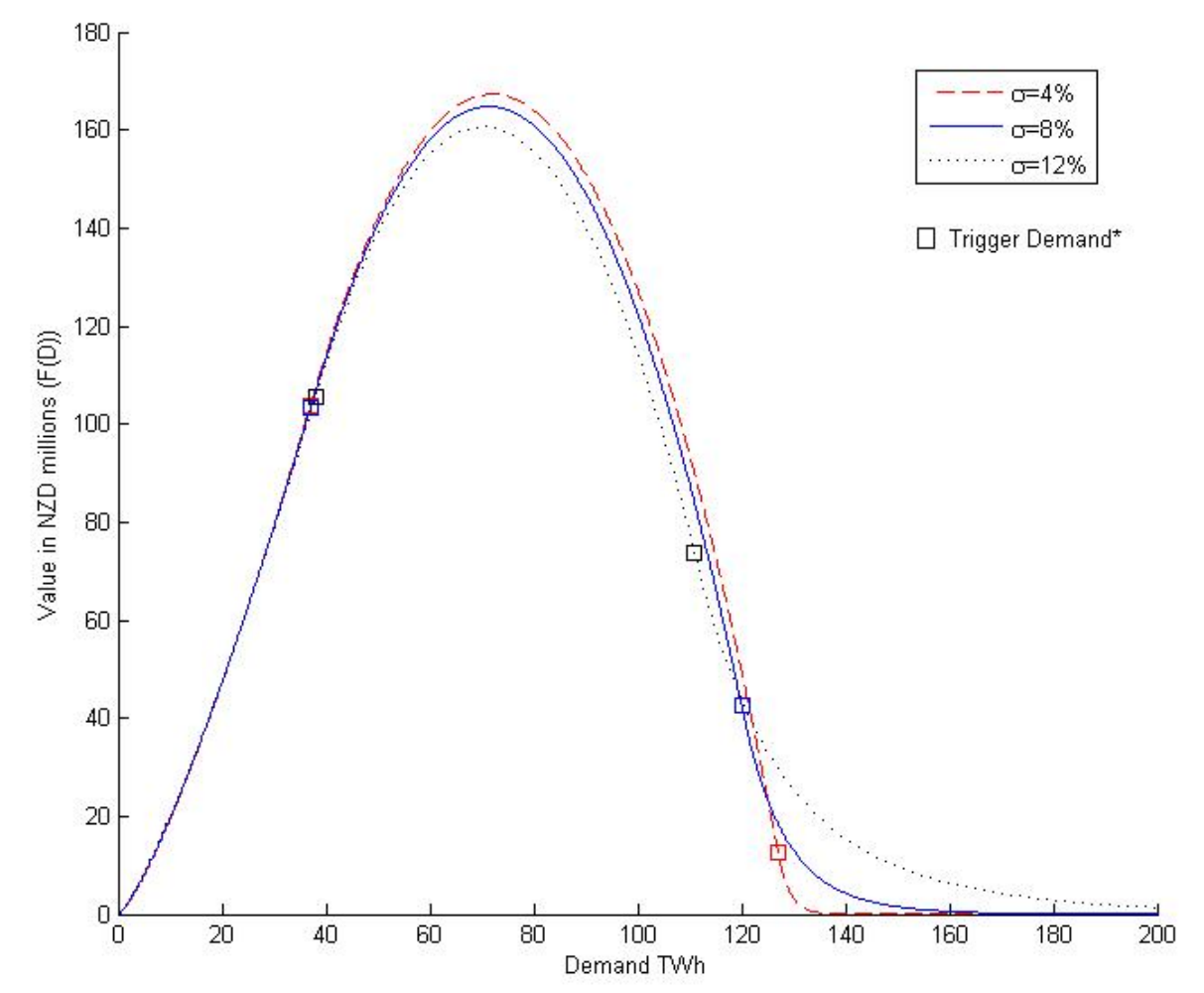

the non-exercise regions $\left(D<D_{1}^{*}\right.$ and $D>D_{2}^{*}$ ) and concave in the exercise region. This means that the effect of volatility on the call option $F$ depends in which region the average demand lies. Clearly, the concave region dominates in the given example. We observe from Figure 5.6 that an increase in the number of firms increases the value of the opportunity to invest in retail expansion. The main reason is that the more competitive is the market, the greater is the retail margin for a given retail price. In the considered example, $D_{1}^{*}$ that triggers the decision to undertake the investment is greater in markets with smaller numbers of generators (say, $K=4$ versus $K=6$ ). The same happens to $D_{2}^{*}$. This means that, in this example, 
Figure 5.5: Concavities of $F(D)$ and V(D)

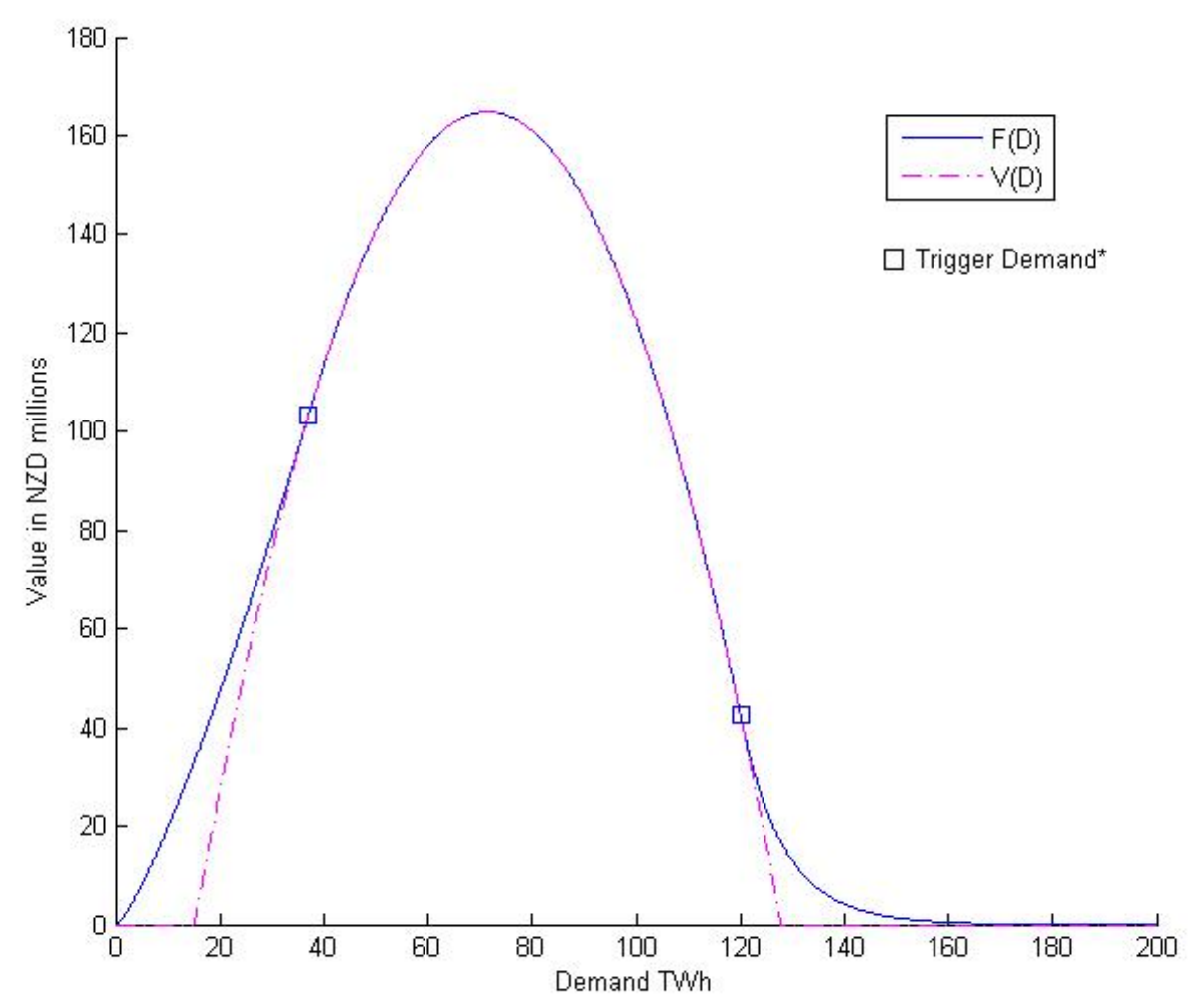

the investments will be undertaken in competitive markets later than in non-competitive markets when the demand is low $\left(D<D_{1}^{*}\right)$ but are less likely to be prevented by high demand outcomes.

Figures 5.7 and 5.8 respectively show that the effect of the generators' forward contracts $(Q C)$ and the degree of vertical integration $(M)$ over both the value and timing of the investment in retail expansion follow a similar pattern. Given $p^{R}$ and $Q C_{i}$ (firm $i$ 's hedging position), Markets with greater $Q C$ have ceteris paribus a smaller electricity spot price and a bigger retail margin (see equation (3.7)). The same happens for $M$ for a given firm $i$ 's market share $\left(m_{i}\right)$. In particular, ceteris paribus, Figure 
Figure 5.6: Project value and investment policy (different number of firms)

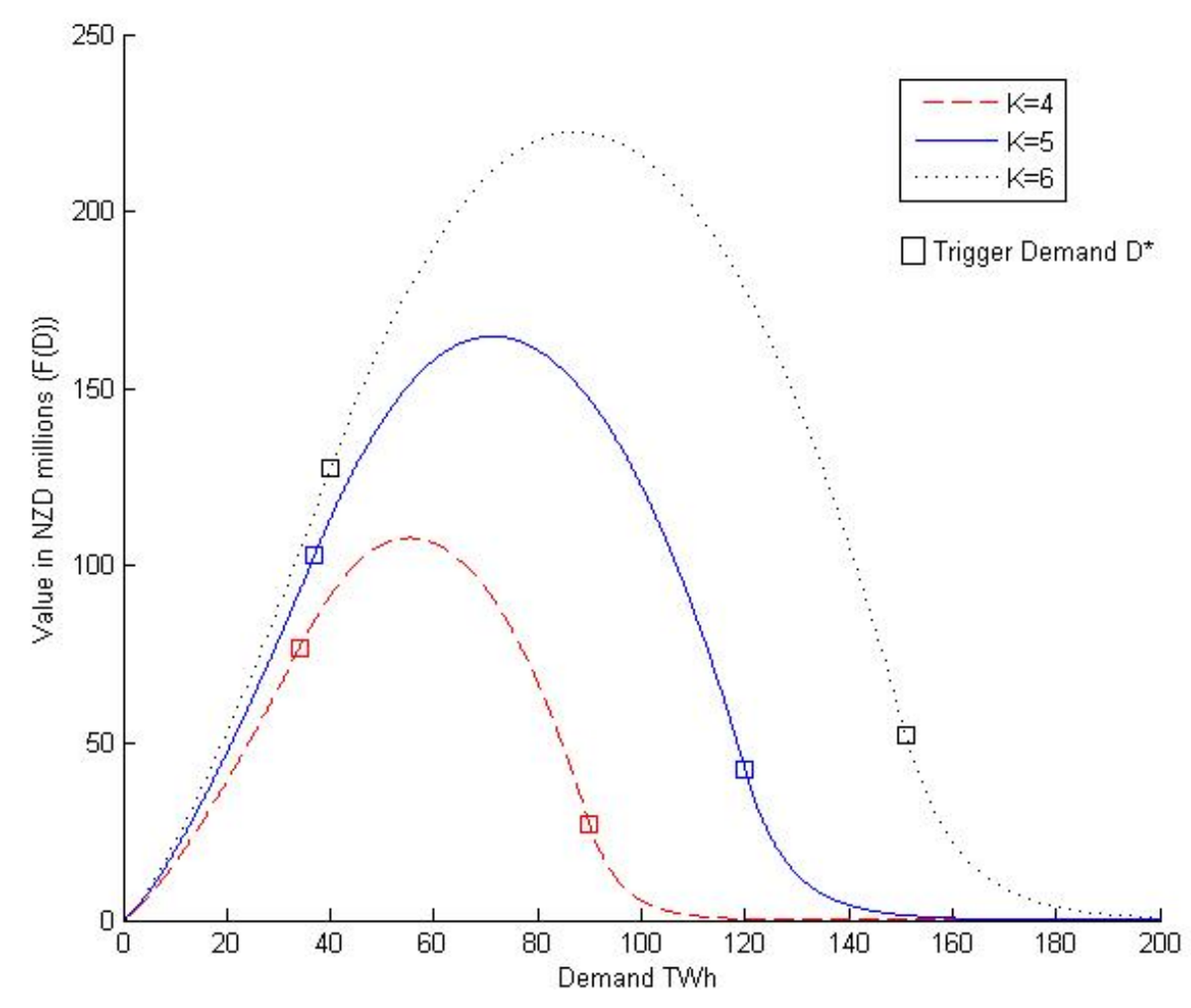

5.7 shows that a decrease of forward contracts $Q C$ would decrease the project value but would not affect significantly the decision to undertake the project. In the most extreme (and unrealistic) case, where the retail expansion of $2 \%$ decreases the gentailers' financial hedges to zero $(Q C=0)$, the changes on both timing and value of the project are very small.

Figure 5.9 shows that changes in the retail price have the expected result on both the value and timing of increasing vertical integration. Projects with smaller retail prices are postponed and have smaller values. Since the retail price is fixed in our framework, a decrease in $p^{R}$ directly decreases the profitability of the investment (with no short-term effect on demand). 
Figure 5.7: Project value and investment policy (quantity contracted)

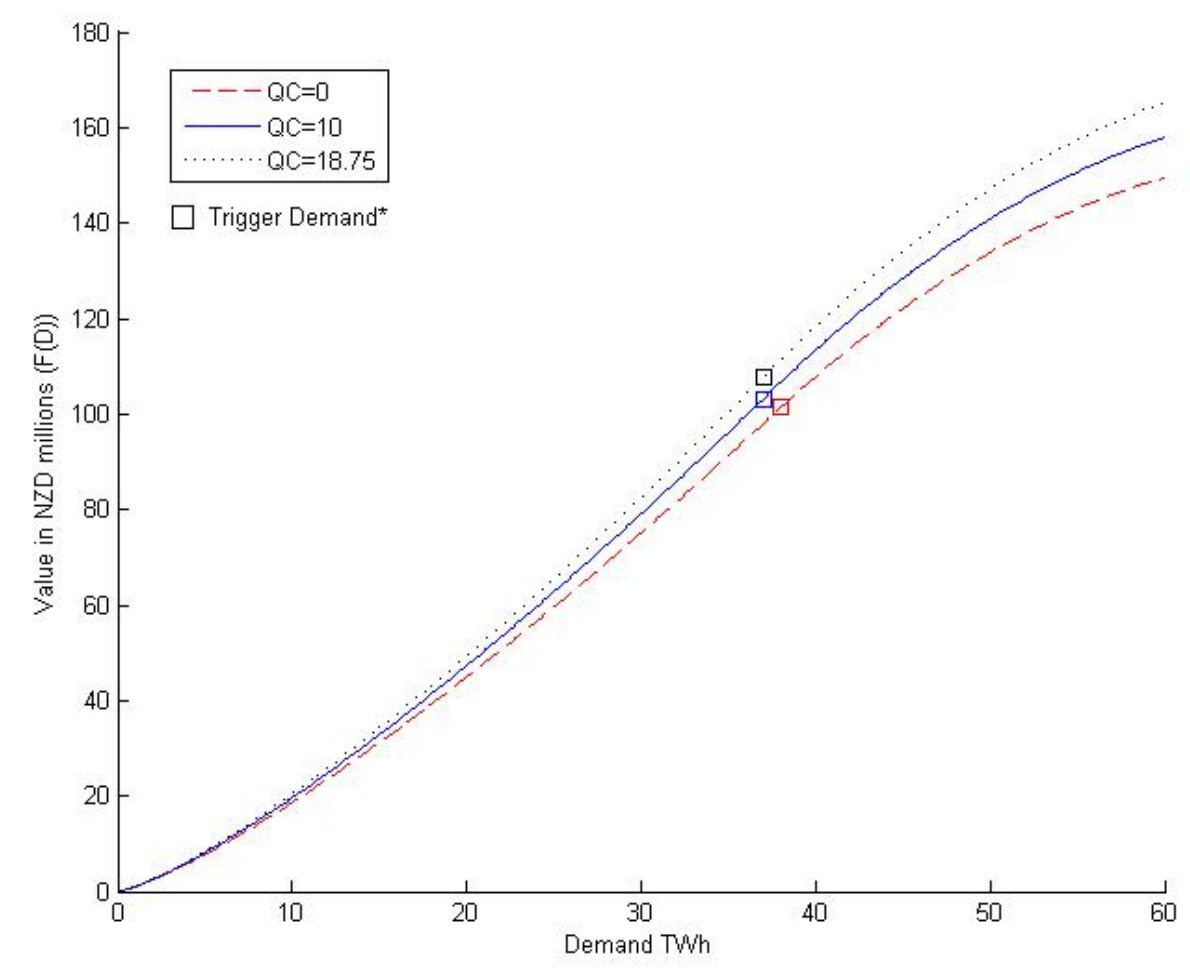

In this example, the demand interval which triggers the gentailer $i$ 's retail market expansion is considerably shorter for $p^{R}=120$ than for $p^{R}=170$.

In summary, in electricity markets where retail prices are relatively fixed, the gentailers' decision to undertake sunk investments (e.g marketing campaigns) to increase their participation in the retail market is affected both by market equilibrium parameters and the level of demand ( $D<D_{1}^{*}$ or $D>D_{2}^{*}$ ). In particular, because of the rigidity of $p^{R}$, the project's payoff is potentially concave and can imply an unusual negative relationship between volatility and the option to wait. In addition, aspects such as market power and the degree of vertical integration play an important role in the retail expansion decision. While the example, is somewhat 
Figure 5.8: Project value and investment policy (degree of vertical integration)

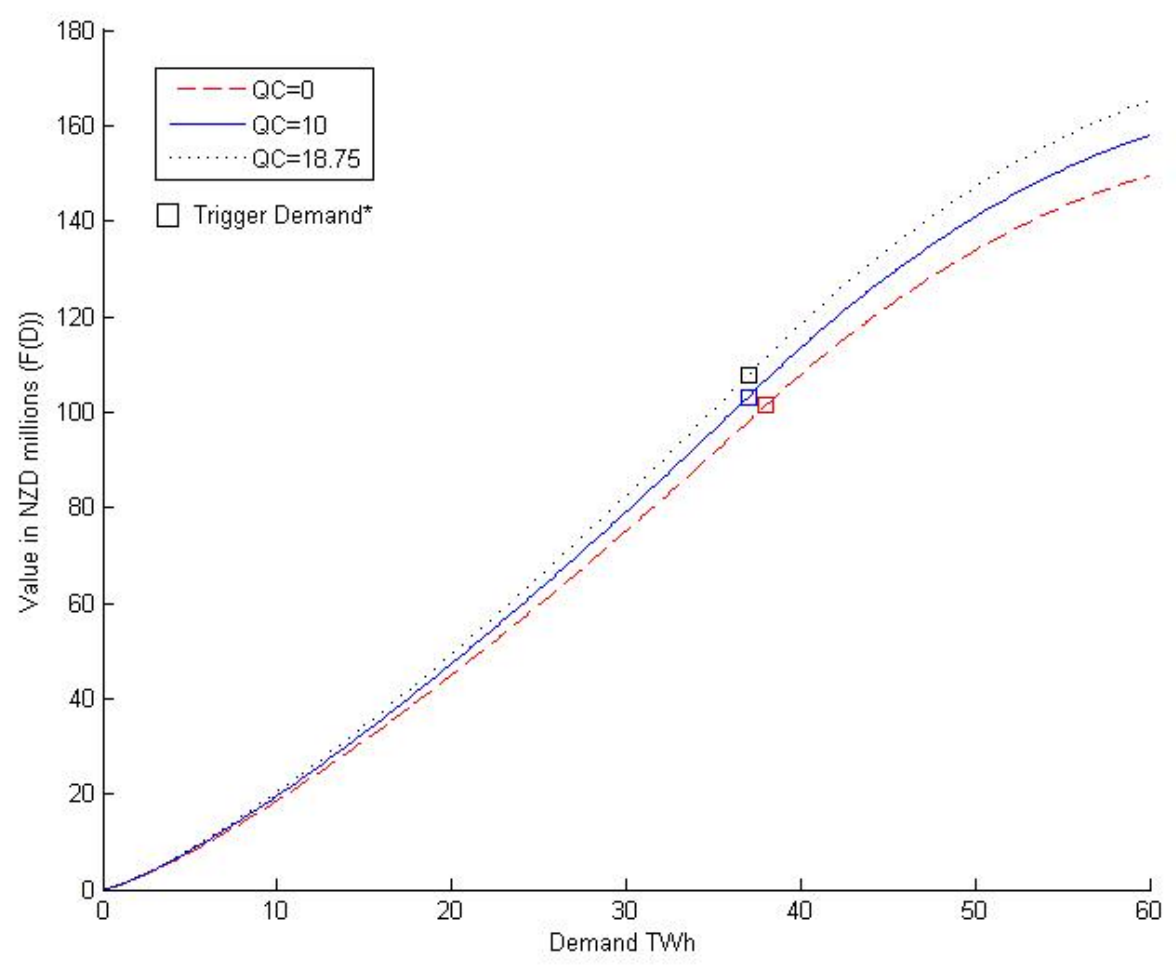

stylised, it does suggest that market frictions arisen from unrecoverable costs can be an important factor in market/region entry decision for retailers. 
Figure 5.9: Project value and investment policy (Retail prices)

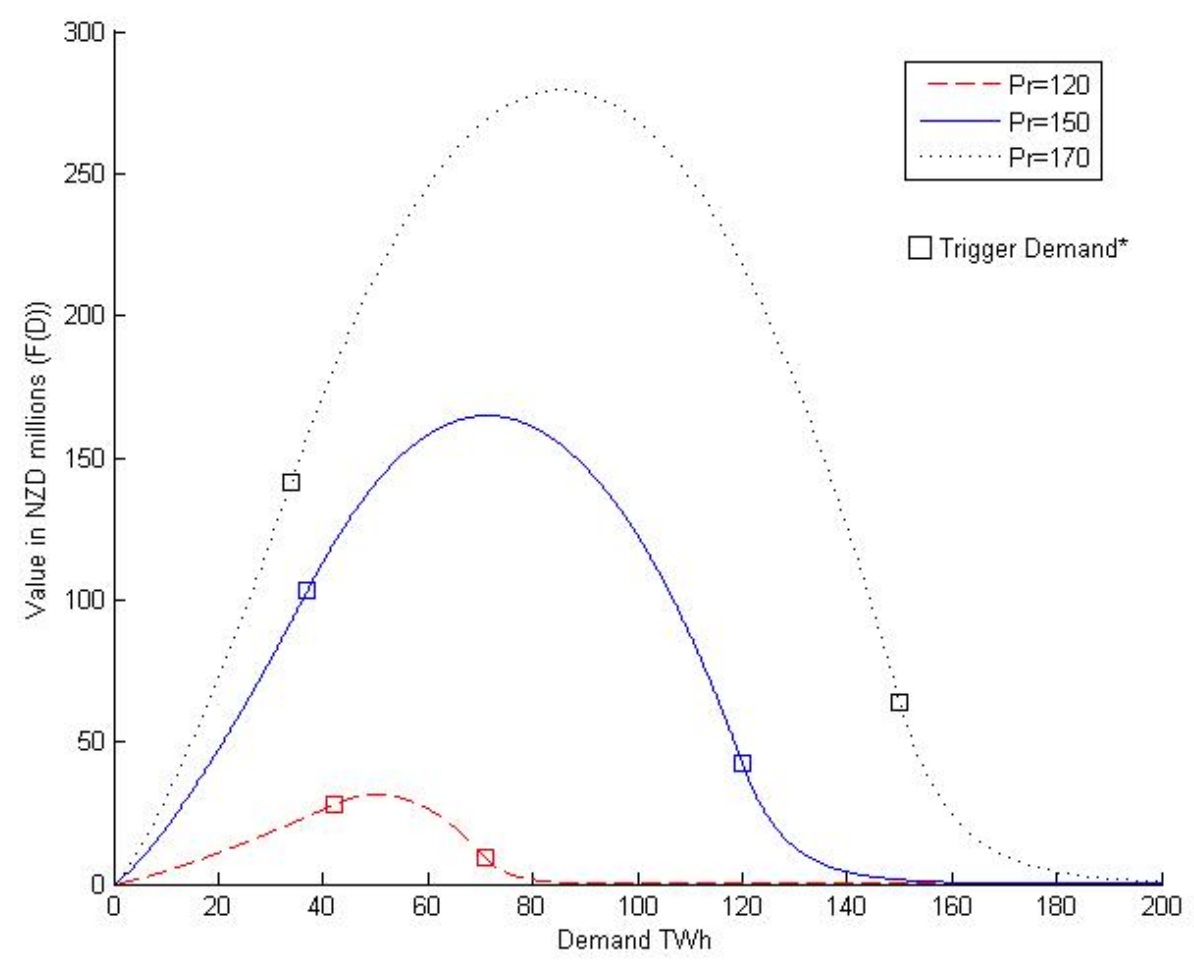




\section{Chapter 6}

\section{Market power and hedging decision}

The interaction between exogenous forward contracts and spot market power in electricity wholesale markets has been the subject of a considerable literature. However, the relationship between risk, spot market power and hedge pricing have not yet received proper attention. We propose to address this relationship where the spot market obeys a uniform-price auction clearing mechanism and where the hedging decision faces uncertainty on both demand and supply. In our approach we have equilibria in the spot and hedge markets. These aspects are common to many competitive wholesale electricity markets.

The relationship between forward markets and market power was first presented by Allaz and Villa (1993). The authors developed a duopoly model with Cournot competition, linear demand, and constant marginal costs, and concluded that an increase in exogenously set forward commitments pushes the spot market towards a competitive solution. Papers such as Newbery (1998), Green (1999) and Bushnell (2007) followed the same cost, demand and forward price assumptions and extended Allaz and Villa (1993) to deal with more generic oligopolistic frameworks, greater number of firms, capacity constraints and market entry. These pa- 
pers also find that exogenous forward markets reduce wholesale market power. ${ }^{1}$ This result is confirmed by empirical papers such as Wolak (2000) and Bushnell et al. (2008). A common feature of all these models is the assumption that production decisions are made at a particular point in time and all the hedging decisions affecting this particular point in time are taken in previous periods in some unspecified way. They share the limitation of considering an (almost) perfect foresight of demand and supply in the exogenously setting of the forward price. ${ }^{2}$ In other words, (expected) spot prices are equal to forward prices and the risk and possible market power determinants of hedging decisions are neglected.

Another group of papers use an equilibrium framework to relate risk and economic fundamentals to contract decisions in electricity markets. However, most of them rely on the perfect competition assumption. An important representative of this equilibrium literature on optimal hedging is Bessembinder and Lemmon (2002). The authors use a mean-variance approach and analyze the impact of demand uncertainty, in a context of non-linear costs, on the forward premium and hedging quantities under perfect competition. They find that forward contracts are influenced by the risk premium, price volatility and price skewness. Porchet, Ad, Touzi, and Chemla (2009) extend the model to account for vertical integration but still rely on the perfect competition assumption. Anderson and $\mathrm{Hu}$ (2008) offer an original approach, in which they combine a duopoly structure in

\footnotetext{
${ }^{1}$ On the other hand, Ferreira (2003), Mahenc and Salanie (2004), Liski and Montero (2006) and Green and Le Coq (2006) find opposite results using Bertrand models or focusing on the dynamic aspects of contracts.

${ }^{2}$ Neither of these papers consider $Q C$ or $P C$ as endogenous variables. Besides, uncertainty is either neglected (Allaz and Villa (1993) and Bushnell (2007)) or modeled as a very minor aspect. For example, Newbery (1998) and Green (1999) discuss the potential implications of uncertainty for their results. They respectively conclude that shocks in price and demand do not change their main findings. However, they both assume risk neutrality of all agents in the market and adopt very particular and predictable probability distributions for shocks in demand (exclusively). That is, Newbery (1998) assume that generators take account of reasonably predictable variations in demand over the day and Green (1999) argues that his results would be the same considering a normally distributed demand intercept with small volatility.
} 
the generation market with uncertainty. The authors consider the retailers' perspective and show that market power affects forward decisions and can by itself justify the existence of a forward premium. However, they assume risk neutrality of both generators and retailers and no capital constraint, neglecting a major purpose of hedging, i.e. risk aversion (see section 6.1 for explanation).

An important drawback of all these papers is that they consider the key specific elements in isolation, and they do not consider uncertainty in costs. In reality, no matter the time horizon, generators' marginal costs are possibly influenced by random and volatile variables (e.g. input prices, shadow prices relating to storage level of water or other fuel and operational constraints to capacity). ${ }^{3}$ Uncertainty in costs can have different implications for hedging decisions than the commonly assumed demand randomness.

Our model takes into account costs and demand uncertainty in an electricity market characterized by risk aversion, oligopoly, vertical integration and a uniform-price auction clearing mechanism. We develop a twoperiod framework under the usual assumptions of linearity of demand and marginal costs. We analyze the implications of these assumptions for the forward premium and optimal hedging quantum. Moreover, we show that uncertainty and volatility have implications for the exertion of market power. The spot price mark-up, measured by the Lerner index, is not only affected by strategic conduct (in our model represented by Cournot behavior and the number of firms), but also by exogenous stochastic variables that influence demand and costs. In other words, in highly concentrated electricity markets with forward contracts, the usual measure of market power can reflect risk management instead of solely oligopolistic conduct.

This chapter is organized as follows. In section 6.1, we discuss the role of preferences on hedging decision. Sections 6.2 and 6.3 analyze the case of a fully separated electricity market, where generators and retailers are all

\footnotetext{
${ }^{3}$ See Evans and Guthrie (2009).
} 
different firms. We use numerical exercises in section 6.4 to infer the possible implications of risk and market concentration on forward premium, on the hedging quantity and on the expected spot price mark-up. Section 6.5 looks at the theoretical and numerical changes on the same three measures (forward premium, hedging quantities and expected Lerner index) under the presence of vertical integration (VI).

\subsection{The role of preferences}

The general literature on determinants of corporate hedging has two parallel approaches. The first one is founded on papers such as Stulz (1984) and focuses on how hedging policy responds to corporate frictions related to a) tax rules; b) transaction costs of bankruptcy and c) agency costs. Papers such as Stulz (1990), Bessembinder (1991) and Froot, Scharfstein, and Stein (1993) argue that firms can benefit from hedging market risks because excessive volatility increases the expected costs of financial distress and can lead to suboptimal investment.

The second approach relies on arbitrage pricing methodologies and the timing aspects of hedging. This literature starts from the premise that corporate hedging does not add value to the firm in complete markets with no capital frictions. According to this approach, no-arbitrage assumption holds and preferences do not matter. Frictions and constraints such as illiquidity arise as possible determinants of hedging. The firm's optimal hedging strategy is the one that maximizes its value given such constraints. Mello and Parsons (2000) and Boyle and Guthrie (2006) are representative of this line of research. Mello and Parsons (2000) argue that popular hedging strategies such as minimizing the variance of the firm's cash flow or revenue are suboptimal because they ignore the relationship between cash flow in the next period and the marginal value of cash balance. Boyle and Guthrie (2006) show that hedging can have the effect of reducing investments. Their model shows that hedging could add value 
by allowing investments to be delayed in situations where capital frictions would cause it to commence prematurely.

According to Cartea and Geman (2009), however, the non storability of electricity renders useless the usual arguments of cash and carry, dynamic hedging and buy-and-hold strategies which are the basis of no-arbitrage arguments employed in the commodity derivatives literature. Liquidity and other corporate frictions are not the only constraints to firms' optimal decisions. The hedging policy itself is further limited when applied to the electricity market in incomplete markets. As observed by Willems and Morbee (2008), the extent to which a firm can hedge its exposure depends on the availability of markets, their liquidity and the presence of speculators who can absorb part of the risk. According to the authors, these factors change as markets evolve from pure over-the-counter (OTC) markets to more complete markets in which there is a liquid trade in a broad set of derivatives. Unfortunately, electricity contracts are mostly traded in OTC markets and a "liquid trade of a broad set of derivatives" is a rare exception in this industry. As discussed in chapter 1, preferences and risk aversion are closely connected to asset pricing in markets characterized by incompleteness. Thus, decision modeling in incomplete markets is intrinsically related to utility functions (preferences).

As argued by Bessembinder and Lemmon (2002) and Porchet et al. (2009), the mean-variance approach can offer useful insights to the hedging decision analysis despite its known limitations. It is reasonable to assume that managers trade-off between increasing their expected profit and decreasing their risk in proportion to their risk aversion. As mentioned, the idea of a firm penalizing risk is associated in the corporate finance literature with liquidity, transaction and agency costs that arise from an increase in the probability of default. The mean-variance approach also offers a tractable way to model incomplete markets such as electricity. The mean-variance function, under certain conditions, corresponds to a 
second-order approximation to generic utility functions. ${ }^{4}$

The following section will explore an equilibrium approach in the spirit of papers such as Bessembinder and Lemmon (2002), Porchet et al. (2009) and Willems and Morbee (2010). In particular, we use utility functions to model electricity firms' decisions. We also adopt the mean-variance approach to encapsulate market frictions and incompleteness in a tractable framework.

\subsection{Basic assumptions and spot market results}

Consider the following structure. Firms do not participate simultaneously in the retail and generation business (no vertical integration). Each firm's decision is made by a utility maximizing agent. The electricity market has $N$ total firms made of $K$ generators and $R$ retailers. Generators are symmetric and oligopolistic. Retailers' revenues are determined by an exogenous retail price and their respective market share of consumers' demand. Demand and generators' cost functions are influenced by a given set of stochastic state variables. Players know the probability distribution of stochastic variables. As shown in Figure 6.1, our model has three pe-

Figure 6.1: Timing framework

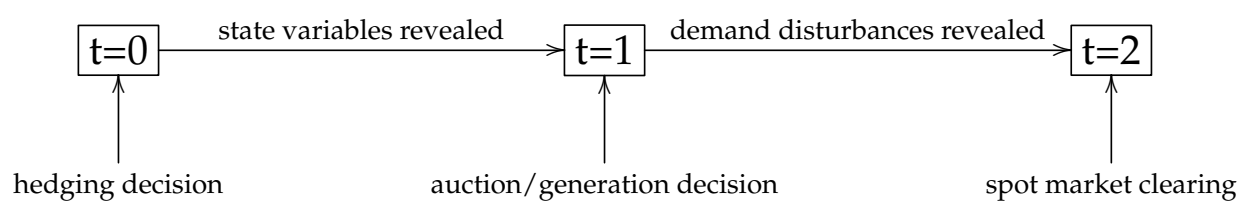

riods. At a point in time in the first period $(t=0)$ the hedging decision is made by generators and retailers. The forward market clears. At the second period $(t=1)$, state variables are revealed and generators decide

${ }^{4}$ See, for example, Baron (1977), Levy and Markowitz (1979) and Pulley (1981). 
their production through a uniform price auction. Despite the knowing the state variables in $t=1$, some uncertainty about the final realization of the demand remains. At the third period $(t=2)$, the demand is completely revealed and the electricity is delivered. The wholesale spot market clears. Notice that the time duration between these dates is unlikely to the same and depends on electricity market rules. ${ }^{5}$ The nature of choice in this model is essentially recursive. Firms choose their optimal supply, $S^{*}(Q C)$, at $t=1$ for a given $Q C$ and choose the optimal quantity contracted, $Q C^{*}$, at $t=0$ taking their optimal supply into account. All the assumptions will be now formalized and motivated.

Assume that definitions (2.1.1-2.1.10) of the basic model described in chapter 2 hold and also that the assumptions of proposition 2.2.3 are valid. That is, at $t=1, W$ is known and the stochastic aggregate demand $\tilde{D}$ is formed by a deterministic component $D=c-\kappa_{o} p^{R}+\sum_{j=1}^{L} \kappa_{j} w_{j}$ and an additive shock $\epsilon$. The deterministic component is affected by the price charged to final consumers $p^{R}$ (retail price) and by demand shifters. The retail price can either be assumed to be a state variable or to be a known constant. Frequently, the literature considers a unique state variable represented by the observed demand itself (say, $D=w_{1}$ ). However, we can also think of demand shifters as including income, activity, institutional changes, seasonality and climatic factors. The stochastic component $\epsilon$ is unknown at $t=0$ and $t=1$ and represents short-term demand fluctuations that occur between the auction and the specific time of delivery at $t=2$. Hence, the demand $\tilde{D}$ is totally revealed only at $t=2$.

As stated in proposition 2.2.3, we assume that generators have a symmetric and well behaved quadratic cost function. The total cost of each generator $i$ in $t=1$ is $C_{i}=\frac{b}{2} S^{2}+\left(a+\sum_{j=1}^{L} \rho_{j} w_{j}\right) S+F$ for all $i=$ $1,2, \ldots, K$. Therefore, the marginal cost $M C_{i}\left(S_{i}, \vec{W}\right)$ of each generator is linear: $M C_{i}=a+b S_{i}+\sum_{j=1}^{L} \rho_{j} w_{j}$.

\footnotetext{
${ }^{5}$ In the NZEM, for example, forward contracts can be traded several months before the energy dispatch and the uniform-price auctions are undertaken in less than a day from delivery.
} 
This assumption addresses the potential impact of generators' marginal cost shifters such as capacity utilization, temperature, water precipitation or shadow prices of inputs (such as gas, water or other fuel). It also takes into account abrupt increases in the marginal cost of generation associated with non-storability of electricity, which are well documented as a reason for the existence of the so-called spikes (see e.g. Deng (2000) and Hughes and Parece (2002)). The assumption of linearity of marginal cost is common in the theoretical literature on the impact of exogenous forward contracts over the spot market power. The assumption of symmetry of generators simplifies our results.

Definition 6.2.1. Retailer i's market share is fixed and defined by $m_{i}$. Therefore, his demand is $m_{i} \tilde{D}\left(p^{R}, \vec{W}\right)$ and, by construction, $\sum_{i=1}^{R} m_{i} \tilde{D}\left(p^{R}, \vec{W}\right)=$ $\tilde{D}\left(p^{R}, \vec{W}\right)$.

Retailers' demand is assumed to be a fixed proportion of $\tilde{D}$ and, by construction, total retailers' demand must be equal to aggregate consumer demand. The exogeneity of the market share $m_{i}$, of all $i=1,2, \ldots, R$, means no significant change in the retailers' participation in the market during the model horizon. It is certainly reasonable from a short-term perspective and it is a simplification that is not far from reality also in the long-term for stable electricity retail markets (e.g. NZEM). In our model, retail market variables $m_{i}$ and $p^{R}$ are taken as given. This chapter focus is on the determinants of the spot and forward market equilibrium.

Definition 6.2.2. There is one forward market only, attended by generators and retailers (no outside speculators). Assume $Q C_{i}^{*}$ the generator i's optimal quantity contracted (chosen at $t=0$ ). The forward market is cleared at $t=0$ and the equilibrium forward price $P C^{*}$ determined (so that $\sum_{i=1}^{N} Q C_{i}^{*}\left(P C^{*}\right)=0$ ).

We simplify the forward transactions by assuming that they can be approximated by a unique forward market cleared by a known equilibrium price $P C$. Issues related to liquidity or the nature of bilateral transactions will not be analyzed here. Bessembinder and Lemmon (2002) argues 
that, while the assumption of absence of speculators in the market is an over-simplification of actual markets, it is a reasonable starting point since speculators rarely can take part in the physical electricity delivery market, which is responsible for the great majority of forward transactions. Following their argument, we also assume that there are no speculators.

Decisions have a recursive nature. Generators simultaneously submit supply schedules $\hat{S}_{i}$ at $t=1$. Considering each firm's bid, the auctioneer computes at $t=2$ the equilibrium price $p^{c}$ that satisfies the market clearing condition. Bidders can infer rivals' optimal contract strategies (adopted in $t=0)$ since there is no asymmetry of information and firms agree about the probability distribution of stochastic variables. Therefore, at the moment of the auction $(t=1)$, from the perspective of firm $i$, uncertainty about price is due exclusively to the uncertainty about the short-term demand fluctuation $\epsilon$ revealed at $t=2$.

Definition 6.2.3. Generator i's ex-post profit upon the realization of the market clearing price at $t=2$ is:

$$
\begin{aligned}
\pi_{i}^{G}= & S_{i}\left(p^{c}, Q C_{i}, \vec{W}\right) p^{c}-C_{i}\left(S_{i}\left(p^{c}, Q C_{i}, \vec{W}\right), \vec{W}\right) \\
& +\left(P C-p^{c}\right) Q C_{i},
\end{aligned}
$$

and his ex-post operating profit at $t=2$ is

$$
\Upsilon_{i}=S_{i}\left(p^{c}, Q C_{i}, \vec{W}\right) p^{c}-C_{i}\left(S_{i}\left(p^{c}, Q C_{i}, \vec{W}\right), \vec{W}\right) .
$$

There are two sources of payoff for generator $i=1 \ldots N$ at time $t=1$. The operating profit from the generation activity, here defined by $\Upsilon_{i}=$ $p^{c} S_{i}-C_{i}$, and the revenue $\left(P C-p^{c}\right) Q C_{i}$ from forward market transactions.

Definition 6.2.4. Retailer $i$ 's ex-post profit upon the realization of market clearing price at $t=2$ is:

$$
\pi_{i}^{R}=m_{i}\left(p^{R}-p^{c}\right) \tilde{D}\left(p^{R}, \vec{W}\right)-\left(p^{c}-P C\right) Q C_{i}
$$


That is, retailer i's purchase is determined by his exogenous retail market participation $m_{i} \tilde{D}\left(P^{R}, W\right)$. It also means that there is no purchase decision for retailers since the spot price is determined by suppliers' strategies and the exogenous aggregate demand. However, retailers are active players in the forward market.

Definition 6.2.5. The owner of firm $i$ has the following maximization problem if he is a generator:

$$
\max _{Q C_{i}, \hat{S}_{i}} E_{W, \epsilon}\left[U_{i}\left(\pi_{i}^{G}\right)\right]=\max _{Q C_{i}} E_{W}\left[\max _{\hat{S}_{i}} E_{\epsilon \mid W}\left[U_{i}\left(\pi_{i}^{G}\right)\right]\right],
$$

and the following maximization problem if he is a retailer:

$$
\max _{Q C_{i}} E_{W, \epsilon}\left[U_{i}\left(\pi_{i}^{R}\right)\right]
$$

where $W, \epsilon$ are random variables and $\hat{S}$ is the supply schedule.

Recall that decisions are taken recursively. Firm $i$ chooses his bid schedule $\hat{S}_{i}(p)$ (if he represents a generator) and decides about hedging $Q C_{i}$ in order to maximize his expected utility. The general uncertainty is given by the joint distribution of the state variables $W$ and the short term demand shock $\epsilon$. However, as illustrated by Figure 6.1, the timing and information are different for both decisions. Thus, the agent problem can be split into two separate decisions and solved recursively. The agent bids the supply schedule at $t=1$ that maximizes the expected utility taking the state variables as given and then he decides at $t=0$ about the quantity to contract in order to maximize his expected utility given the optimal supply schedule and the joint distribution of $W$ and $\epsilon$.

In particular, the only uncertainty that generators face when they bid their supply schedules $(t=1)$ corresponds to the uncertainty about demand $(\epsilon)$ at the moment of delivery $(t=2)$. Formally, firm $i$ has the fol- 
lowing problem at $t=1$ :

$$
\max _{\hat{S}_{i}(p)} \int_{\underline{p}}^{\bar{p}} \quad U_{i}\left[\hat{S}_{i}(p) p-C_{i}\left(\hat{S}_{i}(p), \vec{W}\right)+\left(P C^{*}-p\right) Q C_{i}^{*}\right] d H_{i}\left(p, \hat{S}_{i}(p)\right)(6.4)
$$

Define $Q C_{i}^{G}$ as the quantity contracted by generator $i$ (chosen at $t=0$ ). Taking the previous assumptions into account, the results of proposition 2.2.3 apply. Therefore, the spot market equilibrium at $t=2$ is given by:

$$
\begin{aligned}
S_{i}^{*} & =\frac{\tilde{D}+Q C_{i}^{G}-\frac{\sum_{j \neq i} Q C_{j}^{G}}{K-1}}{K} \\
p^{c} & =a-\frac{b}{K(K-2)} \sum_{i=1}^{K} Q C_{i}^{G}+\sum_{j=1}^{L} \rho_{j} w_{j}+b \frac{(K-1)}{K(K-2)} \tilde{D}
\end{aligned}
$$

As before, $j \neq i$ corresponds to a index that ranges over all generators other than $i$. Notice that, for given hedging quantities, the risk aversion of the bidder does not influence equations (6.5-6.6). ${ }^{6}$ As explained in chapter 2 , this result follows from the fact that the bidder's optimum in every state of nature is attainable and produces a supply schedule that is a monotonically increasing function of price and realizations of the residual demand. In other words, the bidder has a unique optimal supply for each state of nature related to residual demand.

As discussed in chapter 2, equation (6.6) has the following characteristics. First, the electricity spot price is affected by the state variables in the expected way. Positive shifts in generators' costs and in aggregate demand increase the spot price and negative shifts have the opposite effect. Second, an increase in the retail price decreases aggregate demand and consequently decreases the spot price. The third and most significant aspect of this equation is that players' equilibrium hedge decisions, here represented by the sum of generator's contracted quantities $\sum_{i=1}^{K} Q C_{i t}^{G}$, can

\footnotetext{
${ }^{6}$ We show in the next section that, in equilibrium, risk aversion do affect equations (6.5-6.6) via $\overrightarrow{Q C}^{G}=\left\{Q C_{1}^{G}, Q C_{2}^{G}, \ldots, Q C_{K}^{G}\right\}$.
} 
have a significant role in spot price formation. It means that a large forward position decided at $t=0$ can offset generators' spot market power and affect the spot price at $t=2$. Notice that retailers make no decision at $t=1$ in our model.

\subsection{Hedging decision and equilibrium results}

In the first period $(t=0)$, managers of generators and retailers make the decision of how much electricity to hedge (i.e. they choose $Q C_{i}^{*}$ ). Assume that the utility function $U($.) can be approximated by a quadratic function, yielding a mean-variance utility locally. The maximization problem at $t=$ 0 is then:

$$
\max _{Q C_{i}^{*}} E\left[\pi_{i}^{*}\right]-\frac{\lambda_{i}}{2} \operatorname{Var}\left[\pi_{i}^{*}\right]
$$

Define $\lambda_{i}$ as the firm $i$ 's risk aversion parameter. In order to simplify the results, assume that all the generators have the same risk aversion $\left(\lambda_{i}=\lambda_{G}\right.$ for all generators) and similarly for retailers $\left(\lambda_{i}=\lambda_{R}\right.$ for all retailers). In this case, generators are perfectly symmetric and have the same optimal quantity contracted $\left(Q C_{i}^{G *}=Q C^{G *}\right)$. From equation (6.5-6.6), this symmetry assumption implies that the equilibrium results at $t=1$ change to the following:

$$
\begin{aligned}
S^{*} & =\frac{\tilde{D}}{K} \\
p^{c} & =a-\frac{b}{K-2} Q C^{G *}+\sum_{j=1}^{L} \rho_{j} w_{j}+b \frac{(K-1)}{K(K-2)} \tilde{D}
\end{aligned}
$$

This results from the fact that if $Q C_{i}^{G *}=Q^{G *}$ then $Q C^{G *}-\frac{\sum_{j \neq i} Q C^{G *}}{K-1}=0$ and $\sum_{i=1}^{K} Q C_{i}^{G *}=K Q C^{G *}$. Equations (6.8-6.9) also imply that all generators have the same optimal aggregate profit and operating profit, respec- 
tively defined as $\pi^{* G}$ and $\Upsilon^{*}$ (see definition 6.2.3). Retailers do not participate in the spot market. They maximize their mean-variance function at $t=0$ by choosing their optimal quantity contracted $Q C_{i}^{R}$. Their retail market share is assumed exogenous. That is, retailers optimize their hedging strategies ex-ante at $t=0$ based on both the mean and the variance of their profits $\left(m_{i}\left(p^{R}-p^{c}\right) \tilde{D}\right)$ revealed solely at $t=2$.

Define $\sigma^{2}$ to be the variance of $p^{c}, Q C^{* G}$ the optimal quantity contracted by each symmetric generator and $Q C_{i}^{* R}$ the optimal quantity contracted by retailer $i$.

Proposition 6.3.1. Solving the mean-variance problem for a given hedge price, the generator's and retailer $i$ 's optimal quantities contracted are given by:

$$
\begin{aligned}
Q C^{* G} & =\frac{P C-E\left(p^{c}\right)}{\lambda_{G} \sigma^{2}}+\frac{\operatorname{cov}\left(p^{c}, \Upsilon^{*}\right)}{\sigma^{2}} \\
Q C_{i}^{* R} & =\frac{P C-E\left(p^{c}\right)}{\lambda_{R} \sigma^{2}}+m_{i} \frac{\operatorname{cov}\left(p^{c},\left(p^{R}-p^{c}\right) \tilde{D}\right)}{\sigma^{2}}
\end{aligned}
$$

Proof: appendix D.

The interaction between the equilibrium results (6.10-6.11) and each of the stochastic variables is not simple. State variables and demand fluctuations ambiguously affect the risk exposure of generators and retailers through their impact on clearing prices, costs and demand. For example, the spot price is linearly, positively and additively affected by the stochastic distribution of $\vec{W}$. On the other hand, the uncertainty about cost and demand has nonlinear (and sometimes ambiguous) effect on revenues and costs of generators and retailers. However, since spot prices are positively linked to generation revenue, it is reasonable to expect that $K \operatorname{cov}\left(p^{c}, \Upsilon^{*}\right)$, the covariance between spot prices and generators' operating profits, is positive. For an analogous reason, since spot prices are directly related to retail costs, it is also reasonable to expect that $\operatorname{cov}\left(p^{c},\left(P^{R}-p^{c}\right) \tilde{D}\right)$, the covariance between spot prices and retailers' operating profits, is negative for a given retail price $p^{R}$. That is, generators are expected to have a posi- 
tive risk exposure to spot prices while retailers have a negative risk exposure. We adopt the assumptions that $K \operatorname{cov}\left(p^{c}, \Upsilon^{*}\right)>0$ and $\operatorname{cov}\left(p^{c},\left(P^{R}-\right.\right.$ $\left.\left.p^{c}\right) \tilde{D}\right)<0$. Our numerical exercise (section 6.4) validates this assumption.

From proposition 6.3.1, we observe that, for a given $P C$, the hedging decision depends on two factors. The first is the risk exposure, represented by the price, operating profit covariance relative to the price variance. We describe this component as an operating beta, in which case the generation beta is $\frac{\operatorname{cov}\left(p^{c}, \Upsilon^{*}\right)}{\sigma^{2}}$ and the retail beta is $m_{i} \frac{\operatorname{cov}\left(p^{c},\left(p^{R}-p^{c}\right) \tilde{D}\right)}{\sigma^{2}}$. Notice that the retail beta of an individual retailer depends directly on his market share $m_{i}$. That is, the risk exposure of retailers is proportional to their size (retail market shares).

The second factor is the willingness to profit from forward premium discrepancies. It is represented by the forward premium itself over the cost of assuming this risky position (risk aversion $\lambda_{R}$ or $\lambda_{G}$ times the price variance $\sigma^{2}$ ). It implies that, given $\lambda \mathrm{s}$ and $\sigma^{2}$, an increase in the forward premium $\left(P C-E\left(p^{c}\right)\right)$ raises the expected profit of selling electricity in the forward market $\left(Q C_{i}>0\right)$. That is, ceteris paribus, an increase in the forward premium increases the incentives for assuming a short position (or for reducing a long position) in the forward market.

Proposition 6.3.2. Assume $\operatorname{cov}\left(p^{c}, \Upsilon^{*}\right)>0$ and $\operatorname{cov}\left(p^{c},\left(P^{R}-p^{c}\right) \tilde{D}\right)<0$. From $\sum_{i=1}^{N} Q C_{i}^{*}\left(P C^{*}\right)=0$ (the forward market clearing condition), we have the following equilibrium results:

$$
\begin{aligned}
P C^{*} & =E\left(p^{c}\right)-\frac{K \operatorname{cov}\left(p^{c}, \Upsilon^{*}\right)+\operatorname{cov}\left(p^{c},\left(P^{R}-p^{c}\right) \tilde{D}\right)}{\frac{R}{\lambda_{R}}+\frac{K}{\lambda_{G}}} \\
Q C^{* G} & =\frac{\operatorname{cov}\left(p^{c}, \Upsilon^{*}\right)-\frac{\lambda_{R}}{\lambda_{G}} \frac{\operatorname{cov}\left(p^{c},\left(P^{R}-p^{c}\right) \tilde{D}\right)}{R}}{\sigma^{2}\left(1+\frac{K}{R} \frac{\lambda_{R}}{\lambda_{G}}\right)}>0 \\
Q C_{i}^{* R} & =-\frac{\frac{K}{R} \operatorname{cov}\left(p^{c}, \Upsilon^{*}\right)+\left(\frac{1}{R}-m_{i}\left(\frac{\lambda_{R}}{\lambda_{G}}+\frac{K}{R}\right)\right) \operatorname{cov}\left(p^{c},\left(p^{R}-p^{c}\right) \tilde{D}\right)}{\sigma^{2}\left(\frac{\lambda_{R}}{\lambda_{G}}+\frac{K}{R}\right)}
\end{aligned}
$$


Proof: appendix D.

Assuming the existence of a single forward market, the equilibrium forward price $P C^{*}$ is determined through the forward market clearing condition in which demand equals supply of forward contracts and then $\sum_{i=1}^{N} Q C_{i}^{*}\left(P C^{*}\right)=0$. Equation (6.13) determines the equilibrium quantity contracted $Q C^{* G}$ for generators. Assumptions $\operatorname{cov}\left(p^{c}, \Upsilon^{*}\right)>0$ and $\operatorname{cov}\left(p^{c},\left(P^{R}-p^{c}\right) \tilde{D}\right)<0$ directly imply that $Q C^{* G}>0$. That is, in equilibrium, pure generators sell electricity in the forward market. Equation (6.14) determines the equilibrium quantity contracted $Q C_{i}^{* R}$ for pure retailers $i$. By the forward market clearing condition $\left(\sum_{i}^{R} Q C_{i}^{* R}=-K Q C^{* G}\right)$, the aggregate retailers' quantity contracted is negative $\left(\sum_{i}^{R} Q C_{i}^{* R}<0\right)$. That is, on aggregate, retailers have a long position in the electricity forward market.

We observe from equation (6.12), the sign of the risk premium $\left(P C^{*}-\right.$ $\left.E\left(p^{c}\right)\right)$ is affected by the aggregate operating risk exposure in the market of generators and retailers, where risk exposure to spot prices is the covariance between clearing spot prices and firms' optimal operating profits. Therefore, if the positive risk exposure of generators as a whole more than offsets the negative aggregate risk exposure of retailers, we have a negative forward premium (i.e a forward discount). Otherwise, if the negative risk exposure of retailers predominates, we have a positive forward premium. The magnitude of this forward premium (or discount) is increased in absolute value by the risk aversion of both generators and retailers.

In equilibrium, from equation (6.13), two issues arise. One is that the negative forward premium effect of generators' operating exposure is offset by its positive direct effect on its hedging decision. The second is that the positive effect of retailers' operating risk exposure on the forward premium appears in the equilibrium generator's optimal forward position equation. That is, an increase in each of generators' and retailers' operating risk exposures increases generators' demand for forward contracts. Notice that an increase in the risk aversion of retailers relative to the risk 
aversion of generators $\left(\frac{\lambda_{R}}{\lambda_{G}}\right)$ has an ambiguous effect over the equilibrium quantities contracted by both generators and retailers.

By construction, retailers' contracts mirror the generators' decisions. From equation (6.14), we observe that the net impact of aggregate retailers' risk exposure $\left(\operatorname{cov}\left(p^{c},\left(P^{R}-p^{c}\right) \tilde{D}\right)\right)$ on a particular retailer $i^{\prime}$ s optimal quantity contracted $\left(Q C_{i}^{* R}\right)$ depends on whether the risk premium effect or the direct hedging effect prevails. If his market share $\left(m_{i}\right)$ is sufficiently small, he can even sustain a long forward position (i.e. optimally contract to sell electricity forward).

Proposition 6.3.3. From the equations (6.5-6.14), we have the following markup for all $i=1,2, \ldots, K$ :

$$
E\left(p^{c}-M C_{i}\right)=\frac{b}{(K-2)}\left(\frac{E(\tilde{D})}{K}-\frac{\operatorname{cov}\left(p^{c}, \Upsilon^{*}\right)-\frac{\lambda_{R}}{\lambda_{G}} \frac{\operatorname{cov}\left(p^{c},\left(P^{R}-p^{c}\right) \tilde{D}\right)}{R}}{\sigma^{2}\left(1+\frac{K}{R} \frac{\lambda_{R}}{\lambda_{G}}\right)}\right)(6
$$

Proof: appendix D.

Equation (6.15) shows that the spot price mark-up of a particular generator is decisively reduced by the risk choice of generators as whole at $t=0$. As observed by papers such as Allaz and Villa (1993), Newbery (1998), Green (1999) and Bushnell (2007), exogenous forward contracts can reduce the spot price mark-up. However, here, forward contracts are endogenous. The forward decisions of retailers and gentailers respond to their risk aversion and to the volatility of variables affecting their risk exposure. In absolute values, higher generator or retailer risk exposure raises the volume of contracts and therefore decreases the capability to exert market power. In other words, in electricity markets with a forward market, market power indexes, such as the Lerner index, can capture the risk configuration instead of completely reflecting the generators' potential market power. A Lerner index close to zero $(E(p-M C) \approx 0)$ could be erroneously interpreted as a sign of competitive conduct when it could be a symptom 
of the existence of high risk exposure at the moment when forward contracts were transacted. In this sense, since risk is costly, there would be a trade-off between managing risk (reducing their cost) and more competitive prices (decreasing generators' operating revenue).

Market power is typically assessed with respect to the spot market. Holding everything else constant, increases in the spot price mark-up ( $p-$ $M C$ ) have a negative welfare effect. For this reason, competition authorities are frequently concerned about the existence of price mark-ups. Consider that $\epsilon$ is negligible since the time auctions take place $t=1$ and the time of spot market clearing $t=2$ are frequently very close. The forward market remaining in equation (6.15) shows the need in the market power assessment to control for risks associated with the state variables $W$ in order to separate risk management from potential anti-competitive behavior. Since uncertainty can also produce negative impacts on welfare and generators face a trade-off between hedging and sacrificing market power, the equilibrium impact of their choices on welfare should not be considered in isolation. More explicit analysis is helped by a numerical example.

\subsection{Numerical exercise}

To illustrate implications of cost and demand volatility for our equilibrium hedging results we conduct a series of simulations based on calibrated parameters as approximations to actual values. The purpose is to explore the role of supply-side volatility, as well as demand volatility, in determining the supply and demand for hedges. We also address the effect of changes in both volatilities on the forward premium and on expected market power. Comparative static results will be explored by varying the parameters. 


\subsubsection{Calibration Methodology}

We assume two random state variables: one state variable shifting costs $\tilde{w}_{2 t}$ and the other being the demand $\tilde{D}_{t}$. Equation (6.9) states that:

$$
\begin{aligned}
p_{t}^{c} & =\psi+\rho \tilde{w}_{2 t}+\beta \tilde{D} \\
\psi & =a-\frac{b}{K-2} Q C^{G *} \\
\beta & =b \frac{(K-1)}{K(K-2)}
\end{aligned}
$$

Our calibration strategy has two basic steps. First, we regress the spot price against historical series of the state variables which gives us a fitted equation for the spot price at $t$.

$$
\hat{p}_{t}^{c}=\hat{\psi}+\hat{\rho} \tilde{w}_{2 t}+\hat{\beta} \tilde{D}_{t}
$$

Notice that endogeneity is not a problem in the estimation of equation (6.17), since we are only interested in the equilibrium relationship between spot price and state variables. That is, we do not need to estimate the structural functions of demand and supply since the identification of the relevant parameters is done by our theoretical model. Rearranging the coefficients we have:

$$
\begin{aligned}
\hat{b} & =\frac{K(K-2)}{K-1} \hat{\beta} \\
\hat{a} & =\hat{\psi}+\hat{\beta} \frac{K}{K-1} Q C^{* G}
\end{aligned}
$$

The equilibrium quantity contracted $Q C^{* G}$ is almost never an observed variable. Define $\hat{P C}$ as the actual observed future/forward price. Assume that the risk aversion of retailers and generators is the same $\left(\lambda_{G}=\lambda_{R}=\right.$ $\lambda)$ and that the expected retail margin $p^{R}-E\left(p^{c}\right)$ is fixed and equal to 
$\nu .^{7}$ Substituting (6.17-6.19) in equations (6.8-6.9) and (2.2), we have the following stochastic variables:

$$
\begin{aligned}
p^{c} & =\hat{a}+\hat{\rho} \tilde{w}_{2 t}+\hat{\beta}\left(\tilde{D}_{t}-\frac{Q C^{* G}}{K-1}\right) \\
S^{*} & =\frac{\tilde{D}}{K} \\
C^{*} & =\left(\frac{K-2}{2 K(K-1)} \hat{\beta}\right)(\tilde{D})^{2}+\left(\hat{\psi}+\frac{\hat{\beta}}{K-1} Q C^{* G}+\hat{\rho} \tilde{w}_{2 t}\right) \frac{\tilde{D}}{K}+F(62 \\
\Upsilon_{*} & =S^{*} p^{c}-C^{*} \\
\pi^{* G} & =\Upsilon *+\left(P C^{*}-p^{c}\right) Q C^{* G}
\end{aligned}
$$

From equations (6.12-6.14), we need to calculate the covariances to obtain $P C^{*}$ and $Q C^{* G}$. Therefore, we need to assume a probability distribution for the stochastic variables we observe $\left(w_{2}\right.$ and $\left.\tilde{D}\right)$ and estimate its parameters. For simplicity, assume that the state variables follow a multivariate normal distribution $\left(W=\left(w_{2 t}, \tilde{D}_{t}\right) \sim N I D\left(\left(E\left(w_{2 t}\right), E\left(\tilde{D}_{t}\right)\right), \Sigma\right)\right)$. Consider the historical means and unconditional variance to be reasonable estimators. Substitute equations (6.20-6.23) in equations (6.12-6.14). For a given $\lambda$, we have the following optimal quantity contracted by generators:

$$
Q C^{* G}=\frac{\operatorname{cov}\left(p^{c}, \Upsilon^{*}\right)-\frac{\operatorname{Cov}\left(p^{c},\left(P^{R}-p^{c}\right) \tilde{D}\right)}{R}}{\hat{\sigma}^{2}\left(1+\frac{K}{R}\right)}
$$

In order to calibrate the risk aversion, choose the $\hat{\lambda}$ that equates the equilibrium forward price $P C^{*}$ to an observed forward/future price $\hat{P C}$. Given

\footnotetext{
${ }^{7}$ This is not an unrealistic assumption since retail prices are relatively fixed in comparison with the volatile spot prices. At the same time, it is reasonable to suppose that, at $t=0, p^{R}$ is greater than the expected spot price $E\left(p^{c}\right)$. Otherwise, retailers would not have reasons to be in the business. The assumption of a fixed expected retail margin simplifies our results.
} 
equation (6.12), $\hat{\lambda}$ solves the following:

$$
\hat{P C}-E\left(p^{c}\right)=-\hat{\lambda} \frac{K \operatorname{cov}\left(p^{c}, \Upsilon^{*}\right)+\operatorname{cov}\left(p^{c},\left(P^{R}-p^{c}\right) \tilde{D}\right)}{R+K}
$$

From equations (6.8-6.15), we notice that all the estimates are unconditional (means, variances and covariances). When deciding their hedging strategies at $t=0$, firms only know the unconditional distribution of the state variables.

\subsubsection{Illustrative example: New Zealand}

The characteristics of NZEM were discussed in section 4.3. Briefly, NZEM is predominantly hydro-based. It is composed of five major generators $(K=5)$ and five retailers $(R=5)$. The generators are assumed to have a symmetric cost structure for simplification to the model. Here we use a numerical exercise to illustrate how hedge/demand ratio and market power are affected by demand and cost volatilities if either vertical integration is not considered, or vertical separation is implemented or VI mimics hedging. The last section of this chapter relaxes this hypothesis and analyzes the implications of vertical integration.

Consider again the Haywards node spot price $p^{c}$, the aggregate market demand $D$ and NZ hydro lake inflows $\left(w_{2}\right)$. The variables, and their sources, are the same as explained in section 4.3. We use monthly frequency data from $04 / 2004$ to $06 / 2010$. The monthly series are built from an arithmetic average of the daily series. That is, we assume that the interval between the contract decision $(t=0)$ and the spot market clearing $(t=2)$ is one month. The results of applying an ordinary least square regression (OLS) to equation (6.17) are represented in Table 6.1: Table 6.1 shows that the parameters have the expected sign. The parameter $\hat{\rho}$ indicates that the water inflows have a statistically significant effect on monthly prices. However the high p-value of the intercept and demand imply that they are not statistically different from zero. For the purposes 
Table 6.1: Results of the OLS estimation of equation (6.17)

\begin{tabular}{lcc}
\hline Parameters & Coefficients & p-value \\
\hline$\hat{\psi}$ & 88.10 & 0.35 \\
$\hat{\rho}$ & -0.06 & 0.00 \\
$\hat{\beta}$ & 0.66 & 0.43 \\
\hline
\end{tabular}

of this numerical illustration, we use these point estimates and ignore the confidence interval.

To complete our calibration we need to estimate the one-month ahead forward premium $\left(\hat{P C}-E\left(p^{c}\right)\right)$. Note that $E\left(p^{c}\right)$ is unconditional in equation (6.12). Specifically, we use the average forward premium in the interval between $04 / 2004$ and 06/2010.

We choose the month-ahead Haywards forward prices as proxies for $P C$. Unfortunately, the month-ahead Haywards contract is not traded each month. There are some months where only forward contracts for quarterly delivery are traded for initiate delivering at the month ahead. To be coherent in terms of the the length of the forward contracts, we use exclusively month-ahead contracts. This requires that we calculate our forward premium average from an irregular series since there are missing observations in the month-ahead Haywards forward price series. In particular, we build our forward price series by considering the Haywards forward price at the first trading day of a specific month $t$ for delivering electricity at the following month $t+1$.

Given the information available at $t$, we use an ordinary least square (OLS) regression to calculate the unconditional expected spot price (one month ahead). In particular, we calculate $E^{t+1}\left(p_{t}^{c}\right)$ by estimating equation (6.16) using the information available in each month. Specifically, we use the demand and water inflows data from $01 / 1997$ to $t$ to estimate the unconditional expected spot price at $t+1 .^{8}$ The average of the 65 forward premium observations in the interval is $2.6 \%$.

\footnotetext{
${ }^{8}$ For simplification, issues related to seasonality are not considered in this formulation.
} 
Consider the historical mean and the unconditional variance-covariance as proxies to our distributional parameters. Approximately, we have $E(\tilde{D})=$ 102.62, $E\left(w_{2}\right)=1422.86, \sigma_{D}=6.60, \sigma_{2}=353.83$ and $\operatorname{corr}\left(w_{2}, \tilde{D}\right)=-0.2515$. From equation (6.26), we numerically obtain $\lambda=0.00045$. The $\lambda$ s and the remaining calibrated parameters are shown in Table 6.2.

Table 6.2: Calibrated Parameters for the model outlined in equations (6.126.15)

\begin{tabular}{lccc}
\hline$a$ & 97.27 & $\rho$ & -0.06 \\
$b$ & 2.46 & $\lambda_{G}$ & 0.00045 \\
$\operatorname{corr}\left(w_{2}, \tilde{D}\right)$ & -0.2515 & $\lambda_{R}$ & 0.00045 \\
$\bar{w}_{2}$ & 1422.86 & $\sigma_{D}$ & 6.60 \\
$\bar{D}$ & 102.62 & $\sigma_{2}$ & 353.83 \\
$K$ & 5 & $R$ & 5 \\
\hline
\end{tabular}

\subsubsection{The Role of Cost and Demand Volatilities}

We assume that volatilities of $w_{2}$ and $\tilde{D}$ can range from half the value of the historical standard deviations shown in Table 6.2 to three times these values. Figure 6.2 shows that, in this situation, the NZEM forward price bias $\frac{P C^{*}-E\left(p^{c}\right)}{E\left(p^{c}\right)}$ is positively affected by both the hydro inflow volatility (cost shifter) and the demand volatility. Bessembinder and Lemmon (2002) observe that, for a deterministic quadratic cost function, an increase in the demand volatility decreases the forward price bias. The authors show that the opposite happens for an exponential cost function of higher degree. In their model, an increase in the non-linearity of costs increases the skewness of the spot price distribution which accounts for higher bias. Here, with uncertainty in costs, we show that it is possible to have positive bias even with a quadratic cost function. Moreover, the forward price bias can be more responsive to increases in the cost volatility than in demand volatility.

This means that an increase in hydro inflow volatility increases the re- 
Figure 6.2: Bias in forward price as function of estimated parameters as per Table 6.2. Model used is outlined by equations (6.12-6.15)

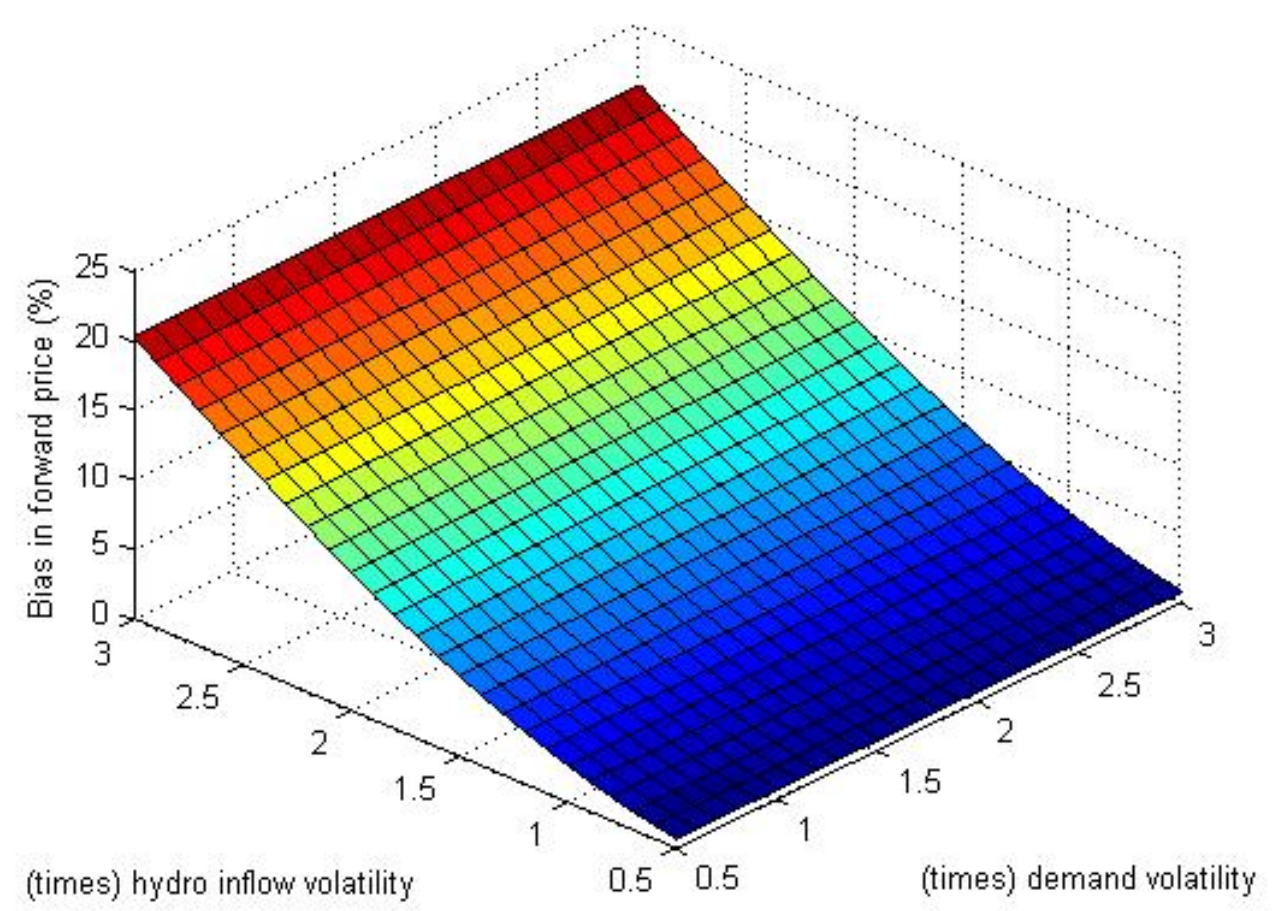

tailers' operating risk exposure more than it increases the generators' operating risk exposure. In other words, the positive effect of cost volatility on retailers' operating risk exposure dominates the also positive effect on generators' risk exposure, which produces a pronounced positive impact on forward bias. On the other hand, the demand volatility effects on generators' and retailers' risk exposures are of equivalent magnitude which makes their net impact on the forward bias almost offset. Particularly, the sensitivity of the forward price bias to demand volatility depends on the parameter $\rho$ (sensitiveness of marginal costs to $w_{2}$ ). The sign of the demand volatility effect on forward bias can even change for sufficiently high values of $\rho$. Figure 6.3 shows that the impact of cost volatility and demand volatility on the generator's hedge ratio $\left(\frac{Q C^{* G}}{E\left(S^{*}\right)}\right)$ can have opposite 
Figure 6.3: Hedging as function of estimated parameters as per Table 6.2. Model used is outlined by equations (6.12-6.15)

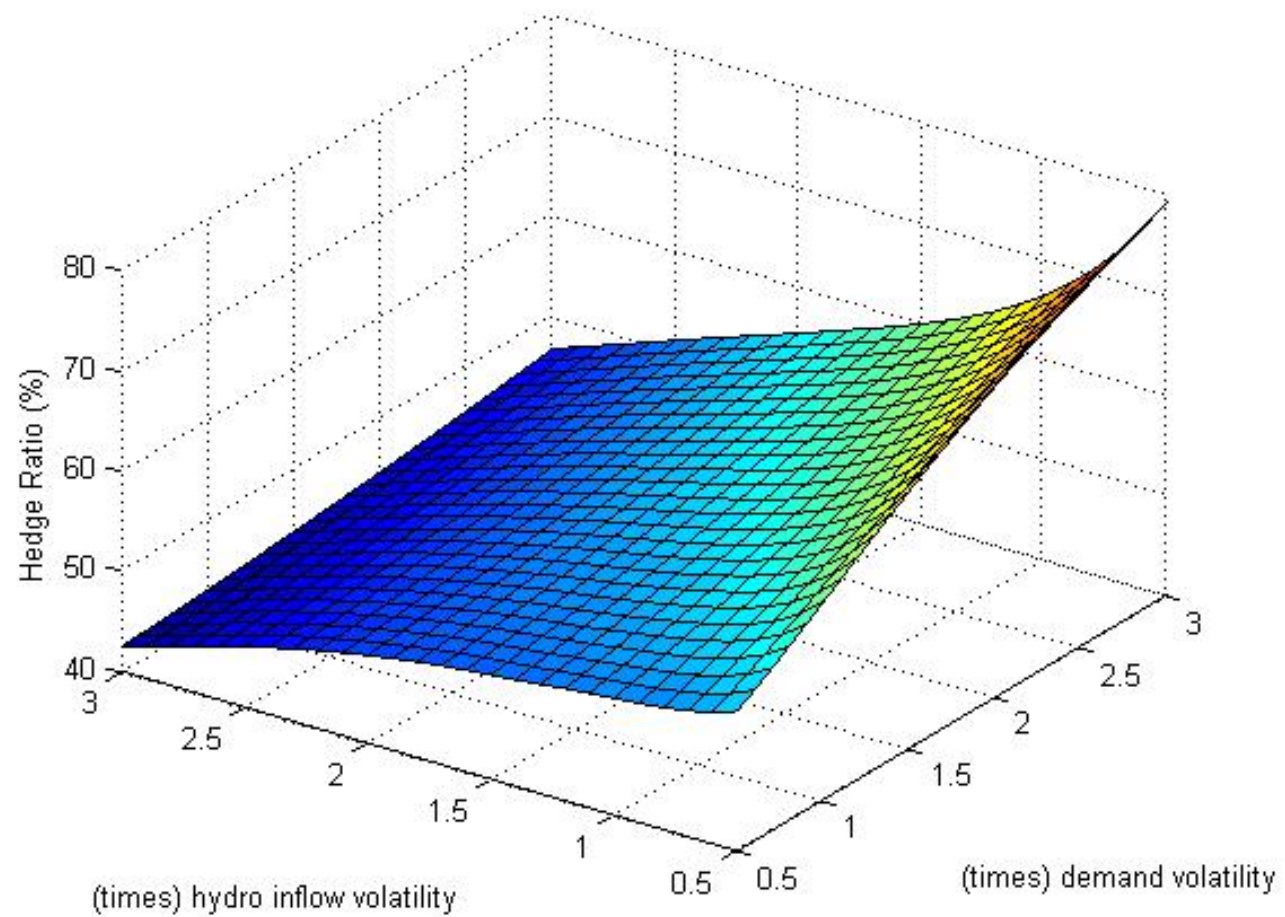

signs. As illustrated above, an increase in the demand volatility increases the generators' relative demand for hedging protection and an increase in cost volatility affects their optimal hedge ratio negatively. As discussed in the previous section, ceteris paribus, optimal hedging reacts in response to the effect of the state variables on both generators and retailers' systematic profit risks and on the forward premium. Using our calibrated parameters, the cost and demand volatility effects on generators' beta is mostly responsible for variations in optimal hedging since their effect on retailers' beta is almost negligible. Notice that, in this case, the negative effect of cost volatility on optimal hedging ratios is greater in a high demand volatility scenario. The reason is that in a high demand volatility 
Figure 6.4: Market power as function of estimated parameters as per Table 6.2. Model used is outlined by equations (6.12-6.15)

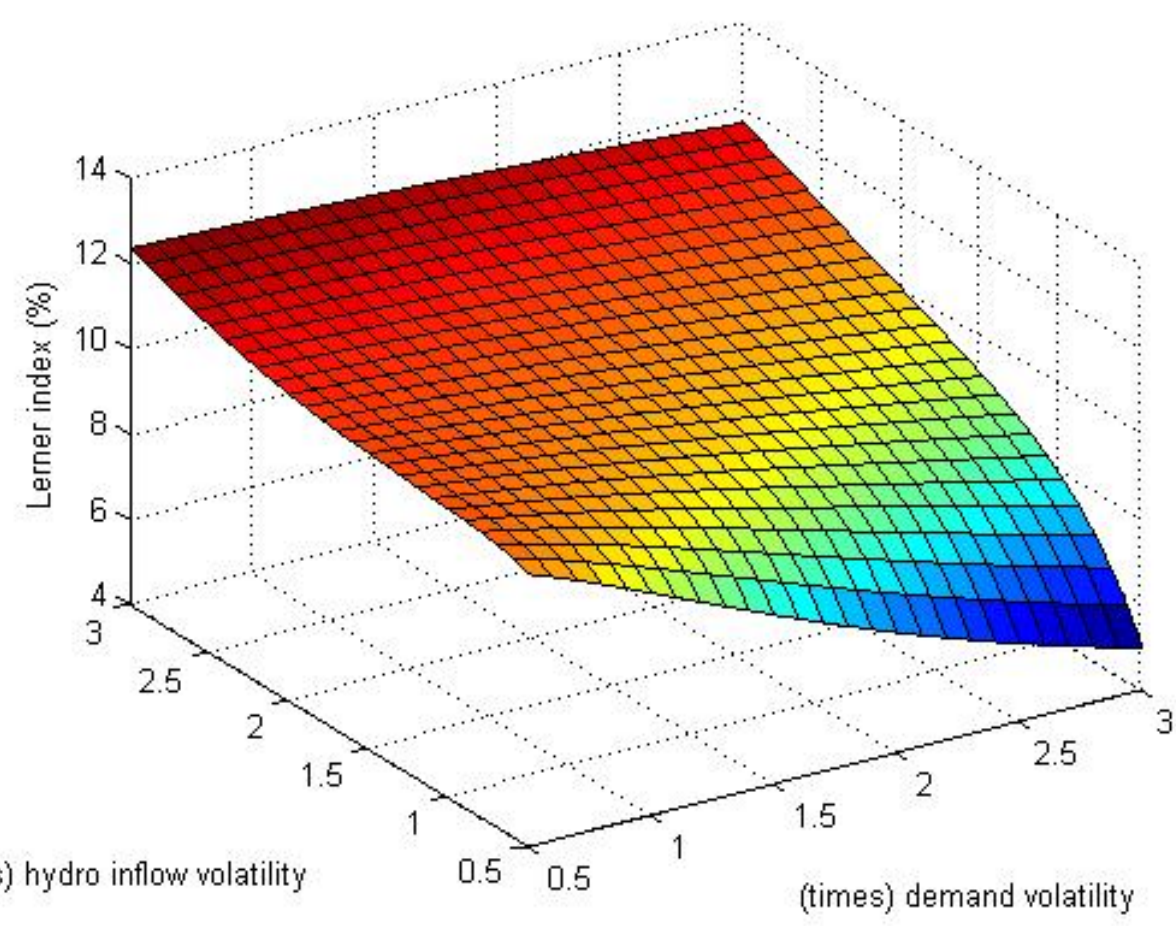

situation, increases in $\sigma_{2}$ lead to decreases in generators' operating beta. In other words, bigger hydro inflow volatility augments the price volatility more than it increases the covariance between price and generators' operating profit. An increase in $\rho$ magnifies this effect. Decreases in $\rho$ can even lead to a positive relationship between hydro inflow volatility and optimal hedge ratios given the calibrated demand volatility.

Lastly, we notice from Figure 6.4 the importance of risk management over the generator's expected market power, here represented by an adjusted Lerner index $\left(\frac{E\left(p^{c}-M C^{*}\right)}{E\left(p^{c}\right)}\right)$. Basically, the market power results mirror the optimal hedging determinants. In the situation of high water outflow volatility we have higher market power. On the other hand, an increase in the demand volatility diminishes the Lerner index. In other words, the 
capability to exert market power in an oligopolistic framework can be constrained or stimulated by a particular risk environment. Finally, as observed, cost-side and demand-side risks can have different roles and may have different effects.

\subsection{Vertical integration}

This section analyzes the impact of vertical integration over the empirical results previously discussed. We focus on the case of fully vertically integrated markets such as New Zealand, Spain or Germany. In particular, we show that mismatches in generation market share and retail market share justify the existence of forward markets. However, important results that hold for vertically separated markets are not valid under the full VI assumption.

Consider now that generator $i$ can participate of the retail market and has a market share of $m_{i} \geq 0$, and define the sum of the market shares of generators (gentailers) in the retail market to be $\sum_{i=1}^{K} m_{i}=m^{I}$.

The generator/gentailer $i$ 's ex-post profit upon the realization of the market clearing price at $t=2$ is:

$$
\pi_{i}^{I}=S_{i} p^{c}-C_{i}\left(S_{i}, W\right)+\left(p^{R}-p^{c}\right) m_{i} \tilde{D}+\left(P C-p^{c}\right) Q C_{i}
$$

In this case, the maximization problem is the following:

$$
\begin{aligned}
\max _{Q C_{i}, \hat{S}_{i}} E_{W, \epsilon}\left[U_{i}\left(\pi_{i}^{I}\right)\right] & =\max _{Q C_{i}} E_{W}\left[\max _{\hat{S}_{i}} E_{\epsilon \mid W}\left[U_{i}\left(\pi_{i}^{I}\right)\right]\right] \\
& =\max _{Q C_{i}} E_{W, \epsilon}\left[U_{i}\left(\pi_{i}^{I}\left(\hat{S}_{i}^{*}\right)\right)\right]
\end{aligned}
$$

The maximization problem of pure retailers is the same as given by definition 6.2.5. Again, at at $t=0$ firms decide their forward contracts, at $t=1$, firms decide their supply schedules and at $t=2$ all the stochastic variables are revealed. If $m_{i}=0$, firm $i$ is a pure retailer. 
Assume that all the generators and gentailers have the same risk aversion $\left(\lambda_{I}\right)$. Pure retailers have risk aversion equal to $\lambda_{R}$

Suppose that the definitions and propositions of model II described in section 3.4 hold. That is, there is a symmetric Bayesian-Nash equilibrium at $t=2$ where the equilibrium supply and the clearing wholesale spot price are the following:

$$
\begin{aligned}
p_{t}^{c} & =\frac{1}{\sum_{i=1}^{K} \alpha_{i}}\left(\tilde{D}_{t}-\sum_{i=1}^{K} \psi_{i}-\sum_{i=1}^{K} \beta_{i} Q C_{i t}^{I}-\sum_{i=1}^{K} \sum_{j=1}^{L} \delta_{i j} w_{j}\right) \\
S_{i}^{*} & =\frac{K-2}{b(K-1)}\left(p_{t}^{c}-a-\sum_{j=1}^{L} \rho_{j} w_{j t}\right)+\frac{Q C_{i t}^{I}+m_{i} D_{t}}{K-1}
\end{aligned}
$$

, where

$$
\begin{aligned}
\psi_{i} & =\frac{m_{i} \sum_{j \neq i} \psi_{j}+m_{i} \sum_{j \neq i} \beta_{j} Q C_{j}-\sum_{j \neq i} \alpha_{j} a}{1-m_{i}+\sum_{j \neq i} \alpha_{j} b} \\
\alpha_{i} & =\frac{\left(1+m_{i}\right) \sum_{j \neq i} \alpha_{j}}{1-m_{i}+\sum_{j \neq i} \alpha_{j} b} \\
\beta_{i} & =\frac{1}{1-m_{i}+\sum_{j \neq i} \alpha_{j} b} \\
\delta_{i} & =\frac{m_{i} \sum_{j \neq i} \sum_{l=1}^{L} \delta_{j l}-\sum_{j \neq i} \sum_{l=1}^{L} \alpha_{j} \rho_{l}}{1-m_{i}+\sum_{j \neq i} \alpha_{j} b}
\end{aligned}
$$

The equations above state the equilibrium spot price and the equilibrium generator $i$ 's supply at $t=2$ under the possibility of vertical integration.

Consider now a fully integrated market such as NZEM, where $m_{I} \approx 1$. Define $\overrightarrow{Q C}=\left\{Q C_{1}, Q C_{2}, \ldots, Q C_{I}\right\}$ as the vector of the quantities contracted by each gentailer in the market. For all gentailers, we approximate $U\left(\pi_{i}^{I}\right)$ by the mean variance function

$$
F\left(\pi_{i}^{I}\right)=E\left(\pi_{i}^{I}\right)-\frac{\lambda_{I}}{2} \operatorname{var}\left(\pi_{i}^{I}\right)
$$


where $\pi_{i}^{I, R}$ is the profit function of gentailer $i$ evaluated at $\left(p^{c}, S^{*}\left(p^{c}\right)\right)$, which is given by equations (6.28-6.29). Gentailer $i$ 's optimal quantities contracted $\left(Q C_{i}^{*}(P C)\right)$ maximize the objective function (6.34), given all his rivals' optimal quantities contracted $\left(Q C_{j}^{*}(P C), \forall j \neq i\right)$. Vector $Q \vec{C}^{*}=$ $\left\{Q C_{1}^{*}, Q C_{2}^{*}, \ldots, Q C_{I}^{*}\right\}$ represents the optimal quantities contracted by all gentailers in the market. The forward price $P C^{*}$ is given by the price that clears the forward market $\left(\sum_{i}^{I} Q C_{i}^{*}\left(P C^{*}\right)=0\right)$. The retail price $p^{R}$ is taken to be exogenous. We haven't obtained a closed-form solution equivalent to (6.12-6.14) for this vertical integration extension. The problem is solved numerically.

We examine the hedging contracting effects of vertical integration (VI) by means of a calibrated model. The same calibrated model is used to analyze how vertical integration affects forward premium and spot market power.

\subsubsection{Numerical results}

We calibrate this VI model with parameters of Table 6.2. We adjust the 2008 NZEM retail market shares, given by Table 6.2, in order to implement the assumption of a fully vertically integrated market $\left(m_{I}=1\right)$. Table 6.3 illustrate the retail market share adjustment. Figure 6.5 shows that the effects

Table 6.3: Adjusted retail market shares

\begin{tabular}{lcc}
\hline Company & 2008 & 2008 adjusted \\
\hline Contact Energy & $27 \%$ & $29 \%$ \\
Genesis Energy & $25 \%$ & $27 \%$ \\
Meridian Energy & $12 \%$ & $12 \%$ \\
Mighty River Power / Mercury Energy & $19 \%$ & $20 \%$ \\
Trust Power & $11 \%$ & $12 \%$ \\
\hline Total & $94 \%$ & $100 \%$ \\
\hline
\end{tabular}

of the hydro inflow and demand volatilities on the forward price bias (relative forward premium) have the same sign as in the vertically separated 
case. Changes in the demand volatility $\sigma_{D}$ do not alter the forward price bias significantly and increases in the hydro inflow volatility $\sigma_{2}$ increases the forward premium. The reasons have been outlined above. An increase in hydro inflow volatility increases the retailers' operating risk exposure more than it increases the generators' operating risk exposure. In fact, the magnitude of the impact of the cost shifter volatility on the forward premium is bigger in the VI case. That is, given all the assumptions of this empirical exercise, the positive effect of cost volatility on the retail operating risk exposure relative to its effect on the generation risk exposure is more pronounced than in the vertically separated case. In the full VI

Figure 6.5: Bias in forward price as function of estimated parameters as per Tables 6.2-6.3. Model used is outlined by equations (6.27-6.34)

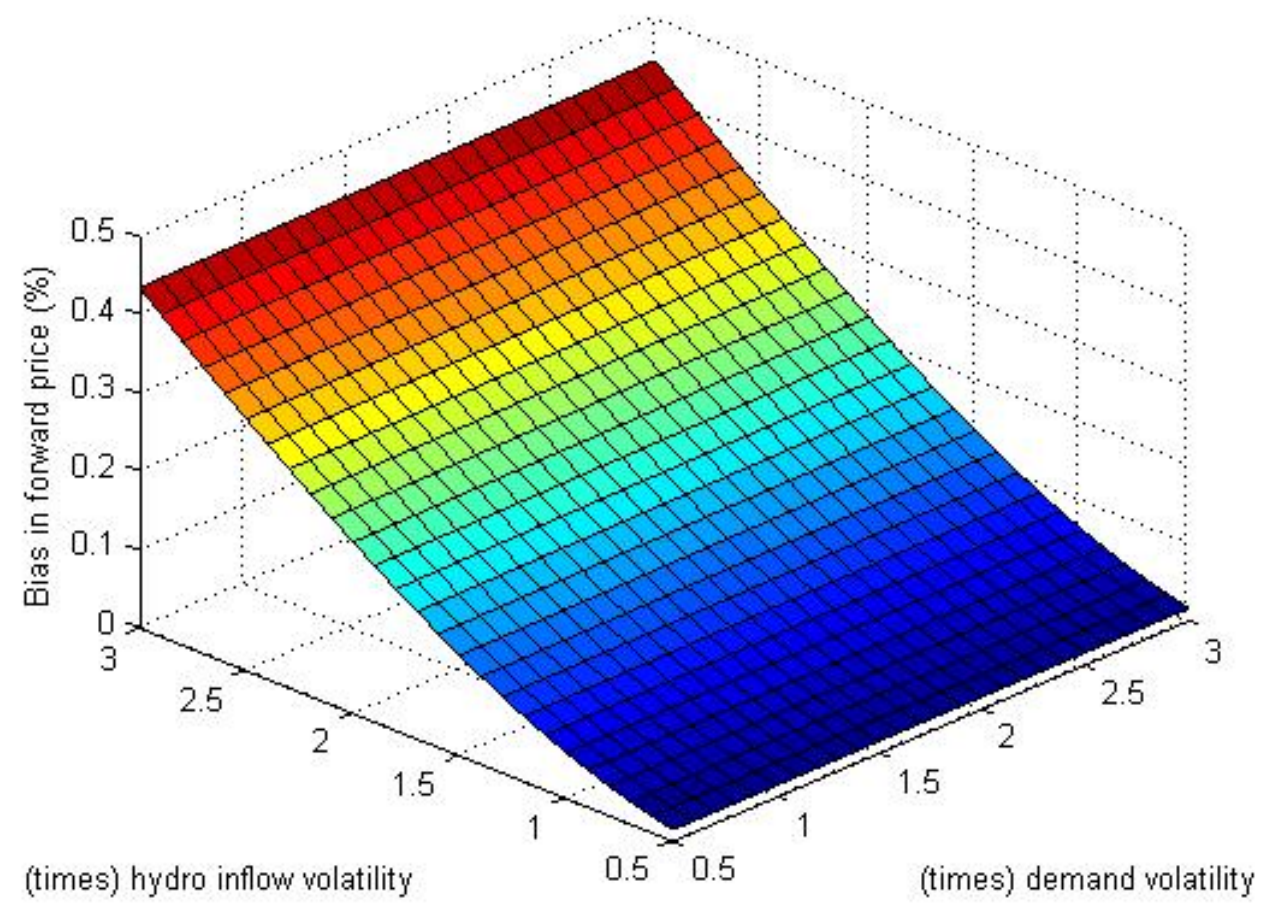

scenario, the gentailers' forward transaction in absolute terms over their production is around $30 \%$. Under complete vertical separation, the gen- 
erators and retailers have a hedge ratio of 54\%. ${ }^{9}$ That is, fully vertically integrated markets can have a reasonable level of forward transactions. In such markets, where there are gentailers with different participation in the generation and retail markets, we have net generators and net retailers that trade with each other.

Figure 6.6 shows that the relevance of volatility to the hedge ratio is quite different from the exercise where generators and retailers are different firms. Both demand and cost volatilities have only marginal effects on the hedge ratio under full vertical separation. The reason is that, unlike pure retailers and pure generators, gentailers internalize effects of opposite signs caused by these volatilities by their vertical integration. Therefore, the aggregate impact of these volatilities on the industry average hedge ratio is considerably attenuated. Lastly, as we discussed in chapter 4 , spot prices are not significantly affected by hedge contracts in an electricity market solely composed of gentailers, regardless of the contract position of individual gentailers. As far as contracts do not affect expected spot prices, they are not affected by demand or cost volatilities as well. As shown by Figure 6.7, this means that the adjusted Lerner index of fully vertically integrated markets is not affected by either demand or hydro inflows volatility. Figure 6.7 also shows that, as occurs in Model I, the average market power is close to zero. The intuition is best expressed by equation (3.4). In a fully vertically integrated market, most of the aggregate demand is hedged through vertical integration and there is no markup of spot prices over the firms' average marginal costs. In summary, chapter 6 uses a three period equilibrium model to analyze hedging decisions in electricity markets. Firstly, the model shows that demand-side and supply-side volatilities have an important role in explaining hedge size. The sign and magnitude of their effects on forward prices strongly depend on the relative risk exposure of generators and retailers. Secondly, it shows that spot price mark-ups can be influenced by risk in electricity

\footnotetext{
${ }^{9}$ Some gentailers are net retailers and have negative optimal quantity contracted $Q C^{*}$
} 
Figure 6.6: Hedging as function of estimated parameters as per Tables 6.26.3. Model used is outlined by equations (6.27-6.34)

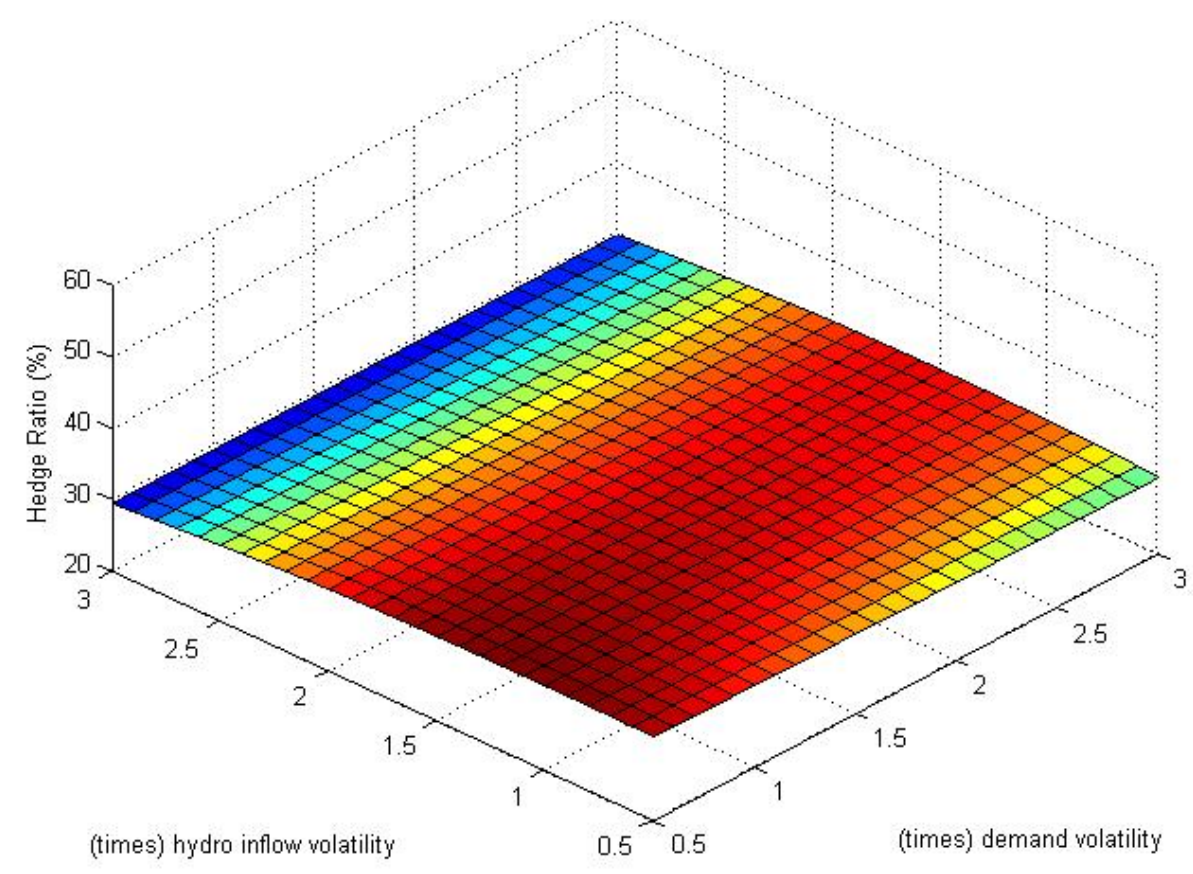

markets which are not entirely hedged. That is, market power measures such as the Lerner index must be controlled for risk, otherwise, they can reflect the exogenous risk environment instead of oligopolistic conduct. Lastly, our empirical exercise shows that demand-side and supply-side volatilities play a lesser role in explaining either the hedge ratio or market power in fully vertically integrated markets such as the NZEM. In fact, as occurs in model I of chapter 4 , the average mark-up is equal zero. The signs of the effect of both volatilities on the forward price remain the same in the vertically separated and in the fully vertically integrated cases. 
Figure 6.7: Market power as function of estimated parameters as per Tables 6.2-6.3. Model used is outlined by equations (6.27-6.34)

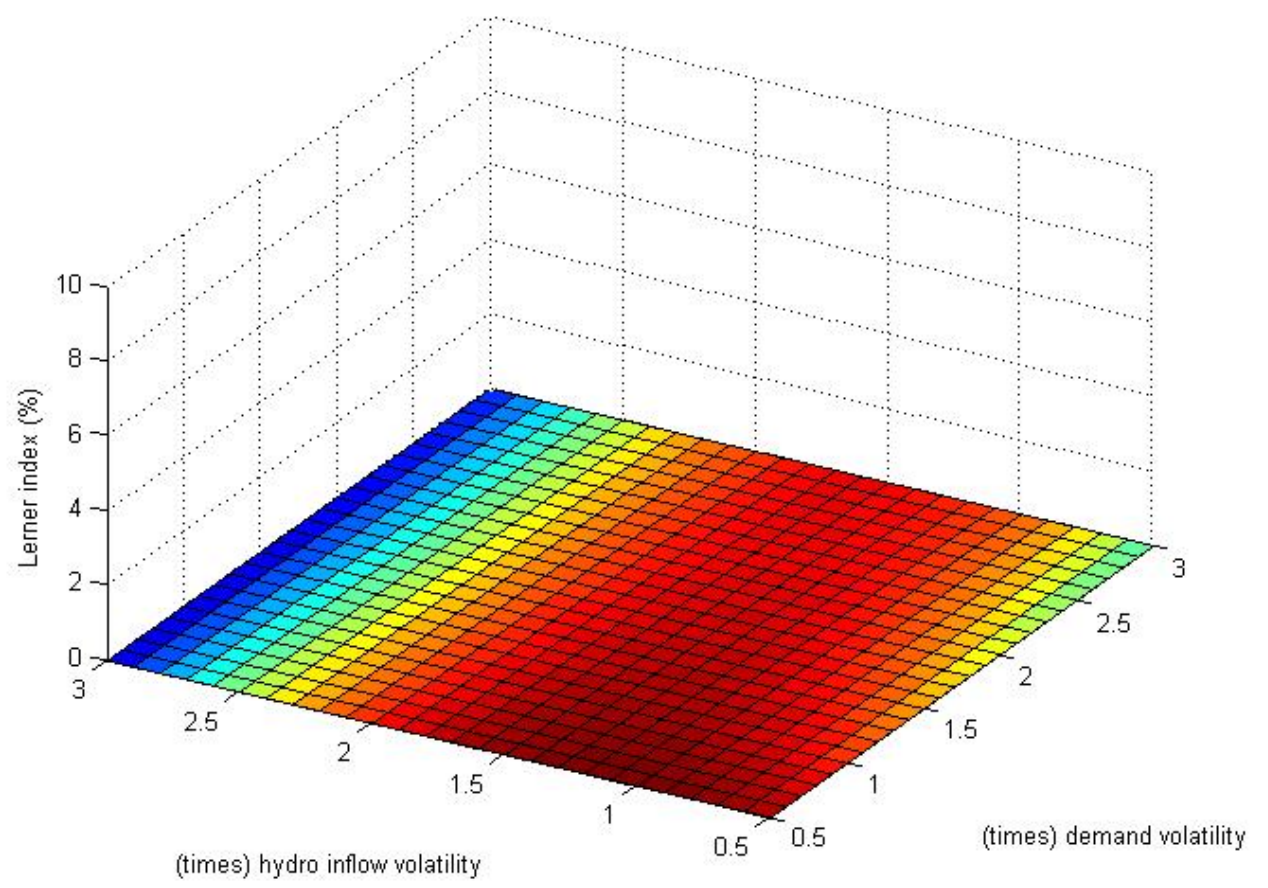




\section{Chapter 7}

\section{Conclusion}

This thesis proposes a new framework to jointly analyze electricity spot market and hedging decisions in an oligopolistic setup. Chapters 2 and 3 develop the spot market equilibrium models which form the basis of the thesis. We find that, when exogenous, both quantity of electricity hedged by contract and vertical integration decrease the equilibrium spot price. Chapter 5 uses a hybrid approach and shows that market structure can affect the generators decision to vertically integrate under uncertain demand. Chapter 4 considers uncertainty in costs and demand and shows that concentration in the spot market, for a given hedge quantum, can increase forward prices and affect the slope of the forward curve. Our empirical results indicate that the model fits the New Zealand electricity market well. This evidence that market structure and hedging decisions are closely connected is further explored in chapter 6 .

Chapter 6 starts from the premise that electricity markets are incomplete and that therefore preferences matter. A three period equilibrium model for the spot and forward markets, where hedging occurs prior to the submission of supply curves, is developed. This model takes into account demand-side and supply-side uncertainties. Most importantly, the spot price, forward price, spot supply and hedging quantities are endogenous. We find that when hedging is endogenous, hedging quantities are 
affected by spot market parameters, but market power is itself mitigated in the hedging choice. An ex-ante positive mark-up in the spot market is implied by the risk environment, but the mark-up is less than it would be in the absence of hedging. This is a clear indication that policy makers should not dissociate market power from risk in their social welfare considerations.

Other important issue in the thesis refers to vertical integration. There is no such thing as fully hedged electricity markets. If electricity firms always know exactly the state of the world at the time of delivery, spot markets would not be necessary. Taking this into account, we show in chapter 6 that highly hedged markets, either by forward contracts or vertical integration, have the average mark-up close to zero. We also show that forward markets can coexist with highly vertically integrated economies if the firms have different market shares in the retail and generation markets (i.e. existence of net retailers and net generators). The New Zealand market is a typical example of such situation.

There are several possible extensions and improvements to this thesis. First, each of the two models of chapter 3 has a single source of uncertainty: rivals forward positions in Model I and/or demand in Model II. An important and challenging theoretical extension would be assuming both sources of uncertainty simultaneously in the vertical integration framework and finding tractable results. This would constitute a generalization of Hortacsu and Puller (2008) for VI markets. Second, fixed retail prices can be a rather strong assumption for extended periods of time. The analysis of chapter 5 about investment in vertical integration would benefit extensively if either retail prices are adjustable or supply-side uncertainty is considered. Third, the same model assumes that the retail expansion is exogenous. An interesting extension would be incorporating strategic issues related to the investment decision and considering the increase in retail participation as an endogenous decision. This would increase the generality (and the complexity) of the problem considerably. 
The model of chapter 6 has a very flexible framework that allows for at least two important extensions. Despite being substitutes to some extent, forward contracts and vertical integration are hedging instruments with different characteristics. In practice, retail contracts adjust more quickly than forward contracts to shocks in demand and prices. Our framework assumes exogenous vertical integration, however the model could be extended to include the decision of vertical integration and the retail market clearing. That is, at the time of the hedging decision, retailers/gentailers would be able to choose their retail market share and retail prices would be the product of the equilibrium in the retail market (also endogenous). This could answer important questions about the determinants of vertical integration in a more general equilibrium setup and also about how retail prices relate to forward and spot prices in equilibrium. Of course, relaxing the hypothesis of symmetry could achieve additional insights at the cost of less tractability. In chapter 6 we also develop an illustrative example based on calibrated parameters. An immediate empirical extension would be to use a more robust econometric framework to test the model. Since the model is mostly based on the moments of stochastic variables (expectations, variances and covariances), the generalized method of the moments (GMM) could be a first candidate for estimating the parameters of our model.

The importance of our results is not strictly theoretical. The hybrid partial equilibrium frameworks of chapters 4 and 5 can be used for policy makers to analyze uncommon implications of changes in the spot market structure. Ceteris paribus, they produce insights about how concentration affects the forward curve, which is an important (and frequently underestimated) reference for decisions of electricity firms. Recall that a significant amount of transactions in electricity markets do not happen in the spot market: even though there may be a compulsory pool. They can also answer questions about the relationship between the spot market structure (e.g. concentration or hedge ratios) and the optimal timing of investments. 
For example, everything else constant, does an increase in the number of firms postpone or encourage investments? Most importantly, the framework of chapter 6 should be taken into account for policy makers. The conclusion that market power and risk are closely connected in equilibrium is, to our knowledge, a new result. A welfare analysis within this framework could achieve promising insights for competition authorities, for example. Our results indicate that electricity generators, in equilibrium, sacrifice market power to protect themselves against both supplyside and demand-side risk. Given that it is socially beneficial to manage risk, it would be interesting to analyze the effects of relaxing symmetry and the exogeneity of vertical integration and retail prices on our results. A reasonable question left for future research is: is there a trade-off between risk and market power in term of social welfare? If the answer is positive, the concern about increases in electricity market power should be put in perspective. They could just reflect a welfare optimal option for risk reduction. 


\section{Bibliography}

Aghion, P. and P. Bolton (1987), Contracts as a barrier to entry, The American economic review $77,388-401$.

Allaz, B. and J-L. Villa (1993), Cournot competition, forward Markets and efficiency, Journal of Economic Theory 59, 1-16.

Anderson, E.J. and X. Hu (2008), Forward contracts and market power in electricity market, International Journal of Industrial Organization 26, 679-694.

Anderson, E.J. and A.B. Philpott (2002), Using supply functions for offering generation into an electricity market, Operations Research 477-489.

Barlow, M. T. (2002), A diffusion model for electricity prices, Mathematical Finance 287-298.

Baron, D.P. (1977), On the utility theoretic foundations of mean-variance analysis, The Journal of Finance 32, 1683-1697.

Bessembinder, H. (1991), Forward contracts and firm value: investment incentive and contracting effects, Journal of Financial and quantitative Analysis 26, 519-532.

Bessembinder, H. and M. Lemmon (2002), Equilibrium pricing and optimal hedging in electricity forward markets, Journal of Finance 13471382. 
Birol, F. (2009), World Energy Outlook, Technical report, Technical report, IEA/OECD, Paris, France.

Bolle, F. (1992), Supply function equilibria and the danger of tacit collusion: The case of spot markets for electricity, Energy Economics 14, 94-102.

Boyle, G.W. and G.A. Guthrie (2006), Hedging the value of waiting, Journal of Banking E Finance 30, 1245-1267.

Brennan, T.J. (2003), Electricity capacity requirements: who pays?, The Electricity Journal 16, 11-22.

Bushnell, J. (2007), Oligopoly equilibria in electricity contract markets, Journal of Regulatory Economics 32, 225-245.

Bushnell, J., E. Mansur, and C. Saravia (2008), Vertical arrangements, market structure, and competition: An analysis of restructured US electricity markets, American Economic Review 98, 237-266.

Cartea, A., M.G. Figueroa, and H. Geman (2009), Modeling electricity prices with forward looking capacity constraints, Applied Mathematical Finance 16, 103-122.

Cartea, A. and P. Villaplana (2005), Pricing in electricity markets: a mean reverting jump diffusion model with seasonality, Applied Mathematical Finance 313-335.

(2008), Spot price modeling and the valuation of electricity forward contracts: The role of demand and capacity, Journal of Banking $\mathcal{E}$ Finance 32, 2502-2519, URL http: / / ideas.repec.org/a/ eee/jbfina/v32y2008i12p2502-2519.html.

Cartea, Figueiroa M., A. and H. Geman (2009), Modelling electricity prices with forward looking capacity constraints, Applied Mathematical Finance 16(2), 103-122. 
Cramton, P. and S. Stoft (2007), Why we need to stick with uniform-price auctions in electricity markets, The Electricity Journal 20, 26-37.

Deng, S. (2000), Stochastic models of energy commodity prices and their applications: mean-reversion with jumps and spikes (University of California Energy Institute).

Dixit, A. (1983), Vertical integration in a monopolistically competitive industry, International Journal of Industrial Organization 1, 63-78.

Dixit, A.K., R.S. Pindyck, and G.A. Davis (1994), Investment under uncertainty, volume 15 (Princeton University Press).

Enders, W. (1995), Applied econometric time series (Wiley).

Evans, L. and G Guthrie (2009), How options provided by storage affect electricity prices, Southern Economic Journal 75, 681-702, URL http://ideas.repec.org/a/sej/ancoec/ v753y2009p681-702.html.

Evans, L.T., G.A. Guthrie, and A. Lu (2010), A New Zealand electricity market model: assessment of the effect of climate change on electricity production and consumption.

Falbo, P., D. Felletti, and S. Stefani (2010), Integrated risk management for an electricity producer, European Journal of Operational Research 207, 1620-1627.

Ferreira, J.L. (2003), Strategic interaction between futures and spot markets, Journal of Economic Theory 108, 141-151.

Froot, K.A., D.S. Scharfstein, and J.C. Stein (1993), Risk management: coordinating corporate investment and financing policies, Journal of Finance $48,1629-1658$. 
Gans, J.S., F.A. Wolak, and VIC Carlton (2008), A comparison of ex ante versus ex post vertical market power: evidence from the electricity supply industry, Technical report, Working paper, SSRN.

Geman, H. and A. Roncoroni (2006), Understanding the fine structure of electricity prices, Journal of Business 79, 1225.

Green, R.J. (1999), The electricity contract market in England and Wales, Journal of Industrial Economics 47, 107-124.

Green, R.J. and C. Le Coq (2006), The length of the contract and collusion, CSEM working paper 154.

Green, R.J. and D.M. Newbery (1992), Competition in the British electricity spot market, Journal of political economy 929-953.

Greene, W. and C. Zhang (2003), Econometric analysis, volume 5th Edition (Prentice hall New Jersey).

Grossman, S.J. and O.D. Hart (1986), The costs and benefits of ownership: A theory of vertical and lateral integration, The Journal of Political Economy 94, 691-719.

Hart, O. and O.D. Hart (1995), Firms, contracts, and financial structure (Oxford University Press, USA).

Hart, O. and J. Moore (1990), Property rights and the nature of the firm, The Journal of Political Economy 98, 1119-1158.

Hart, O., J. Tirole, D.W. Carlton, and O.E. Williamson (1990), Vertical integration and market foreclosure, Brookings papers on economic activity. Microeconomics 1990, 205-286.

Harte, D., M. Pickup, and PJ Thomson (2004), Stochastic models for hydro catchment inflows: an exploratory analysis, $A$ report commissioned by the New Zealand Electricity Commission . 
Heaton, J. and D.J. Lucas (1996), Evaluating the effects of incomplete markets on risk sharing and asset pricing, The Journal of Political Economy 104, 443-487.

Hogan, S. (2010), Does wholesale market power extend to fixed-price forward prices in electricity markets? .

Holmberg, P. and D. Newbery (2010), The supply function equilibrium and its policy implications for wholesale electricity auctions, Utilities Policy 18, 209-226.

Hortacsu, A. and S.L. Puller (2005), Understanding strategic bidding in restructured electricity markets: a case study of ERCOT, NBER Working Paper .

(2008), Understanding strategic bidding in multi-unit auctions: a case study of the Texas electricity spot market, The RAND Journal of Economics 39, 86-114.

Hughes, W. R. and A. Parece (2002), The economics of price spikes in deregulated power markets, The Electricity Journal 15, 31-44.

Joskow, P. (2005), Vertical integration, Handbook of New Institutional Economics 319-348.

Kahn, A.E., P.C. Cramton, R.H. Porter, and R.D. Tabors (2001), Uniform pricing or pay-as-bid pricing:: a dilemma for California and beyond, The Electricity Journal 14, 70-79.

Kastl, J. (2006), Discrete bids and empirical inference in divisible good auctions, Mimeo, Department of Economics, Stanford University. .

(2008), On the properties of equilibria in private value divisible good auctions with constrained bidding, Mimeo, Department of Economics, Stanford University. . 
Klein, B. (2000), Fisher-General Motors and the nature of the firm, Journal of Law and Economics 105-141.

Klein, B., R.G. Crawford, and A.A. Alchian (1978), Vertical integration, appropriable rents, and the competitive contracting process, JL E econ. 21, 297.

Klein, B. and K.B. Leffler (1981), The role of market forces in assuring contractual performance, The Journal of Political Economy 89, 615-641.

Klemperer, P.D. and M.A. Meyer (1989), Supply function equilibria in oligopoly under uncertainty, Econometrica 57, 1243-1277.

Levy, H. and H.M. Markowitz (1979), Approximating expected utility by a function of mean and variance, The American Economic Review 69, 308-317.

Liski, M. and J-P. Montero (2006), Forward trading and collusion in oligopoly, Journal of Economic Theory 131, 212-230.

Lucia, J. and E.S. Schwartz (2002), Electricity prices and power derivatives: evidence from the nordic power exchange, Review of Derivatives Research 5-50.

Lyle, M. R. and R. J. Elliott (2009), A simple hybrid model for power derivatives, Energy Economics 31, 757-767.

Mahenc, P. and F. Salanie (2004), Softening competition through forward trading, Journal of Economic Theory 116, 282-293.

Mason, G.M. (2002), A comparison of restructured electricity pools, the key characteristics of NZEM spot prices, and the estimation of a two-factor mean reverting model, Ph.D. thesis.

Meade, R. and S. O'Connor (2009), Comparison of long-term contracts and vertical integration in decentralised electricity markets . 
Meade, Richard and Lewis T. Evans (2006), Alternating currents or counter-revolution? Contemporary electricity reform in $\mathrm{New}$ Zealand, Victoria University Press .

Mello, A.S. and J.E. Parsons (2000), Hedging and liquidity, Review of Financial Studies 13, 127.

Newbery, D.M. (1998), Competition, contracts and entry in the electricity spot market, Rand Journal of Economics 29.

Ordover, J.A., G. Saloner, and S.C. Salop (1990), Equilibrium vertical foreclosure, The American Economic Review 80, 127-142.

Oren, S. (2004), When is a pay-as bid preferable to uniform price in electricity markets, in Power Systems Conference and Exposition, 2004. IEEE PES, 1618-1620 (IEEE).

Perry, M.K. (1978), Price discrimination and forward integration, The Bell Journal of Economics 9, 209-217.

Pirrong, C. and M. Jermakyan (2008), The price of power: The valuation of power and weather derivatives, Journal of Banking and Finance .

Porchet, A., R. Ad, N. Touzi, and G. Chemla (2009), Forward hedging and vertical integration in electricity markets, EFA 2009 Bergen Meetings Paper URL http: / / ssrn. com/abstract $=1342874$.

Pulley, L.B. (1981), A general mean-variance approximation to expected utility for short holding periods, Journal of Financial and Quantitative Analysis 16, 361-373.

Schmalensee, R., M. Armstrong, and R.H. Porter (2007), Handbook of industrial organization (North Holland).

Schwartz, E.S. (1997), The stochastic behavior of commodity prices: implications for valuation and hedging, Journal of Finance 52, 923-974. 
Schwartz, E.S. and J.E. Smith (2000), Short-term variations and long-term dynamics in commodity prices, Management Science 46, 893-911.

Skantze, P., A. Gubina, and M. Ilić (2000), Bid-based stochastic model for electricity prices: the impact of fundamental drivers on market dynamics (Massachusetts Institute of Technology Cambridge, Energy Laboratory).

Stoft, S. (2002), Power system economics: designing markets for electricity, volume 2 (IEEE press).

Stulz, R.M. (1984), Optimal hedging policies, Journal of Financial and Quantitative Analysis 19, 127-140.

- (1990), Managerial discretion and optimal financing policies, Journal of financial Economics 26, 3-27.

Tirole, J. (2000), The theory of industrial organization (MIT press).

UMR (2008), Electricity hedge market issues: a qualitative and quantitative study, Technical report, Electricity Commission, New Zealand.

Von der Fehr, N.-H. M. and D. Harbord (1993), Spot market competition in the UK electricity industry, Economic Journal 103, 531-546.

Willems, B. and J. Morbee (2010), Market completeness: How options affect hedging and investments in the electricity sector, Energy Economics 32, 786-795.

Willems, Bert and Joris Morbee (2008), Risk management in electricity markets: hedging and market incompleteness, SSRN eLibrary .

Williamson, O.E. (1971), The vertical integration of production: market failure considerations, The American Economic Review 61, 112-123.

- (1983), Credible commitments: Using hostages to support exchange, The American Economic Review 73, 519-540. 
(1985), The economic institutions of capitalism: Firms, Markets, Relational Contracting, New York.

(2000), The new institutional economics: taking stock, looking ahead, Journal of economic literature 38, 595-613.

Wilson, R. (2008), Supply function equilibrium in a constrained transmission system, Operations Research 56, 369.

Wilson, Robert (1979), Auctions of shares, The Quarterly Journal of Economics 93, 675-89, URL http://ideas.repec.org/a/tpr/ qjecon/v93y1979i4p675-89.html.

Wolak, F.A. (2000), An empirical analysis of the impact of hedge contracts on bidding behavior in a competitive electricity market, International Economic Journal 14, 1-39.

(2003), Measuring unilateral market power in wholesale electricity markets: the California market, 1998-2000, The American economic review 93, 425-430. 


\section{Appendix A}

\section{Chapter 2}

\section{A.1 Proof of lemma 2.2.1}

To solve this maximization problem we need first to integrate by parts the objective function. Suppressing the $i$ and $t$ indices we have, modulo a constant term:

$$
\begin{array}{cl}
-\int_{\underline{p}}^{\bar{p}} \quad U^{\prime}(S(p) p-C(S(p), \vec{W})-(p-P C) Q C)\left(S^{\prime}(p) p-\right. \\
\left.\quad C^{\prime}(S(p), \vec{W}) S^{\prime}(p)-Q C\right) H(p, S(p) ; Q C) d p
\end{array}
$$

Labeling the integrand:

$$
\begin{aligned}
F\left(p, S, S^{\prime}\right)=\quad & -U^{\prime}(S(p) p-C(S(p), \vec{W})-(p-P C) Q C)\left(S^{\prime}(p) p\right. \\
& \left.+S(p)-C^{\prime}(S(p), \vec{W}) S^{\prime}(p)-Q C\right) H(p, S(p) ; Q C),
\end{aligned}
$$

from the calculus of variation, the Euler-Lagrange necessary condition for the optimal $S(p)$ is given by:

$$
\frac{d}{d p} F_{S^{\prime}}=F_{S} .
$$


Evaluating the derivatives:

$$
\begin{aligned}
-F_{S}= & H_{S} U^{\prime}(.)\left[p S^{\prime}+S-C^{\prime}(S, \vec{W}) S^{\prime}-Q C\right] \\
& +H U^{\prime \prime}(.)\left(p-C^{\prime}(S, \vec{W})\right)\left[p S^{\prime}+S-C^{\prime}(S, \vec{W}) S^{\prime}-Q C\right] \\
& +H U^{\prime}(.)\left[1-C^{\prime \prime}(S, \vec{W}) S^{\prime}\right] \\
-F_{S^{\prime}}= & H U^{\prime}(.)\left[p-C^{\prime}(S, \vec{W})\right],
\end{aligned}
$$

and taking the total derivative of $F_{S^{\prime}}$ with respect to $p$ :

$$
\begin{aligned}
-\frac{d}{d p} F_{S^{\prime}}= & H_{p} U^{\prime}(.)\left[p-C^{\prime}(S, \vec{W})\right]+H_{S} S^{\prime} U^{\prime}(.)\left[p-C^{\prime}(S, \vec{W})\right] \\
& +H U^{\prime \prime}(.)\left[p S^{\prime}+S-C^{\prime}(S, \vec{W}) S^{\prime}-Q C\right]\left(p-C^{\prime}(S, \vec{W})\right) \\
& +H U^{\prime}(.)\left(1-C^{\prime \prime}(S, \vec{W}) S^{\prime}\right) .
\end{aligned}
$$

Equating and canceling terms we get:

$$
H_{S} U^{\prime}(.)(S-Q C)=H_{p} U^{\prime}(.)\left(p-C^{\prime}(S, \vec{W})\right)
$$

Considering again the $i$ and $t$ indices we have:

$$
p-M C_{i t}\left(S_{i t}^{*}(p), \vec{W}_{t}\right)=\left[S_{i t}^{*}(p)-Q C_{i t}\right] \frac{H_{S}\left(p, S_{i t}^{*}(p) ; Q C_{i t}\right)}{H_{p}\left(p, S_{i t}^{*}(p) ; Q C_{i t}\right)} .
$$

\section{A.2 Proof of lemma 2.2.2}

Suppose that,

$$
S_{i}\left(p, Q C_{i}, \vec{W}\right)=\alpha_{i}(p)+\beta_{i}\left(Q C_{i}\right)+\sum_{l=1}^{L} \delta_{l i}\left(w_{l}\right)
$$

Given that we can use the spot market clearing condition $\sum_{i=1}^{K} S_{i}=D$ to represent the probability $\operatorname{Pr}\left(p_{t}^{c} \leq p \mid Q C_{i}, S_{i}(p)\right)$ : 


$$
\sum_{j \neq i} \beta_{j}\left(Q C_{j}\right)+\sum_{j \neq i} \sum_{k=1}^{M} \delta_{k j}\left(w_{k}\right)-\varepsilon \geq D\left(p^{R}, \vec{W}_{t}\right)-S_{i}-\sum_{j \neq i} \alpha_{j}(p) .
$$

The left hand side of this inequality can be labeled as a (bidder specific) random variable, $\theta_{i}$ that does not depend on $p$, and the right hand side is a deterministic function of price. Let $\Gamma_{i}($.$) denote the cdf of \theta_{i}$ and $\gamma_{i}($. denote the pdf (both conditional on the bidder's contract quantity $Q C_{i}$. Given these:

$$
\begin{aligned}
H_{p}\left(p, S_{i} ; Q C_{i}\right)= & \frac{\partial}{\partial p} \operatorname{Pr}\left(p_{t}^{c} \leq p \mid Q C_{i}, S_{i}\right) \\
= & \frac{\partial}{\partial p} \operatorname{Pr}\left(\theta_{i} \geq D\left(p^{R}, \vec{W}_{t}\right)-S_{i}-\sum_{j \neq i} \alpha_{j}(p)\right) \\
= & \frac{\partial}{\partial p}\left[1-\Gamma_{i}\left(D\left(p^{R}, \vec{W}_{t}\right)-S_{i}-\sum_{j \neq i} \alpha_{j}(p)\right)\right] \\
= & -\gamma_{i}\left(D\left(p^{R}, \vec{W}_{t}\right)-S_{i}-\sum_{j \neq i} \alpha_{j}(p)\right) \frac{\partial}{\partial p}\left(D\left(p^{R}, \vec{W}_{t}\right)\right. \\
& \left.-S_{i}-\sum_{j \neq i} \alpha_{j}(p)\right),
\end{aligned}
$$

and

$$
\begin{aligned}
H_{S}\left(p, S_{i} ; Q C_{i}\right)= & \frac{\partial}{\partial S_{i}} \operatorname{Pr}\left(p_{t}^{c} \leq p \mid Q C_{i}, S_{i}\right) \\
= & -\gamma_{i}\left(D\left(p^{R}, \vec{W}_{t}\right)-S_{i}-\sum_{j \neq i} \alpha_{j}(p)\right) \frac{\partial}{\partial S_{i}}\left(D\left(p^{R}, \vec{W}_{t}\right)\right. \\
& \left.-S_{i}-\sum_{j \neq i} \alpha_{j}(p)\right) .
\end{aligned}
$$

Evaluating the derivatives gives $\frac{H_{p}\left(p, S_{i} ; Q C_{i}\right)}{H_{S}\left(p, S_{i} ; Q C_{i}\right)}=-\left[D^{\prime}\left(p^{R}, \vec{W}_{t}\right)-\sum_{j \neq i} \alpha_{j}^{\prime}(p)\right]$, which is the derivative in respect to price of the residual demand faced by firm $i$. 
Substituting these results in the equation (2.4), defining the marginal cost $C_{i}^{\prime}\left(S_{i}(p), \vec{W}\right)$ as $M C_{i}\left(S_{i}, \vec{W}\right)$ and considering again the index $\mathbf{t}$, we yield the following equation:

$$
p_{t}-M C_{i t}\left(S_{i t}, \vec{W}_{t}\right)=\frac{S_{i t}-Q C_{i t}}{\frac{\partial \sum_{j \neq i} S_{j t}}{\partial p}} .
$$

Now let us consider $q_{i t}=\tilde{D}_{t}-\sum_{j \neq i} S_{j t}-Q C_{i t}$. The elasticity $\varepsilon_{i t}$ is equal to $\frac{d q_{i t}}{d p_{t}} \frac{p_{t}}{q_{i t}}$. Observe that $\frac{d q_{i t}}{d p_{t}}=-\frac{\partial \sum_{j \neq i} S_{j t}}{\partial p_{t}}$ and, in equilibrium, $D_{t}-$ $\sum_{j \neq i} S_{j t}=S_{i t}$. Therefore $q_{i t}=S_{i t}-Q C_{i t}$ and,

$$
\frac{p_{t}-M C_{i t}}{p_{t}}=-\frac{1}{\varepsilon_{i t}\left(q_{i t}\right)} \text {. }
$$

\section{A.3 Proof of proposition 2.2.3}

The assumed general form of the symmetric Bayesian-Nash equilibria is a linear supply function of the form:

$$
S_{i t}\left(p_{t}, Q C_{i t}, \vec{W}_{t}\right)=\psi+\alpha p_{t}+\beta Q C_{i t}+\sum_{j=1}^{L} \delta_{j} w_{j t} .
$$

Now substituting and suppressing time subscript $t$, we have:

$$
\begin{aligned}
& p-a-\sum_{j=1}^{L} \rho_{j} w_{j}-b S_{i}=\frac{Q C_{i}-S_{i}}{-(K-1) \alpha} \\
& (K-1) \alpha p-(K-1) \alpha a-(K-1) \alpha \sum_{j=1}^{L} \rho_{j} w_{j t}+Q C_{i} \\
& =S_{i}(p)[1+(K-1) \alpha b] .
\end{aligned}
$$


Reorganizing equations we have:

$$
\begin{aligned}
& (K-1) \alpha p-(K-1) \alpha a-(K-1) \alpha \sum_{j=1}^{L} \rho_{j} w_{j t}+Q C_{i} \\
& =\left(\psi+\alpha p+\beta Q C_{i}+\sum_{j=1}^{L} \delta_{j} w_{j}\right)[1+(K-1) \alpha b]
\end{aligned}
$$

which implies that the following must hold for $i=1,2, \ldots N$ :

$$
\begin{aligned}
& \frac{(K-1) \alpha}{1+(K-1) \alpha b}=\alpha \\
& -\frac{a(K-1) \alpha}{1+(K-1) \alpha b}=\psi \\
& \frac{1}{1+(K-1) \alpha b}=\beta \\
& -\frac{\rho_{1}(K-1) \alpha}{1+(K-1) \alpha b}=\delta_{1} \\
& -\frac{\rho_{2}(K-1) \alpha}{1+(K-1) \alpha b}=\delta_{2} \\
& \vdots \\
& -\frac{\rho_{L}(K-1) \alpha}{1+(K-1) \alpha b}=\delta_{L} .
\end{aligned}
$$

This system of equations has 2 solutions. In the first $\alpha=\psi=\delta_{j}=0$ and $\beta=1$. However, this solution corresponds to a vertical supply curve which would not in general clear the market for our inelastic demand. The second solution is given by $\alpha=\frac{K-2}{b(K-1)}, \psi=-\frac{a(K-2)}{b(K-1)}, \delta_{j}=-\frac{\rho_{j}(K-2)}{b(K-1)}$ and $\beta=\frac{1}{K-1}$. Substituting this solution back into the assumed general supply function,

$$
S_{i t}\left(p_{t}, Q C_{i t}, \vec{W}_{t}\right)=-\frac{a(K-2)}{b(K-1)}+\frac{K-2}{b(K-1)} p_{t}+\frac{1}{K-1} Q C_{i t}+
$$




$$
\begin{gathered}
\frac{-(K-2)}{b(K-1)} \sum_{j=1}^{L} \rho_{j} w_{j t} \\
\tilde{D}_{t}\left(p_{t}^{R}, \vec{W}_{t}\right)=c-\kappa_{0} p^{R}+\sum_{j=1}^{L} \kappa_{j} w_{j t}+\epsilon_{t} .
\end{gathered}
$$

Putting in terms of $p$,

$$
\begin{aligned}
p_{t}= & a+\frac{b(K-1)\left(c-\kappa_{0} p^{R}\right)}{K(K-2)}+\sum_{j=1}^{L}\left(\frac{b(K-1)}{K(K-2)} \kappa_{j}+\rho_{j}\right) w_{j t}-\frac{b}{K(K-2)} Q C_{i t} \\
& -\frac{b}{K(K-2)} \sum_{j \neq i} Q C_{j t}+\frac{b(K-1)}{K(K-2)} \epsilon_{t} \\
= & A-B \sum_{i=1}^{N} Q C_{i t}+\sum_{j=1}^{L} C_{j} w_{j t}+(K-1) B \epsilon_{t} .
\end{aligned}
$$




\section{Appendix B}

\section{Chapter 3}

\section{B.1 Proof of lemma 3.3.1}

To solve this maximization problem we need first to integrate by parts the objective function. Suppressing the $i$ and $t$ indices we have, modulo a constant term:

$$
\begin{array}{cl}
-\int_{\underline{p}}^{\bar{p}} \quad U^{\prime}\left(S(p) p-C(S(p), \vec{W})-(p-P C) Q C-m_{i}\left(p-p^{R}\right) D\left(p_{t}^{R}, \vec{W}_{t}\right)\right) \\
\quad \times\left(S^{\prime}(p) p-C^{\prime}(S(p), \vec{W}) S^{\prime}(p)-Q C\right) H(p, S(p) ; Q C) d p .
\end{array}
$$

Labeling the integrand:

$$
\begin{aligned}
F= & -U^{\prime}\left(S(p) p-C(S(p), \vec{W})-(p-P C) Q C-m\left(p-p^{R}\right) D\left(p_{t}^{R}, \vec{W}_{t}\right)\right) \\
& \times\left(S^{\prime}(p) p+S(p)-C^{\prime}(S(p), \vec{W}) S^{\prime}(p)-Q C-m D\left(p_{t}^{R}, \vec{W}_{t}\right)\right) \\
& \times H(p, S(p) ; Q C) .
\end{aligned}
$$

From the calculus of variation, the Euler-Lagrange necessary condition for the optimal $S(p)$ is given by:

$$
\frac{d}{d p} F_{S^{\prime}}=F_{S}
$$


Evaluating the derivatives:

$$
\begin{aligned}
-F_{S}= & \left.H_{S} U^{\prime}(.)\left[p S^{\prime}+S-C^{\prime}(S, \vec{W}) S^{\prime}-Q C-m D\left(p_{t}^{R}, \vec{W}_{t}\right)\right)\right] \\
& \left.+H U^{\prime \prime}(.)\left(p-C^{\prime}(S, \vec{W})\right)\left[p S^{\prime}+S-C^{\prime}(S, \vec{W}) S^{\prime}-Q C-m D\left(p_{t}^{R}, \vec{W}_{t}\right)\right)\right] \\
& +H U^{\prime}(.)\left[1-C^{\prime \prime}(S, \vec{W}) S^{\prime}\right] \\
-F_{S^{\prime}}= & H U^{\prime}(.)\left[p-C^{\prime}(S, \vec{W})\right] .
\end{aligned}
$$

And taking the total derivative of $F_{S^{\prime}}$ with respect to $p$ :

$$
\begin{aligned}
-\frac{d}{d p} F_{S^{\prime}}= & H_{p} U^{\prime}(.)\left[p-C^{\prime}(S, \vec{W})\right]+H_{S} S^{\prime} U^{\prime}(.)\left[p-C^{\prime}(S, \vec{W})\right] \\
& \left.+H U^{\prime \prime}(.)\left[p S^{\prime}+S-C^{\prime}(S, \vec{W}) S^{\prime}-Q C-m D\left(p_{t}^{R}, \vec{W}_{t}\right)\right)\right]\left(p-C^{\prime}(S, \vec{W})\right) \\
& +H U^{\prime}(.)\left(1-C^{\prime \prime}(S, \vec{W}) S^{\prime}\right) .
\end{aligned}
$$

Equating and canceling terms we get:

$$
\left.H_{S} U^{\prime}(.)\left(S-Q C-m D\left(p_{t}^{R}, \vec{W}_{t}\right)\right)\right)=H_{p} U^{\prime}(.)\left(p-C^{\prime}(S, \vec{W})\right) .
$$

Considering again the $i$ and $t$ indices we have:

$$
\left.p-M C_{i t}\left(S_{i t}^{*}(p), \vec{W}_{t}\right)=\left[S_{i t}^{*}(p)-Q C_{i t}-m_{i} D\left(p_{t}^{R}, \vec{W}_{t}\right)\right)\right] \frac{H_{S}\left(p, S_{i t}^{*}(p) ; Q C_{i t}\right)}{H_{p}\left(p, S_{i t}^{*}(p) ; Q C_{i t}\right)} .
$$

\section{B.2 Proof of lemma 3.3.2}

Suppose that,

$$
S_{i}\left(p, Q C_{i}, \vec{W}\right)=\alpha_{i}(p)+\beta_{i}\left(Q C_{i}\right)+\sum_{j=1}^{L} \delta_{k i}\left(w_{k}\right)
$$


Given that we can use the market clearing condition to represent the event $\operatorname{Pr}\left(p_{t}^{c} \leq p \mid Q C_{i}, S_{i}(p)\right)$ :

$$
\sum_{j \neq i} \beta_{j}\left(Q C_{j}\right)+\sum_{j \neq i} \sum_{k=1}^{M} \delta_{k j}\left(w_{k}\right) \geq D\left(p^{R}, \vec{W}_{t}\right)-S_{i}-\sum_{j \neq i} \alpha_{j}(p) .
$$

The left hand side of this inequality can be labeled as a (bidder specific) random variable, $\theta_{i}$ that does not depend on $p$, and the right hand side is a deterministic function of price. Let $\Gamma_{i}($.$) denote the cdf of \theta_{i}$ and $\gamma_{i}($. denote the pdf (both conditional on the bidder's contract quantity $Q C_{i}$ ). Given these:

$$
\begin{aligned}
H_{p}\left(p, S_{i} ; Q C_{i}\right)= & \frac{\partial}{\partial p} \operatorname{Pr}\left(p_{t}^{c} \leq p \mid Q C_{i}, S_{i}\right) \\
= & \frac{\partial}{\partial p} \operatorname{Pr}\left(\theta_{i} \geq D\left(p^{R}, \vec{W}_{t}\right)-S_{i}-\sum_{j \neq i} \alpha_{j}(p)\right) \\
= & \frac{\partial}{\partial p}\left[1-\Gamma_{i}\left(D\left(p^{R}, \vec{W}_{t}\right)-S_{i}-\sum_{j \neq i} \alpha_{j}(p)\right)\right] \\
= & -\gamma_{i}\left(D\left(p^{R}, \vec{W}_{t}\right)-S_{i}-\sum_{j \neq i} \alpha_{j}(p)\right) \frac{\partial}{\partial p}\left(D\left(p^{R}, \vec{W}_{t}\right)\right. \\
& \left.-S_{i}-\sum_{j \neq i} \alpha_{j}(p)\right),
\end{aligned}
$$

and

$$
\begin{aligned}
H_{S}\left(p, S_{i} ; Q C_{i}\right)= & \frac{\partial}{\partial S_{i}} \operatorname{Pr}\left(p_{t}^{c} \leq p \mid Q C_{i}, S_{i}\right) \\
= & -\gamma_{i}\left(D\left(p^{R}, \vec{W}_{t}\right)-S_{i}-\sum_{j \neq i} \alpha_{j}(p)\right) \frac{\partial}{\partial S_{i}}\left(D\left(p^{R}, \vec{W}_{t}\right)\right. \\
& \left.-S_{i}-\sum_{j \neq i} \alpha_{j}(p)\right) .
\end{aligned}
$$

Evaluating the derivatives gives $\frac{H_{p}\left(p, S_{i} ; Q C_{i}\right)}{H_{S}\left(p, S_{i} ; Q C_{i}\right)}=-\left[\frac{\partial D\left(p^{R}, \vec{W}_{t}\right)}{\partial p}-\sum_{j \neq i} \alpha_{j}^{\prime}(p)\right]$ 
which is the derivative in respect to price of the residual demand faced by firm $i$. By definition of the aggregate demand, $\frac{\partial D\left(p^{R}, \vec{W}_{t}\right)}{\partial p}=0$.

Substituting these results in the equation (3.4), defining the marginal cost $C_{i}^{\prime}\left(S_{i}(p), \vec{W}\right)$ as $M C_{i}\left(S_{i}, \vec{W}\right)$ and considering again the index $t$, we yield the following equation:

$$
\begin{aligned}
p_{t}-M C_{i t} & =-\frac{S_{i t}-Q C_{i t}-m_{i} D_{t}}{\frac{\partial D_{t}}{\partial p_{t}}-\frac{\partial \sum_{j \neq i} S_{j t}}{\partial p_{t}}} \\
p_{t}-M C_{i t} & =\frac{S_{i t}-Q C_{i t}-m_{i} D_{t}}{\frac{\partial \sum_{j \neq i} S_{j t}}{\partial p_{t}}} .
\end{aligned}
$$

Now let's consider $q_{i t}^{\prime}=D_{t}-\sum_{j \neq i} S_{j t}-Q C_{i t}-m_{i} D_{t}$. The elasticity $\varepsilon_{i t}^{\prime}$ is equal to $-\frac{d q_{i t}^{\prime}}{d p_{t}} \frac{p_{t}}{q_{i t}^{\prime}}$. Observe that $\frac{d q_{i t}^{\prime}}{d p_{t}}=-\frac{\partial \sum_{j \neq i} S_{j t}}{\partial p_{t}}$ and, in equilibrium, $D_{t}-\sum_{j \neq i} S_{j t}=S_{i t}$. Therefore $q_{i t}^{\prime}=S_{i t}-Q C_{i t}-m_{i} D_{t}$ and,

$$
\frac{p_{t}-M C_{i t}}{p_{t}}=\frac{1}{\varepsilon_{i t}^{\prime}\left(q_{i t}^{\prime}\right)}
$$

\section{B.3 Proof of proposition 3.3.3}

Let's now assume that the general form of the symmetric Bayesian-Nash equilibria is a linear supply function of the form:

$$
S_{i t}\left(p_{t}, Q C_{i t}, \vec{W}_{t}\right)=\psi_{i}+\alpha p_{t}+\beta Q C_{i t}+\sum_{j=1}^{L} \delta_{j} w_{j t}
$$

Now substituting and suppressing time subscript $t$, we have:

$$
\begin{aligned}
& p-a-\sum_{j=1}^{L} \rho_{j} w_{j}-b S_{i}=\frac{Q C_{i}+m_{i} D-S_{i}}{-(K-1) \alpha} \\
& (K-1) \alpha p-(K-1) \alpha a-(K-1) \alpha \sum_{j=1}^{L} \rho_{j} w_{j t}+Q C_{i}+m_{i}\left(c-\kappa_{o} p^{R}+\sum_{j=1}^{L} \kappa_{j} w_{j t}\right)
\end{aligned}
$$




$$
=S_{i}(p)[1+(K-1) \alpha b] .
$$

Reorganizing equations we have:

$$
\begin{aligned}
& m_{i}\left(c-\kappa_{o} p^{R}\right)-(K-1) \alpha a+(K-1) \alpha p-\sum_{j=1}^{L}\left((K-1) \alpha \rho_{j}-m_{i} \kappa_{j}\right) w_{j t}+Q C_{i} \\
& =\left(\psi_{i}+\alpha p+\beta Q C_{i}+\sum_{j=1}^{L} \delta_{i j} w_{j}\right)[1+(K-1) \alpha b],
\end{aligned}
$$

which implies that the following must hold for $i=1,2, \ldots N$ :

$$
\begin{aligned}
& \frac{(K-1) \alpha}{1+(K-1) \alpha b}=\alpha \\
& \frac{+m_{i}\left(c-\kappa_{o} p^{R}\right)-a(K-1) \alpha}{1+(K-1) \alpha b}=\psi_{i} \\
& \frac{1}{1+(K-1) \alpha b}=\beta \\
& -\frac{\rho_{1}(K-1) \alpha-m_{i} \kappa_{1}}{1+(K-1) \alpha b}=\delta_{i 1} \\
& -\frac{\rho_{2}(K-1) \alpha-m_{i} \kappa_{2}}{1+(K-1) \alpha b}=\delta_{i 2} \\
& \vdots \quad \\
& -\frac{\rho_{L}(K-1) \alpha-m_{i} \kappa_{L}}{1+(K-1) \alpha b}=\delta_{i L}
\end{aligned}
$$

Solving the system and substituting back into the assumed general supply function,

$$
\begin{aligned}
S_{i t}\left(p_{t}, Q C_{i t}, \vec{W}_{t}\right)= & -\frac{a(K-2)}{b(K-1)}+\frac{K-2}{b(K-1)} p_{t}+\frac{1}{K-1} Q C_{i t} \\
& +\frac{m_{i}}{K-1} D_{t}\left(p_{t}^{R}, \vec{W}_{t}\right)-\frac{(K-2)}{b(K-1)} \sum_{j=1}^{L} \rho_{j} w_{j t}
\end{aligned}
$$




$$
D_{t}\left(p_{t}^{R}, \vec{W}_{t}\right)=c-\kappa_{o} p^{R}+\sum_{j=1}^{L} \kappa_{j} w_{j t} .
$$

Clearing the market and putting in terms of $p_{t}^{c}$,

$$
\begin{aligned}
p_{t}^{c}= & a+b \frac{\left(c-\kappa_{o} p^{R}\right)\left(K-\left(1+\sum_{i=1}^{K} m_{i}\right)\right)}{K(K-2)}-\frac{b}{K(K-2)} \sum_{i=1}^{K} Q C_{i t} \\
& +\sum_{j=1}^{L}\left(\rho_{j}+b \frac{\left(K-\left(1+\sum_{i=1}^{K} m_{i}\right)\right)}{K(K-2)} \kappa_{j}\right) w_{j t},
\end{aligned}
$$

which is the same as:

$$
p_{t}^{c}=A-B \sum_{i=1}^{K} Q C_{i t}^{*}+\sum_{j=1}^{L} C_{j} w_{j t},
$$

where

$$
\begin{aligned}
A & =a+b \frac{\left(c-\kappa_{o} p^{R}\right)\left(K-\left(1+\sum_{i=1}^{K} m_{i}\right)\right)}{K(K-2)} \\
B & =\frac{b}{K(K-2)} \\
C_{j} & =\rho_{j}+b \frac{\left(K-\left(1+\sum_{i=1}^{K} m_{i}\right)\right)}{K(K-2)} \kappa_{j} .
\end{aligned}
$$

\section{B.4 Proof of proposition 3.4.1}

Assume that $H_{i t}\left(p, \hat{S}_{i t}(p) ; Q C_{k t}^{*}\right) \equiv \operatorname{Pr}\left(p_{t}^{c} \leq p \mid \hat{S}_{i t}(p), Q C_{k t}^{*}\right) \forall k=1,2 \ldots N$. Therefore, by the market clearing condition $\left(\varepsilon=S_{i t}\left(p_{t}^{c}, \vec{W}_{t}\right)+\sum_{j \neq i} S_{j}\left(p_{t}^{c}, \vec{W}\right)-\right.$ $\left.D\left(p^{R}, \vec{W}_{t}\right)\right)$, we know that $\epsilon_{t}$ is a function $\epsilon_{t}\left(p_{t}^{c}, S_{i t}\left(p_{t}^{c}\right)\right)$ of the spot market clearing price $p_{t}^{c}$ and the firm $i^{\prime}$ s bid $S_{i t}$. 
A vertically integrated firm has the following maximization problem:

$$
\begin{array}{rr}
\max _{\hat{S}_{i t}(p)} \int_{\underline{p}}^{\bar{p}} \quad U_{i}\left[\hat{S}_{i t}(p) p-C_{i t}\left(\hat{S}_{i t}(p), \vec{W}_{t}\right)+m_{i}\left(p_{t}^{R}-p_{t}^{c}\right)\left(D_{t}\left(p_{t}^{R}, \vec{W}_{t}\right)\right.\right. \\
\left.\left.+\epsilon_{t}\left(p, \hat{S}_{i t}(p)\right)\right)+\left(P C_{i t}-p\right) Q C_{i t}\right] d H_{i t}\left(p, \hat{S}_{i t}(p) ; Q C_{i t}\right) .
\end{array}
$$

To solve this maximization problem, we integrate by parts the price integral of the objective function. Suppressing the $i$ and $t$ indices we have, modulo a constant term:

$$
\int_{\underline{p}}^{\bar{p}} F\left(p, S, S^{\prime}\right) d p .
$$

Labeling the integrand:

$$
\begin{aligned}
F\left(p, S, S^{\prime}\right)= & -\left[S^{\prime}(p) p+S(p)-C^{\prime}(S(p), \vec{W}) S^{\prime}(p)-Q C-m\left(D\left(p^{R}, \vec{W}\right)\right.\right. \\
& \left.+\varepsilon(p, S(p))-m p\left(\frac{\partial \varepsilon(p, S(p))}{\partial p}+\frac{\partial \varepsilon(p, S(p))}{\partial S} S^{\prime}(p)\right)\right] \\
& \times H(p, S(p) ; Q C),
\end{aligned}
$$

from the calculus of variation, the Euler-Lagrange necessary condition for the optimal $S(p)$ is given by:

$$
\frac{d}{d p} F_{S^{\prime}}=F_{S}
$$

Evaluating the derivatives:

$$
\begin{aligned}
-F_{S}= & H_{S}\left[p S^{\prime}+S-C^{\prime}(S, \vec{W}) S^{\prime}-Q C-m\left(D\left(p^{R}, \vec{W}\right)+\varepsilon(p, S)\right.\right. \\
& \left.-m p\left(\frac{\partial \varepsilon(p, S)}{\partial p}+\frac{\partial \varepsilon(p, S)}{\partial S} S^{\prime}\right)\right]+H\left[1-C^{\prime \prime}(S, \vec{W}) S^{\prime}\right. \\
& \left.-m \frac{\partial \varepsilon(p, S)}{\partial S}-m p\left(\frac{\partial^{2} \varepsilon(p, S)}{\partial p \partial S}+\frac{\partial^{2} \varepsilon(p, S)}{\partial S^{2}} S^{\prime}\right)\right] \\
-F_{S^{\prime}}= & H\left[p-C^{\prime}(S, \vec{W})-m p \frac{\partial \varepsilon(p, S)}{\partial S}\right],
\end{aligned}
$$


and taking the total derivative of $F_{S^{\prime}}$ with respect to $p$ :

$$
\begin{aligned}
-\frac{d}{d p} F_{S^{\prime}}= & H_{p}\left[p-C^{\prime}(S, \vec{W})-m p \frac{\partial \varepsilon(p, S)}{\partial S}\right] \\
& +H_{S} S^{\prime}\left[p-C^{\prime}(S, \vec{W})-m p \frac{\partial \varepsilon(p, S(p))}{\partial S}\right] \\
& +H\left[1-C^{\prime \prime}(S, \vec{W}) S^{\prime}-m \frac{\partial \varepsilon(p, S)}{\partial S}\right. \\
& \left.-m p\left(\frac{\partial^{2} \varepsilon(p, S)}{\partial p \partial S}+\frac{\partial^{2} \varepsilon(p, S)}{\partial S^{2}} S^{\prime}\right)\right] .
\end{aligned}
$$

Equating and canceling terms we get:

$$
\begin{aligned}
& H_{S}\left[S-Q C-m\left(D\left(p^{R}, \vec{W}\right)+\varepsilon(p, S)\right)-m p \frac{\partial \varepsilon(p, S)}{\partial p}\right] \\
= & H_{p}\left[p-C^{\prime}(S, \vec{W})-m p \frac{\partial \varepsilon(p, S)}{\partial S}\right] .
\end{aligned}
$$

We know by the market clearing condition that:

$$
\begin{aligned}
\varepsilon\left(p, S_{i}\right) & =S_{i}+\sum_{j \neq i} S_{j}\left(p, Q C_{j}, \vec{W}\right)-D\left(p^{R}, \vec{W}\right) \\
\frac{\partial \varepsilon\left(p, S_{i}\right)}{\partial S_{i}} & =1 \\
\frac{\partial \epsilon\left(p, S_{i}\right)}{\partial p} & =\sum_{j \neq i} S_{j}^{\prime} .
\end{aligned}
$$

Thus, we have the following first order condition:

$$
\begin{aligned}
p-C_{i}^{\prime}= & {\left[S_{i}-Q C_{i}-m_{i} S\right] \frac{H_{S}}{H_{p}} } \\
& +m_{i} p\left(1-\sum_{j \neq i} S_{j}^{*^{\prime}} \frac{H_{S}}{H_{p}}\right) .
\end{aligned}
$$


Now suppose that the supply is the following,

$$
S_{i}\left(p, Q C_{i}, W\right)=\psi_{i}+\alpha_{i} p+\beta_{i} Q C_{i}+\sum_{k=1}^{L} \delta_{k i} w_{k}
$$

Given that we can use the market clearing condition to represent the event $\operatorname{Pr}\left(p_{t}^{c} \leq p \mid Q C_{i}, Q C_{j}, S_{i}(p)\right)$ :

$$
\sum_{j \neq i} \psi_{j}+\sum_{j \neq i} \beta_{j} Q C_{j}+\sum_{j \neq i} \sum_{k=1}^{L} \delta_{k j} w_{k}-\varepsilon \geq D\left(p^{R}, W\right)-S_{i}-\sum_{j \neq i} \alpha_{j} p
$$

The left hand side of this inequality can be labeled as a (bidder specific) random variable, $\theta_{i}$ that does not depend on $p$, and the right hand side is a deterministic function of price. Let $\Gamma_{i}($.$) denote the cdf of \theta_{i}$ and $\gamma_{i}($. denote the pdf.

Given that:

$$
\begin{aligned}
H_{p}\left(p, S_{i}\right)= & \frac{\partial}{\partial p} \operatorname{Pr}\left(p_{t}^{c} \leq p \mid S_{i}\right) \\
= & \frac{\partial}{\partial p} \operatorname{Pr}\left(\theta_{i} \geq D\left(p^{R}, W\right)-S_{i}-\sum_{j \neq i} \alpha_{j} p\right) \\
= & \frac{\partial}{\partial p}\left[1-\Gamma_{i}\left(D\left(p^{R}, W\right)-S_{i}-\sum_{j \neq i} \alpha_{j} p\right)\right] \\
= & -\gamma_{i}\left(D\left(p^{R}, W\right)-S_{i}-\sum_{j \neq i} \alpha_{j} p\right) \frac{\partial}{\partial p}\left(D\left(p^{R}, W\right)\right. \\
& \left.-S_{i}-\sum_{j \neq i} \alpha_{j} p\right)
\end{aligned}
$$

and

$$
H_{S}\left(p, S_{i}\right)=\frac{\partial}{\partial S_{i}} \operatorname{Pr}\left(p_{t}^{c} \leq p \mid S_{i}\right)
$$




$$
\begin{aligned}
= & -\gamma_{i}\left(D\left(p^{R}, W\right)-S_{i}-\sum_{j \neq i} \alpha_{j} p\right) \frac{\partial}{\partial S_{i}}\left(D\left(p^{R}, W\right)\right. \\
& \left.-S_{i}-\sum_{j \neq i} \alpha_{j} p\right),
\end{aligned}
$$

evaluating the derivatives gives $\frac{H_{p}\left(p, S_{i}\right)}{H_{S}\left(p, S_{i}\right)}=-\left[\frac{\partial D\left(p^{R}, W\right)}{\partial p}-\sum_{j \neq i} \alpha_{j}\right]=\sum_{j \neq i} \frac{\partial S_{j t}}{\partial p_{t}}$, which is the derivative with respect to price of the residual demand faced by firm $i$. By definition of the aggregate demand, $\frac{\partial D\left(p^{r}, W\right)}{\partial p}=0$.

Therefore, substituting these results in (B.1) we have:

$$
\begin{aligned}
p_{t}-M C_{i t}= & \frac{S_{i t}^{*}-Q C_{i t}-m_{i} S_{t}}{\sum_{j \neq i} \frac{\partial S_{j t}}{\partial p_{t}}} \\
& +m_{i} p\left(1-\frac{\sum_{j \neq i} \frac{\partial S_{j t}}{\partial p_{t}}}{\sum_{j \neq i} \frac{\partial S_{j t}}{\partial p_{t}}}\right) \\
= & \frac{S_{i t}^{*}-Q C_{i t}-m_{i} S_{t}}{\sum_{j \neq i} \frac{\partial S_{j t}}{\partial p_{t}}} .
\end{aligned}
$$

\section{B.5 Proof of proposition 3.4.2}

Assume that supply is $S_{i}\left(p, Q C_{i}, W\right)=\psi_{i}+\alpha_{i} p+\beta_{i} Q C_{i}+\sum_{l=1}^{L} \delta_{i l} w_{l}$. Skipping the subscript $t$ and applying equation B.2, we have the following:

$$
\begin{aligned}
& \sum_{j \neq i} \alpha_{j} p-\sum_{j \neq i} \alpha_{j} a-\sum_{j \neq i} \alpha_{j} b S_{i}-\sum_{j \neq i} \alpha_{j} \sum_{l=1}^{L} \rho_{l} w_{l}=\left(1-m_{i}\right) S_{i}-Q C_{i} \\
& -m_{i} \sum_{j \neq i}\left(\psi_{j}+\alpha_{j} p+\beta_{j} Q C_{j}+\sum_{l=1}^{L} \delta_{j l} w_{l}\right) \\
& \left(1+m_{i}\right) \sum_{j \neq i} \alpha_{j} p-\sum_{j \neq i} \alpha_{j} a-\sum_{j \neq i} \alpha_{j} \sum_{l=1}^{L} \rho_{l} w_{l}=\left(1-m_{i}+\sum_{j \neq i} \alpha_{j} b\right) S_{i}-Q C_{i}
\end{aligned}
$$




$$
\begin{gathered}
-m_{i} \sum_{j \neq i}\left(\psi_{j}+\beta_{j} Q C_{j}+\sum_{l=1}^{L} \delta_{j l} w_{l}\right) \\
\left(1-m_{i}+\sum_{j \neq i} \alpha_{j} b\right) S_{i}=m_{i} \sum_{j \neq i}\left(\psi_{j}+\beta_{j} Q C_{j}\right)-\sum_{j \neq i} \alpha_{j} a+\left(1+m_{i}\right) \sum_{j \neq i} \alpha_{j} p \\
+Q C_{i}+\left(m_{i} \sum_{j \neq i} \sum_{l=1}^{L} \delta_{j l}-\sum_{j \neq i} \sum_{l=1}^{L} \alpha_{j} \rho_{l}\right) w_{l} \\
S_{i}=\frac{m_{i} \sum_{j \neq i} \psi_{j}+m_{i} \sum_{j \neq i} \beta_{j} Q C_{j}-\sum_{j \neq i} \alpha_{j} a}{1-m_{i}+\sum_{j \neq i} \alpha_{j} b}+\frac{\left(1+m_{i}\right) \sum_{j \neq i} \alpha_{j}}{1-m_{i}+\sum_{j \neq i} \alpha_{j} b} p \\
+\frac{m_{i} \sum_{j \neq i} \sum_{l=1}^{L} \delta_{j l}-\sum_{j \neq i} \sum_{l=1}^{L} \alpha_{j} \rho_{l}}{1-m_{i}+\sum_{j \neq i} \alpha_{j} b} w_{l} Q C_{i}+\frac{\sum_{j \neq i} \alpha_{j} b}{1-\beta_{i} Q C_{i}+\sum_{l=1}^{L} \delta_{i l} w_{l} .} \\
\psi_{i}+\alpha_{i} p+\beta_{i}
\end{gathered}
$$

We have the following systems:

$$
\begin{aligned}
\psi_{i} & =\frac{m_{i} \sum_{j \neq i} \psi_{j}+m_{i} \sum_{j \neq i} \beta_{j} Q C_{j}-\sum_{j \neq i} \alpha_{j} a}{1-m_{i}+\sum_{j \neq i} \alpha_{j} b} \\
\alpha_{i} & =\frac{\left(1+m_{i}\right) \sum_{j \neq i} \alpha_{j}}{1-m_{i}+\sum_{j \neq i} \alpha_{j} b} \\
\beta_{i} & =\frac{1}{1-m_{i}+\sum_{j \neq i} \alpha_{j} b} \\
\delta_{i} & =\frac{m_{i} \sum_{j \neq i} \sum_{l=1}^{L} \delta_{j l}-\sum_{j \neq i} \sum_{l=1}^{L} \alpha_{j} \rho_{l}}{1-m_{i}+\sum_{j \neq i} \alpha_{j} b},
\end{aligned}
$$

which can be solved numerically. With $\alpha_{i}$ we solve $\beta_{i}$ for all $i=1,2, \ldots, K$. Then we solve the linear system of $K$ equations for $\psi_{i}$ and the $L$ linear systems of $K$ equations for $\delta$ s. 


\section{Appendix C}

\section{Chapters 4 and 5}

\section{C.1 Proof of corollary 4.2.2}

Consider $a \geq 0, b \geq 0, \rho \geq 0, \mu \geq 0, \psi \geq 0$ and $f(T) \geq 0 \forall T$.

We have:

$$
\begin{aligned}
& \frac{\partial P C\left(p_{t}, T\right)}{\partial a}=1 \geq 0 \\
& \frac{\partial P C\left(p_{t}, T\right)}{\partial x_{1 t}}=\frac{b(K-1-M)}{K(K-2)} e^{-\frac{b(K-1-M)}{K(K-2)} \psi(T-t)} \geq 0 \\
& \frac{\partial P C\left(p_{t}, T\right)}{\partial w_{2}}=\rho \geq 0 \\
& \frac{\partial P C\left(p_{t}, T\right)}{\partial \mu}=\rho(T-t) \geq 0 \\
& \frac{\partial P C\left(p_{t}, T\right)}{\partial \sigma_{1}}=-\left(1-e^{-\frac{b(K-1-M)}{K(K-2)} \psi(T-t)}\right) \frac{\lambda_{1}}{\psi} \leq 0 \\
& \frac{\partial P C\left(p_{t}, T\right)}{\partial \lambda_{1}}=-\left(1-e^{-\frac{b(K-1-M)}{K(K-2)} \psi(T-t)}\right) \frac{\sigma_{1}}{\psi} \leq 0 \\
& \frac{\partial P C\left(p_{t}, T\right)}{\partial \sigma_{2}}=-\rho \lambda_{2}(T-t) \leq 0 \\
& \frac{\partial P C\left(p_{t}, T\right)}{\partial \lambda_{2}}=-\rho \sigma_{2}(T-t) \leq 0 .
\end{aligned}
$$




\section{C.2 Proof of lemma 5.2.1}

The profit function is equal to:

$$
\pi_{i}=p^{c} S_{i}^{*}\left(p^{c}\right)-C\left(S^{*}\left(p^{c}\right)\right)+\left(P C-p^{c}\right) Q C_{i}+m_{i}\left(p^{R}-p^{c}\right) D
$$

The optimal supply function is equal to:

$$
\begin{aligned}
S_{i t}\left(p_{t}, Q C_{i t}, \vec{W}_{t}\right)= & -\frac{a(K-2)}{b(K-1)}+\frac{K-2}{b(K-1)} p_{t}+\frac{1}{K-1} Q C_{i t} \\
& +\frac{m_{i}}{K-1} D_{t}-\frac{(K-2)}{b(K-1)} \sum_{j=1}^{L} \rho_{j} w_{j t} .
\end{aligned}
$$

Now, consider $\overline{Q C}_{j t}=\frac{\sum_{j \neq i} Q C_{j t}}{K-1}$ and $\bar{m}_{j}=\frac{\sum_{j \neq i} m_{j}}{K-1}$. The clearing price $p_{t}^{c}$ is described by the equation:

$$
\begin{aligned}
p_{t}^{c}= & a+\sum_{j=1}^{L} \rho_{j} w_{j t}+b \frac{\left(K-\left(1+m_{i}+(K-1) \bar{m}_{j}\right)\right)}{K(K-2)} D_{t} \\
& -\frac{b}{K(K-2)}\left(Q C_{i}+(K-1) \overline{Q C}_{j}\right) .
\end{aligned}
$$

Substituting back in the firm $i$ 's supply equation, we have the following optimal equilibrium supply:

$$
\begin{aligned}
& S_{i t}\left(p_{t}, Q C_{i t}, \vec{W}_{t}\right) \\
= & \frac{K-2}{b(K-1)}\left(a+\sum_{j=1}^{L} \rho_{j} w_{j t}+b \frac{\left(K-\left(1+m_{i}+(K-1) \bar{m}_{j}\right)\right)}{K(K-2)} D_{t}\right) \\
& -\frac{1}{K(K-1)}\left(Q C_{i}+(K-1) \overline{Q C}_{j}\right)-\frac{a(K-2)}{b(K-1)}+\frac{1}{K-1} Q C_{i t} \\
& +\frac{m_{i}}{K-1} D_{t}-\frac{(K-2)}{b(K-1)} \sum_{j=1}^{L} \rho_{j} w_{j t}
\end{aligned}
$$




$$
\begin{aligned}
&= \frac{K-1-m_{i}-(K-1) \bar{m}_{j}}{K(K-1)} D_{t}-\frac{Q C_{i}+(K-1) \overline{Q C}_{j}}{K(K-1)}+\frac{1}{K-1} Q C_{i t} \\
&+\frac{m_{i}}{K-1} D_{t} \\
&= \frac{K-1-(1-K) m_{i}-(K-1) \bar{m}_{j}}{K(K-1)} D_{t}-\frac{(1-K) Q C_{i}+(K-1) \overline{Q C}_{j}}{K(K-1)} \\
&= \frac{1+m_{i}-\bar{m}_{j}}{K} D_{t}+\frac{Q C_{i}-\overline{Q C}_{j}}{K} \\
& S_{i t}^{*}=\frac{\left(1+m_{i}-\bar{m}_{j}\right) D_{t}}{K}+\frac{Q C_{i t}-\overline{Q C}_{j t}}{K} .
\end{aligned}
$$

Now suppose that the generator/gentailer $i$ has the option to establish/expand his retail position by investing $I$ to increase his retail market share by $\Delta m_{i}$.

Using Taylor expansion, we know that the impact of a change in $m_{i}$ on the gentailer/generator profit is equal to:

$$
\begin{aligned}
\Delta \pi_{i} & =\frac{d \pi_{i}}{d m_{i}} \Delta m_{i}+\frac{1}{2} \frac{d^{2} \pi_{i}}{d m_{i}^{2}}\left(\Delta m_{i}\right)^{2} \\
\Delta \pi_{i} & =\frac{d \pi_{i}}{d \bar{m}_{j}} \Delta \bar{m}_{j}+\frac{1}{2} \frac{d^{2} \pi_{i}}{d \bar{m}_{j}^{2}}\left(\Delta \bar{m}_{j}\right)^{2} .
\end{aligned}
$$

Calculating the derivatives of the first equation and skipping the time subscripts, we have:

$$
\begin{aligned}
\frac{d \pi_{i}\left(p^{c}\left(m_{i}\right), S^{*}\left(m_{i}\right)\right)}{d m_{i}} & =\frac{\partial \pi_{i}}{\partial m_{i}}+\frac{\partial \pi_{i}}{\partial p^{c}} \frac{\partial p^{c}}{\partial m_{i}}+\frac{\partial \pi_{i}}{\partial S_{i}^{*}} \frac{\partial S_{i}^{*}}{\partial m_{i}} \\
\frac{\partial \pi_{i}}{\partial m_{i}} & =\left(p^{R}-p^{c}\right) D \\
\frac{\partial \pi_{i}}{\partial p^{c}} \frac{\partial p^{c}}{\partial m_{i}} & =-\frac{b\left(S-Q C_{i}-m_{i} D\right) D}{K(K-2)}
\end{aligned}
$$




$$
\frac{\partial \pi_{i}}{\partial S_{i}^{*}} \frac{\partial S_{i}^{*}}{\partial m_{i}}=(P-M C) \frac{D}{K} .
$$

Define $M=\sum_{k=1}^{K} m_{k}$ and $\sum_{k=1}^{K} Q C_{k t}=Q C$. Now we show that the first two terms are equal to zero:

$$
\begin{aligned}
& p^{c}-M C_{i}= p^{c}-\left(a+\sum_{j=1}^{L} \rho_{j} w_{j}\right)-b S_{i} \\
&= b \frac{\left.\left(K-1-m_{i}-(K-1) \bar{m}_{j}\right)\right)}{K(K-2)} D-\frac{b\left(Q C_{i}+(K-1) \overline{Q C}_{j}\right)}{K(K-2)} \\
&-b\left(\frac{\left(1+m_{i}-\bar{m}_{j}\right) D}{K}+\frac{Q C_{i t}-\overline{Q C}_{j}}{K}\right) \\
&= \frac{b}{K(K-2)}\left(K-1-m_{i}-(K-1) \bar{m}_{j}\right) D \\
&-\frac{b}{K(K-2)}(K-2)\left(1+m_{i}-\bar{m}_{j}\right) D \\
&-\frac{b\left(Q C_{i}+(K-2) Q C_{i}\right)}{K(K-2)}-\frac{b\left((K-1) \overline{Q C}_{j}-(K-2) \overline{Q C}_{j}\right)}{K(K-2)} \\
&= \frac{b\left(1-(K-1) m_{i}-\bar{m}_{j}\right)}{K(K-2)} D-\frac{b\left((K-1) Q C_{i}+\overline{Q C}_{j}\right)}{K(K-2)} \\
& S_{i}-Q C_{i}-m_{i} D=\frac{\left(1+m_{i}-\bar{m}_{j}\right) D}{K}+\frac{Q C_{i}-\overline{Q C}_{j}}{K}-Q C_{i}-m_{i} D \\
&=\frac{\left(1-(K-1) m_{i}-\bar{m}_{j}\right)}{K} D-\frac{\left((K-1) Q C_{i}+\overline{Q C}_{j}\right)}{K} .
\end{aligned}
$$

Calculating the first order derivative we have:

$$
\frac{\partial \pi_{i}}{\partial p^{c}} \frac{\partial p^{c}}{\partial m_{i}}+\frac{\partial \pi_{i}}{\partial S_{i}^{*}} \frac{\partial S_{i}^{*}}{\partial m_{i}}=0
$$


We also have the following first order derivative:

$$
\frac{d \pi_{i}\left(m_{i}, p^{c}\left(m_{i}\right), S^{*}\left(m_{i}\right)\right)}{d m_{i}}=\left(p^{R}-p^{c}\right) D
$$

The second order derivative is equal to:

$$
\begin{aligned}
\frac{d^{2} \pi_{i}}{d m_{i}^{2}} & =-D \frac{\partial p^{c}}{\partial m_{i}} \\
& =\frac{b}{K(K-2)} D^{2}
\end{aligned}
$$

so that

$$
\Delta \pi_{i t}=\left(p^{R}-p^{c}\right) D \Delta m_{i}+\frac{1}{2} \frac{b}{K(K-2)} D^{2}\left(\Delta m_{i}\right)^{2}
$$

We have an analogous calculation for the impact of a decrease in rival gentailers' average $\bar{m}_{j}$.

Calculating the derivatives of the first equation and skipping the time subscripts, we have:

$$
\begin{aligned}
\frac{d \pi_{i}\left(\bar{m}_{j}, p^{c}\left(\bar{m}_{j}\right), S^{*}\left(\bar{m}_{j}\right)\right)}{d \bar{m}_{j}} & =\frac{\partial \pi_{i}}{\partial \bar{m}_{j}}+\frac{\partial \pi_{i}}{\partial p^{c}} \frac{\partial p^{c}}{\partial \bar{m}_{j}}+\frac{\partial \pi_{i}}{\partial S_{i}^{*}} \frac{\partial S_{i}^{*}}{\partial \bar{m}_{j}} \\
\frac{\partial \pi_{i}}{\partial \bar{m}_{j}} & =0 \\
\frac{\partial \pi_{i}}{\partial p^{c}} \frac{\partial p^{c}}{\partial \bar{m}_{j}} & =(K-1) \frac{\partial \pi_{i}}{\partial p^{c}} \frac{\partial p^{c}}{\partial m_{i}} \\
\frac{\partial \pi_{i}}{\partial S_{i}^{*}} \frac{\partial S_{i}^{*}}{\partial \bar{m}_{j}} & =-\frac{\partial \pi_{i}}{\partial S_{i}^{*}} \frac{\partial S_{i}^{*}}{\partial m_{i}} .
\end{aligned}
$$

Considering that $\frac{\partial \pi_{i}}{\partial p^{c}} \frac{\partial p^{c}}{\partial m_{i}}=-\frac{\partial \pi_{i}}{\partial S_{i}^{*}} \frac{\partial S_{i}^{*}}{\partial m_{i}}$,

we have:

$$
\frac{d \pi_{i}\left(\bar{m}_{j}, p^{c}\left(\bar{m}_{j}\right), S^{*}\left(\bar{m}_{j}\right)\right)}{d \bar{m}_{j}}=K \frac{\partial \pi_{i}}{\partial p^{c}} \frac{\partial p^{c}}{\partial m_{i}}
$$




$$
\begin{aligned}
= & \frac{b\left((K-1) Q C_{i}+\overline{Q C}_{j}\right)}{K(K-2)} D \\
& -\frac{b\left(1-(K-1) m_{i}-\bar{m}_{j}\right)}{K(K-2)} D^{2} .
\end{aligned}
$$

The second order effect is equal to:

$$
\frac{d^{2} \pi_{i}\left(\bar{m}_{j}, p^{c}\left(\bar{m}_{j}\right), S^{*}\left(\bar{m}_{j}\right)\right)}{d \bar{m}_{j}^{2}}=\frac{b}{K(K-2)} D^{2} .
$$

Therefore, we have:

$$
\begin{aligned}
\Delta \pi_{i}= & \frac{b\left((K-1) Q C_{i}+\overline{Q C}_{j}\right)}{K(K-2)} D \Delta \bar{m}_{j}-\frac{b\left(1-(K-1) m_{i}-\bar{m}_{j}\right)}{K(K-2)} D^{2} \Delta \bar{m}_{j} \\
& +\frac{1}{2} \frac{b}{K(K-2)} D^{2}\left(\Delta \bar{m}_{j}\right)^{2} .
\end{aligned}
$$

For simplicity, consider that $\sum_{k=1}^{K} Q C_{k t}$ is a constant $Q C$ and that costs are not driven by any additional state variable. That is, $M C_{i}=a+b S_{i}$ and the clearing price is defined as:

$$
p_{t}^{c}=a+b \frac{(K-1-M)}{K(K-2)} D_{t} \cdot-\frac{b}{K(K-2)} Q C
$$

Define $\Delta \bar{m}_{j}=-\frac{\omega \Delta m_{i}}{K-1}$. Putting the direct $\left(m_{i}\right)$ and indirect $\left(\bar{m}_{j}\right)$ effects together, the investment has the following impact on profit:

$$
\begin{aligned}
\Delta \pi_{i t}= & \Delta m_{i}\left(p^{R}-p^{c}\right) D+\Delta \bar{m}_{j} \frac{b\left((K-1) Q C_{i}+\overline{Q C}_{j}\right)}{K(K-2)} D \\
& +\frac{\left(\Delta m_{i}\right)^{2}}{2} \frac{b}{K(K-2)} D^{2}-\Delta \bar{m}_{j} \frac{b\left(1-(K-1) m_{i}-\bar{m}_{j}\right)}{K(K-2)} D^{2} \\
& +\frac{\left(\Delta \bar{m}_{j}\right)^{2}}{2} \frac{b}{K(K-2)} D^{2} \\
= & \Delta m_{i}\left(p^{R}-a+\frac{b}{K(K-2)} Q C\right) D
\end{aligned}
$$




$$
\begin{aligned}
& +\Delta \bar{m}_{j} \frac{b\left((K-1) Q C_{i}+\overline{Q C}_{j}\right)}{K(K-2)} D \\
& -\Delta m_{i} b \frac{(K-1-M)}{K(K-2)} D^{2}+\frac{\left(\Delta m_{i}\right)^{2}}{2} \frac{b}{K(K-2)} D^{2} \\
& -\Delta \bar{m}_{j} \frac{b\left(1-(K-1) m_{i}-\bar{m}_{j}\right)}{K(K-2)} D^{2}+\frac{\left(\Delta \bar{m}_{j}\right)^{2}}{2} \frac{b}{K(K-2)} D^{2} \\
& =\Delta m_{i}\left(p^{R}-a+\frac{b}{K(K-2)} Q C\right) D \\
& -\frac{\omega \Delta m_{i}}{K-1} \frac{b\left((K-1) Q C_{i}+\overline{Q C}_{j}\right)}{K(K-2)} D \\
& -\Delta m_{i} b \frac{(K-1-M)}{K(K-2)} D^{2}+\frac{\left(\Delta m_{i}\right)^{2}}{2} \frac{b}{K(K-2)} D^{2} \\
& +\frac{\omega \Delta m_{i}}{K-1} \frac{b\left(1-(K-1) m_{i}-\bar{m}_{j}\right)}{K(K-2)} D^{2}+\frac{\omega^{2}\left(\Delta m_{i}\right)^{2}}{2(K-1)^{2}} \frac{b}{K(K-2)} D^{2} \\
& =\Delta m_{i}\left(p^{R}-a+\frac{b}{K(K-2)} Q C-\frac{b \omega\left((K-1) Q C_{i}+\overline{Q C}_{j}\right)}{K(K-1)(K-2)}\right) D \\
& -\frac{b \Delta m_{i}}{K(K-2)}\left(K-1-M-\frac{\Delta m_{i}}{2}\right) D^{2} \\
& +\frac{\omega b \Delta m_{i}}{K(K-1)(K-2)}\left(1-(K-1) m_{i}-\bar{m}_{j}+\frac{\omega \Delta m_{i}}{2(K-1)}\right) D^{2} \\
& =\Delta m_{i}\left(p^{R}-a+\frac{b}{K(K-2)} Q C-\frac{\left.b \omega\left(Q C+K(K-2) Q C_{i}\right)\right)}{K(K-1)^{2}(K-2)}\right) D \\
& -\frac{b \Delta m_{i}}{K(K-2)}\left(K-1-M-\frac{\Delta m_{i}}{2}\right) D^{2} \\
& +\frac{\omega b \Delta m_{i}}{K(K-1)^{2}(K-2)}\left(K-1-M-K(K-2) m_{i}+\frac{\omega \Delta m_{i}}{2}\right) D^{2} \\
& =\Delta m_{i}\left(p^{R}-a+\frac{b}{K(K-2)}\left(1-\frac{\omega}{(K-1)^{2}}\right) Q C-\frac{b \omega}{(K-1)^{2}} Q C_{i}\right) D \\
& -\frac{b \Delta m_{i}}{K(K-2)}\left((K-1-M)\left(1-\frac{\omega}{(K-1)^{2}}\right)\right) D^{2} \\
& -\frac{b \Delta m_{i}}{K(K-2)}\left(\frac{\omega K(K-2)}{(K-1)^{2}} m_{i}-\left(1+\frac{\omega}{(K-1)^{2}}\right) \frac{\Delta m_{i}}{2}\right) D^{2} \\
& =\hat{A} D-\hat{B} D^{2} \text {. }
\end{aligned}
$$




\section{C.3 Proof of lemma 5.2.2}

Consider that the demand follows a geometric brownian motion,

$$
d D_{t}=D_{t} \mu d t+D_{t} \sigma d W_{t}
$$

The present value of the inflows $V_{t}$ is equal to:

$$
\begin{aligned}
V_{t} & =\hat{A} \int_{t}^{T} E_{t}\left[D_{\tau}\right] e^{-r(\tau-t)} d \tau-\hat{B} \int_{t}^{T} E_{t}\left[D_{\tau}^{2}\right] e^{-r(\tau-t)} d \tau \\
& =\hat{A} \int_{t}^{T} e^{\mu(\tau-t)} e^{-r(\tau-t)} d \tau D_{t}-\hat{B} \int_{t}^{T} e^{\left(2 \mu+\sigma^{2}\right)(\tau-t)} e^{-r(\tau-t)} d \tau D_{t}^{2} \\
& =\hat{A} \frac{\left(1-e^{-(r-\mu)(T-t)}\right)}{r-\mu} D_{t}-\hat{B} \frac{\left(1-e^{-\left(r-2 \mu-\sigma^{2}\right)(T-t)}\right)}{r-2 \mu-\sigma^{2}} D_{t}^{2} \\
& =\tilde{A} D_{t}-\tilde{B} D_{t}^{2}
\end{aligned}
$$

Therefore, we have:

$$
V_{t}=\tilde{A} D_{t}-\tilde{B} D_{t}^{2}-I
$$

$\tilde{A}$ is clearly greater than zero if $p^{R}>p^{c}$. We can easily show that $\tilde{B} \geq 0$ as well. By contradiction, suppose that $\tilde{B}<0$. Then the following holds:

$$
\begin{aligned}
\left(1+\frac{\omega}{(K-1)^{2}}\right) \frac{\Delta m_{i}}{2} & \geq(K-1-M)\left(1-\frac{\omega}{(K-1)^{2}}\right)+\frac{\omega K(K-2)}{(K-1)^{2}} m_{i} \\
\left(1+\frac{\omega}{(K-1)^{2}}\right) \frac{\Delta m_{i}}{2} & \geq(K-1-M)\left(1-\frac{\omega}{(K-1)^{2}}\right) \\
\Delta m_{i} & \geq 2 \frac{1-\frac{\omega}{(K-1)^{2}}}{1+\frac{\omega}{(K-1)^{2}}}(K-1-M) .
\end{aligned}
$$


The second term of the inequality achieves his minimum value if $\omega=1$, $K=3$ and $M=1$. Therefore in this case,

$$
\begin{aligned}
\Delta m_{i} & \geq 2 \frac{1-\frac{1}{(3-1)^{2}}}{1+\frac{1}{(3-1)^{2}}}(3-1-1) \\
\Delta m_{i} & \geq \frac{6}{5}
\end{aligned}
$$

However, this is a contradiction because $\Delta m_{i} \leq 1$. Therefore, $\tilde{B} \geq 0$.

\section{C.4 Proof of proposition 5.2.3}

Over a short period of time, $d t$, the total return from holding the investment opportunity, $F(D)$, is simply its expected change in value. With a rate of return $r$, this is:

$$
r F d t=E(d F)
$$

Expanding $d F$ using Ito's Lemma, we get the quadratic differential equation:

$$
\frac{1}{2} \sigma^{2} D^{2} F^{\prime \prime}(D)+\mu D F^{\prime}(D)-r F=0
$$

The general solutions for $F$ in the domains $\left[0, D_{1}^{*}[\right.$ and $] D_{2}^{*}, \infty[$ are equal to $F=\alpha_{11} D^{\beta_{1}}+\alpha_{21} D^{\beta_{2}}$ and $F=\alpha_{21} D^{\beta_{1}}+\alpha_{22} D^{\beta_{2}}$, respectively. The parameters $\beta_{1}$ and $\beta_{2}$ are the characteristic roots of the partial differential equation given by equation (5.4). We know that $\beta_{1}$ is greater than 1 and $\beta_{2}$ is negative. ${ }^{1}$ Therefore, the conditions given by equations (5.5) and (5.10) imply that $\alpha_{21}=0$ and $\alpha_{12}=0$. We obtain $\alpha_{11}$ and $\alpha_{22}$ from the remaining boundary conditions.

Assume that, for $D \in\left[0, D_{1}^{*}\right], F(D)$ satisfies the following boundary

\footnotetext{
${ }^{1}$ See Dixit et al. (1994) pages 142-144.
} 
conditions:

$$
\begin{aligned}
F(0) & =0 \\
F\left(D_{1}^{*}\right) & =\tilde{A} D_{1}^{*}-\tilde{B} D_{1}^{* 2}-I \\
F^{\prime}\left(D_{1}^{*}\right) & =\tilde{A}-2 \tilde{B} D_{1}^{*} .
\end{aligned}
$$

Using the first boundary condition we have $F(D)=\alpha_{11} D^{\beta_{1}}$ where:

$$
\beta_{1}=\frac{1}{2}-\frac{(r-\mu)}{\sigma^{2}}+\sqrt{\left(\frac{(r-\mu)}{\sigma^{2}}-\frac{1}{2}\right)^{2}+\frac{2 r}{\sigma^{2}}} .
$$

Applying the boundary conditions we have:

$$
\begin{aligned}
\alpha_{11} \beta_{1} D_{1}^{* \beta_{1}-1} & =\tilde{A}-2 \tilde{B} D_{1}^{*} \\
\alpha_{11} D_{1}^{* \beta_{1}} & =\tilde{A} D_{1}^{*}-\tilde{B} D_{1}^{* 2}-I .
\end{aligned}
$$

Dividing C.20 by C.21,

$$
\begin{aligned}
\frac{1}{\beta_{1}} D_{1}^{*} & =\frac{\tilde{A} D_{1}^{*}-\tilde{B} D_{1}^{* 2}-I}{\tilde{A}-2 \tilde{B} D_{1}^{*}} \\
& \Rightarrow \frac{\tilde{A}}{\beta} D_{1}^{*}-2 \frac{\tilde{B}}{\beta} D_{1}^{* 2}=\tilde{A} D_{1}^{*}-\tilde{B} D_{1}^{* 2}-I \\
& \Rightarrow-\frac{2-\beta_{1}}{\beta_{1}} \tilde{B} D_{1}^{* 2}+\frac{1-\beta_{1}}{\beta_{1}} \tilde{A} D_{1}^{*}+I=0 \\
& \Rightarrow-\left(2-\beta_{1}\right) \tilde{B} D_{1}^{* 2}+\left(1-\beta_{1}\right) \tilde{A} D_{1}^{*}+\beta_{1} I=0 .
\end{aligned}
$$

Lastly, we have:

$$
\begin{aligned}
D_{1}^{*} & =\frac{\left(1-\beta_{1}\right) \tilde{A}+\sqrt{\left(1-\beta_{1}\right)^{2} \tilde{A}^{2}+4\left(2-\beta_{1}\right) \beta_{1} \tilde{B} I}}{2\left(2-\beta_{1}\right) \tilde{B}} \\
\alpha_{11} & =\frac{\tilde{A}}{\beta_{1}} D_{1}^{* 1-\beta_{1}}-2 \frac{\tilde{B}}{\beta_{1}} D_{1}^{* 2-\beta_{1}} .
\end{aligned}
$$


It is reasonable to assume that if the project has a negative value at a the critical demand, the option to exercise it has no value.

Therefore, $\alpha_{11}$ is, in fact, equal to:

$$
\alpha_{11}=\max \left(\frac{\tilde{A}}{\beta} D^{* 1-\beta}-2 \frac{\tilde{B}}{\beta} D^{* 2-\beta}, 0\right) .
$$

For $D \in\left[D_{2}^{*}, \infty\right]$ we have the following boundary conditions:

$$
\begin{aligned}
F(\infty) & =0 \\
F\left(D_{2}^{*}\right) & =\tilde{A} D_{2}^{*}-\tilde{B} D_{2}^{* 2}-I \\
F^{\prime}\left(D_{2}^{*}\right) & =\tilde{A}-2 \tilde{B} D_{2}^{*}
\end{aligned}
$$

Using the transversality condition, the solution in this region is $F(D)=$ $\alpha_{22} D^{\beta_{2}}$, where:

$$
\beta_{2}=\frac{1}{2}-\frac{(r-\mu)}{\sigma^{2}}-\sqrt{\left(\frac{(r-\mu)}{\sigma^{2}}-\frac{1}{2}\right)^{2}+\frac{2 r}{\sigma^{2}}}
$$

Analogously, we have the following results:

$$
\begin{aligned}
D_{2}^{*} & =\frac{\left(1-\beta_{2}\right) \tilde{A}+\sqrt{\left(1-\beta_{2}\right)^{2} \tilde{A}^{2}+4\left(2-\beta_{2}\right) \beta_{2} \tilde{B} I}}{2\left(2-\beta_{2}\right) \tilde{B}} \\
\alpha_{22} & =\frac{\tilde{A}}{\beta_{2}} D_{2}^{* 1-\beta_{2}}-2 \frac{\tilde{B}}{\beta_{2}} D_{2}^{* 2-\beta_{2}} .
\end{aligned}
$$




\section{Appendix D}

\section{Chapter 6}

\section{D.1 Proof of proposition 6.3.1}

Assuming that the objective function is twice continuously differentiable, to solve: $\max _{Q C_{i}} F\left(\pi_{i}^{* G}\left(Q C_{i}, p^{c}\left(Q C_{i}\right), S\left(Q C_{i}\right)\right)\right)=E\left[\pi_{i}^{* G}\right]+\frac{\lambda_{G}}{2} \operatorname{Var}\left[\pi_{i}^{* G}\right]$, we need first to calculate the first order condition. Skipping subscripts and superscripts, we know that:

$$
\frac{d F\left(Q C, p^{c}(Q C), S(Q C)\right)}{d Q C}=\frac{\partial F}{Q C}+\frac{\partial F}{\partial p^{c}} \frac{\partial p^{c}(Q C)}{\partial Q C}+\frac{\partial F}{\partial S} \frac{\partial S(Q C)}{\partial Q C}=0 .
$$

Solving the differential we have:

$$
\begin{aligned}
\frac{d F(Q C, p(Q C), S(Q C))}{d Q C}= & P C-E(p)-\lambda_{G} \sigma^{2} Q C+\lambda_{G} \operatorname{cov}\left(p, \Upsilon^{*}\right) \\
& +\frac{\partial F}{\partial p} \frac{\partial p}{\partial Q C}+\frac{\partial F(S)}{\partial S} \frac{\partial S(Q C)}{\partial Q C}=0 .
\end{aligned}
$$

Now, assume $B=\frac{b}{N(N-2)}$. Solving for $\frac{\partial F}{\partial p} \frac{\partial p}{\partial Q C}$ and $\frac{\partial F(S)}{\partial S} \frac{\partial S(Q C)}{\partial Q C}$, the following applies:

$$
\frac{\partial F}{\partial p^{c}} \frac{\partial p^{c}}{\partial Q C}=E\left(\frac{\partial \pi}{\partial p^{c}} \frac{\partial p^{c}}{\partial Q C}\right)-\frac{\lambda_{G}}{2}\left(2 E\left(\pi \frac{\partial \pi}{\partial p^{c}} \frac{\partial p^{c}}{\partial Q C}\right)-2 E(\pi) E\left(\frac{\partial \pi}{\partial p^{c}} \frac{\partial p^{c}}{\partial Q C}\right)\right)
$$




$$
\begin{aligned}
& =E\left(\frac{\partial \pi}{\partial p^{c}} \frac{\partial p^{c}}{\partial Q C}\right)-\lambda_{G} \operatorname{cov}\left(\pi, \frac{\partial \pi}{\partial p^{c}} \frac{\partial p^{c}}{\partial Q C}\right) \\
& =-B\left(E\left(S^{*}-Q C\right)-\lambda_{G} \operatorname{cov}\left(\pi, S^{*}\right)\right) \\
\frac{\partial F}{\partial S} \frac{\partial S}{\partial Q C} & =E\left(\frac{\partial \pi}{\partial S} \frac{\partial S}{\partial Q C}\right)-\frac{\lambda_{G}}{2}\left(2 E\left(\pi \frac{\partial \pi}{\partial S} \frac{\partial S}{\partial Q C}\right)-2 E(\pi) E\left(\frac{\partial \pi}{\partial S} \frac{\partial S}{\partial Q C}\right)\right) \\
& =E\left(\frac{\partial \pi}{\partial S} \frac{\partial S}{\partial Q C}\right)-\lambda_{G} \operatorname{cov}\left(\pi, \frac{\partial \pi}{\partial S} \frac{\partial S}{\partial Q C}\right) \\
& =\frac{1}{K}\left(E\left(p^{c}-M C\right)-\lambda_{G} \operatorname{cov}\left(\pi, p^{c}-M C\right)\right) .
\end{aligned}
$$

From equation (6.8) we have $\frac{\tilde{D}}{K}=S^{*}$. Subtracting $M C_{i}=a+b S_{i}+$ $\sum_{j=1}^{L} \rho_{j} w_{j}$ from equation (6.9), we have:

$$
\begin{aligned}
p^{c}-M C_{i} & =-b S^{*}-\frac{b}{K-2} Q C^{* G}+\frac{b(K-1)}{K(K-2)} \tilde{D} \\
& =\frac{b(K-1)}{K-2} \frac{\tilde{D}}{K}-b \frac{\tilde{D}}{K}-\frac{b}{K-2} Q C^{* G} \\
& =\frac{b}{K-2}\left(\frac{\tilde{D}}{K}-Q C^{* G}\right) .
\end{aligned}
$$

Last,

$$
\begin{aligned}
\frac{\partial F}{\partial S} \frac{\partial S}{\partial Q C} & =B\left(E\left(\frac{\tilde{D}}{K}-Q C^{* G}\right)-\lambda_{G} \operatorname{cov}\left(\pi, \frac{\tilde{D}}{K}\right)\right) \\
& =B\left(E\left(S^{*}-Q C\right)-\lambda_{G} \operatorname{cov}\left(\pi, S^{*}\right)\right),
\end{aligned}
$$

which means that,

$$
\frac{\partial F}{\partial p^{c}} \frac{\partial p}{\partial Q C}+\frac{\partial F(S)}{\partial S} \frac{\partial S(Q C)}{\partial Q C}=0
$$

Therefore,

$$
Q C^{* G}=\frac{P C-E\left(p^{c}\right)}{\lambda_{G} \sigma^{2}}+\frac{\operatorname{cov}\left(p^{c}, \Upsilon^{g *}\right)}{\sigma^{2}}
$$


Since the quantity contracted by retailers does not have indirect effects on their profits (they do not affect spot prices, demand or retail prices), the solution for the retailers' problem is straightforward and equal to:

$$
Q C_{i}^{* R}=\frac{P C-E\left(p^{c}\right)}{\lambda_{R} \sigma^{2}}+m_{i} \frac{\operatorname{cov}\left(p^{c},\left(p^{R}-p^{c}\right) \tilde{D}\right)}{\sigma^{2}} .
$$

\section{D.2 Proof of proposition 6.3.2}

Clearing the forward market we have:

$$
\begin{aligned}
\sum_{i=1}^{K} Q C_{i}^{* G}= & K Q C^{* G} \\
= & K\left(\frac{P C-E\left(p^{c}\right)}{\lambda_{G} \sigma^{2}}+\frac{\operatorname{cov}\left(p^{c}, \Upsilon\left(S^{*}, p^{c}\right)\right)}{\sigma^{2}}\right) \\
\sum_{i=1}^{R} Q C_{i}^{* R}= & R Q C^{* R} \\
= & R \frac{P C-E\left(p^{c}\right)}{\lambda_{R} \sigma^{2}}+\frac{\operatorname{cov}\left(p^{c},\left(p^{R}-p^{c}\right) \tilde{D}\right)}{\sigma^{2}} \\
\Rightarrow \sigma^{2} \sum_{i=1}^{N} Q C_{i}^{*}= & \left(\frac{K}{\lambda_{G}}+\frac{R}{\lambda_{R}}\right)\left(P C^{*}-E\left(p^{c}\right)\right)+ \\
& K \operatorname{cov}\left(p^{c}, \Upsilon\left(S^{*}, p^{c}\right)\right)+\operatorname{cov}\left(p^{c},\left(p^{R}-p^{c}\right) \tilde{D}\right)=0 \\
\Rightarrow P C^{*}-E\left(p^{c}\right)= & -\frac{K \operatorname{cov}\left(p^{c}, \Upsilon^{*}\right)+\operatorname{cov}\left(p^{c},\left(P^{R}-p^{c}\right) \tilde{D}\right)}{\frac{R}{\lambda_{R}}+\frac{K}{\lambda_{G}}} .
\end{aligned}
$$

Substituting the equation above in the equation (6.10), we have the equation (6.13):

$$
\begin{aligned}
Q C^{* G} & =-\frac{K \operatorname{cov}\left(p^{c}, \Upsilon^{*}\right)+\operatorname{cov}\left(p^{c},\left(P^{R}-p^{c}\right) \tilde{D}\right)}{\lambda_{G} \sigma^{2}\left(\frac{R}{\lambda_{R}}+\frac{K}{\lambda_{G}}\right)}+\frac{\operatorname{cov}\left(p^{c}, \Upsilon^{*}\right)}{\sigma^{2}} \\
& =-\frac{K \operatorname{cov}\left(p^{c}, \Upsilon^{*}\right)+\operatorname{cov}\left(p^{c},\left(P^{R}-p^{c}\right) \tilde{D}\right)}{\sigma^{2}\left(\frac{\lambda_{G}}{\lambda_{R}} R+K\right)}+\frac{\operatorname{cov}\left(p^{c}, \Upsilon^{*}\right)}{\sigma^{2}}
\end{aligned}
$$




$$
\begin{aligned}
& =-\frac{\operatorname{cov}\left(p^{c},\left(P^{R}-p^{c}\right) \tilde{D}\right)}{\sigma^{2}\left(\frac{\lambda_{G}}{\lambda_{R}} R+K\right)}+\frac{1}{\sigma^{2}}\left(1-\frac{K}{\frac{\lambda_{G}}{\lambda_{R}} R+K}\right) \operatorname{cov}\left(p^{c}, \Upsilon^{*}\right) \\
& =-\frac{\operatorname{cov}\left(p^{c},\left(P^{R}-p^{c}\right) \tilde{D}\right)}{\sigma^{2}\left(\frac{\lambda_{G}}{\lambda_{R}} R+K\right)}+\frac{1}{\sigma^{2}}\left(\frac{\frac{\lambda_{G}}{\lambda_{R}} R+K-K}{\left(\frac{\lambda_{G}}{\lambda_{R}} R+K\right)}\right) \operatorname{cov}\left(p^{c}, \Upsilon^{*}\right) \\
& =-\left(\frac{\operatorname{cov}\left(p^{c},\left(P^{R}-p^{c}\right) \tilde{D}\right)}{\sigma^{2}\left(\frac{\lambda_{G}}{\lambda_{R}} R+K\right)}-\frac{1}{\sigma^{2}}\left(\frac{\frac{\lambda_{G}}{\lambda_{R}} R}{\frac{\lambda_{G}}{\lambda_{R}} R+K}\right) \operatorname{cov}\left(p^{c}, \Upsilon^{*}\right)\right) \times \frac{\frac{\lambda_{R}}{\lambda_{G}} \frac{1}{R}}{\frac{\lambda_{R}}{\lambda_{G}} \frac{1}{R}} \\
& =\frac{\operatorname{cov}\left(p^{c}, \Upsilon^{*}\right)-\frac{\lambda_{R}}{\lambda_{G}} \frac{\operatorname{cov}\left(p^{c},\left(P^{R}-p^{c}\right) \tilde{D}\right)}{R}}{\sigma^{2}\left(1+\frac{K}{R} \frac{\lambda_{R}}{\lambda_{G}}\right)} \\
& Q C_{i}^{* R}=-\frac{K \operatorname{cov}\left(p^{c}, \Upsilon^{*}\right)+\operatorname{cov}\left(p^{c},\left(P^{R}-p^{c}\right) \tilde{D}\right)}{\sigma^{2}\left(\frac{\lambda_{G}}{\lambda_{R}} R+K\right)}+m_{i} \frac{\operatorname{cov}\left(p^{c},\left(P^{R}-p^{c}\right) \tilde{D}\right)}{\sigma^{2}} \\
& =-\frac{K \operatorname{cov}\left(p^{c}, \Upsilon^{*}\right)+\left(1-m_{i}\left(\frac{\lambda_{G}}{\lambda_{R}} R+K\right)\right) \operatorname{cov}\left(p^{c},\left(P^{R}-p^{c}\right) \tilde{D}\right)}{\sigma^{2}\left(\frac{\lambda_{G}}{\lambda_{R}} R+K\right)} \\
& =-\frac{\frac{K}{R} \operatorname{cov}\left(p^{c}, \Upsilon^{*}\right)+\left(\frac{1}{R}-m_{i}\left(\frac{\lambda_{R}}{\lambda_{G}}+\frac{K}{R}\right)\right) \operatorname{cov}\left(p^{c},\left(p^{R}-p^{c}\right) \tilde{D}\right)}{\sigma^{2}\left(\frac{\lambda_{R}}{\lambda_{G}}+\frac{K}{R}\right)}
\end{aligned}
$$

\section{D.3 Proof of proposition 6.3.3}

Given $\frac{\tilde{D}}{K}=S^{*}$ in equilibrium and substituting D.2 in D.1, we have:

$$
p^{c}-M C_{i}=\frac{b}{(K-2)}\left(\frac{\tilde{D}}{K}-\frac{\operatorname{cov}\left(p^{c}, \Upsilon^{*}\right)-\frac{\lambda_{R}}{\lambda_{G}} \frac{\operatorname{cov}\left(p^{c},\left(P^{R}-p^{c}\right) \tilde{D}\right)}{R}}{\sigma^{2}\left(1+\frac{K}{R} \frac{\lambda_{R}}{\lambda_{G}}\right)}\right)
$$


which gives the following expectation:

$$
E\left(p^{c}\right)-M C_{i}=\frac{b}{(K-2)}\left(\frac{E(\tilde{D})}{K}-\frac{\left.\operatorname{cov}\left(p^{c}, \Upsilon^{*}\right)-\frac{\lambda_{R}}{\lambda_{G}} \frac{\operatorname{cov}\left(p^{c},\left(P^{R}-p^{c}\right) \tilde{D}\right)}{R}\right)}{\sigma^{2}\left(1+\frac{K}{R} \frac{\lambda_{R}}{\lambda_{G}}\right)}\right)
$$

